JOURNAL OF THE

AMERICAN MATHEMATICAL SOCIETY

Volume 14, Number 1, Pages 25-78

S 0894-0347(00)00348-9

Article electronically published on October 2, 2000

\title{
MATING SIEGEL QUADRATIC POLYNOMIALS
}

\author{
MICHAEL YAMPOLSKY AND SAEED ZAKERI
}

\section{Contents}

1. Introduction

2. Background material

3. The Blaschke model for Petersen's theorem

4. A Blaschke model for mating

5. Construction of puzzle-pieces

6. Complex bounds

7. The proof of the Main Theorem

8. Concluding remarks

References

\section{INTRODUCTION}

1.1. Mating: Definitions and some history. Mating quadratic polynomials is a topological construction suggested by Douady and Hubbard [Do2] to partially parametrize quadratic rational maps of the Riemann sphere by pairs of quadratic polynomials. Some results on matings of higher degree maps exist, but we will not discuss them in this paper. While there exist several, presumably equivalent, ways of describing the construction of mating, the following approach is perhaps the most standard. Consider two monic quadratic polynomials $f_{1}$ and $f_{2}$ whose filled Julia sets $K\left(f_{i}\right)$ are locally-connected. For each $f_{i}$, let $\Phi_{i}$ denote the conformal isomorphism between the basin of infinity $\widehat{\mathbb{C}} \backslash K\left(f_{i}\right)$ and $\widehat{\mathbb{C}} \backslash \overline{\mathbb{D}}$, with $\Phi_{i}(\infty)=\infty$ and $\Phi_{i}^{\prime}(\infty)=1$. These Bötcher maps conjugate the polynomials to the squaring map:

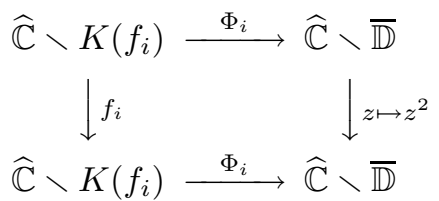

By Carathéodory's Theorem the inverse map $\Phi_{i}^{-1}$ has a continuous extension

$$
\Phi_{i}^{-1}: \partial \mathbb{D} \rightarrow J\left(f_{i}\right)
$$

Received by the editors March 25, 1999 and, in revised form, June 9, 2000.

2000 Mathematics Subject Classification. Primary 37F10; Secondary 37F45, 37F50.

Key words and phrases. Holomorphic dynamics, rational map, Siegel disk, mating, Julia set.

The first author was partially supported by NSF grant DMS-9804606. 
where the Julia set $J\left(f_{i}\right)=\partial K\left(f_{i}\right)$ is the topological boundary of the filled Julia set. The induced parametrization

$$
\gamma_{i}(t)=\Phi_{i}^{-1}\left(e^{2 \pi i t}\right): \mathbb{T}=\mathbb{R} / \mathbb{Z} \rightarrow J\left(f_{i}\right)
$$

is commonly referred to as the Carathéodory loop of $J\left(f_{i}\right)$. Note that by the above commutative diagram, $\gamma_{i}(2 t)=f_{i}\left(\gamma_{i}(t)\right)$. Consider the topological space

$$
X=\left(K\left(f_{1}\right) \sqcup K\left(f_{2}\right)\right) /\left(\gamma_{1}(t) \sim \gamma_{2}(-t)\right)
$$

obtained by gluing the two filled Julia sets along their Carathéodory loops in reverse directions.

Definition I. Assume that the space $X$ as defined above is homeomorphic to the 2 -sphere $S^{2}$. Then the pair of polynomials $\left(f_{1}, f_{2}\right)$ is called topologically mateable. The induced map of $S^{2}$

$$
f_{1} \sqcup_{\mathcal{T}} f_{2}=\left(\left.\left.f_{1}\right|_{K_{1}} \sqcup f_{2}\right|_{K_{2}}\right) /\left(\gamma_{1}(t) \sim \gamma_{2}(-t)\right)
$$

is the topological mating of $f_{1}$ and $f_{2}$.

It may seem surprising at this point that topologically mateable quadratics even exist. However, we shall see below that such examples are abundant. For any mateable pair $\left(f_{1}, f_{2}\right)$, their topological mating is a degree 2 branched covering of the sphere, and it is natural to ask whether it possesses an invariant conformal structure.

Definition II. A quadratic rational map $F: \widehat{\mathbb{C}} \rightarrow \widehat{\mathbb{C}}$ is called a conformal mating, or simply a mating, of $f_{1}$ and $f_{2}$,

$$
F=f_{1} \sqcup f_{2},
$$

if it is conjugate to the topological mating $f_{1} \sqcup_{\mathcal{T}} f_{2}$ by a homeomorphism which is conformal in the interiors of $K\left(f_{1}\right)$ and $K\left(f_{2}\right)$ in case there is an interior. If such $F$ is unique up to conjugation by a Möbius transformation, we refer to it as the mating of $f_{1}$ and $f_{2}$.

Before proceeding to formulate the known existence results, let us describe another equivalent method of defining a mating. Let (c) denote the complex plane $\mathbb{C}$ compactified by adjoining a circle of directions at infinity $\left\{\infty \cdot e^{2 \pi i t} \mid t \in \mathbb{T}\right\}$ with the natural topology. Each $f_{i}$ extends continuously to a copy of $\subset_{i}$, acting as the squaring map $z \mapsto z^{2}$ on the circle at infinity. Gluing the disks (C) ${ }_{i}$ together via the equivalence relation $\sim_{\infty}$ identifying the point $\infty \cdot e^{2 \pi i t} \in \subset_{C_{1}}$ with $\infty \cdot e^{-2 \pi i t} \in \subset_{C_{2}}$, we obtain a 2 -sphere $\left(\left(_{1} \sqcup \bigodot_{2}\right) / \sim_{\infty}\right.$. The well-defined map $f_{1} \sqcup_{\mathcal{F}} f_{2}$ on this sphere given by $f_{i}$ on $\left(_{i}\right.$ is a degree 2 branched covering of the sphere with an invariant equator. We shall refer to this map as the formal mating of $f_{1}, f_{2}$.

Recall that the external ray of $f_{i}$ at angle $t$ is the preimage

$$
R_{i}(t)=\Phi_{i}^{-1}\left(\left\{r e^{2 \pi i t} \mid r>1\right\}\right)
$$

for $t \in \mathbb{T}$. Let $\hat{R}_{i}(t)$ denote the closure of $R_{i}(t)$ in $\left(_{i}\right.$. The ray equivalence relation $\sim_{r}$ on $\left(\subset_{1} \sqcup C_{2}\right) / \sim_{\infty}$ is defined as follows. The points $z$ and $w$ are equivalent, $z \sim_{r} w$, if and only if there exists a collection of closed rays $\hat{R}_{j}=\hat{R}_{i}\left(t_{j}\right)$, $i \in\{1,2\}$ and $j=1, \ldots, n$, such that $z \in \hat{R}_{1}, w \in \hat{R}_{n}$ and $\hat{R}_{j} \cap \hat{R}_{j+1} \neq \emptyset$ for $j=1, \ldots, n-1$. It follows immediately from the definition that if $f_{1}$ and $f_{2}$ are 
topologically mateable, then the quotient of $\left((\mathcal{C})_{1} \sqcup\left(_{2}\right) / \sim_{\infty}\right.$ modulo $\sim_{r}$ is again a 2-sphere, and

$$
\left(f_{1} \sqcup_{\mathcal{F}} f_{2}\right) / \sim_{r} \simeq f_{1} \sqcup_{\mathcal{T}} f_{2} .
$$

Finally, let us formulate another definition of conformal mating, equivalent to the one previously given, but more convenient for further application:

Definition IIa. Let $f_{1}$ and $f_{2}$ be quadratic polynomials with locally-connected Julia sets. A quadratic rational map $F$ of the Riemann sphere is called a conformal mating of $f_{1}$ and $f_{2}$ if there exist continuous semiconjugacies

$$
\varphi_{i}: K\left(f_{i}\right) \rightarrow \widehat{\mathbb{C}}, \text { with } \varphi_{i} \circ f_{i}=F \circ \varphi_{i},
$$

conformal in the interiors of the filled Julia sets in case there is an interior, such that $\varphi_{1}\left(K\left(f_{1}\right)\right) \cup \varphi_{2}\left(K\left(f_{2}\right)\right)=\widehat{\mathbb{C}}$ and for $i, j=1,2, \varphi_{i}(z)=\varphi_{j}(w)$ if and only if $z \sim_{r} w$.

We are now prepared to give an account of known results. The simplest example of a non-mateable pair is given by quadratic polynomials $f_{c_{1}}(z)=z^{2}+c_{1}$ and $f_{c_{2}}(z)=z^{2}+c_{2}$ with locally-connected Julia sets whose parameter values $c_{1}$ and $c_{2}$ belong to a pair of conjugate limbs of the Mandelbrot set. In this case the rays $\left\{R_{1}\left(t_{j}\right)\right\}$ and $\left\{R_{2}\left(t_{j}\right)\right\}$ landing at the dividing fixed points $\alpha_{1}, \alpha_{2}$ of the two polynomials have opposite angles (see e.g. [Mi3]). This implies $\alpha_{1} \sim_{r} \alpha_{2}$, and it is not hard to check that the quotient of $\left(\subset_{1} \sqcup C_{C}\right) / \sim_{\infty}$ modulo $\sim_{r}$ is not homeomorphic to the 2 -sphere.

Recall that two branched coverings $F$ and $G$ of $S^{2}$ with finite postcritical sets $P_{F}$ and $P_{G}$ are equivalent combinatorially or in the sense of Thurston if there exist two orientation-preserving homeomorphisms $\phi, \psi: S^{2} \rightarrow S^{2}$, such that $\phi \circ F=G \circ \psi$, and $\psi$ is isotopic to $\phi$ rel $P_{F}$. Using Thurston's characterization of critically finite rational maps as branched coverings of the sphere (see $[\mathrm{DH}]$ ), Tan Lei [Tan and Rees [Re1] established the following:

Theorem. Let $c_{1}$ and $c_{2}$ be two parameter values not in conjugate limbs of the Mandelbrot set such that $f_{c_{1}}$ and $f_{c_{2}}$ are postcritically finite. Then the map $F$ is combinatorially equivalent to a quadratic rational map, where $F$ is either the formal mating $f_{c_{1}} \sqcup_{\mathcal{F}} f_{c_{2}}$ or a certain degenerate form of it.

Taking this line of investigation further, Rees [Re2] and Shishikura [Sh] demonstrated:

Theorem. Under the assumptions of the previous theorem, $f_{c_{1}}$ and $f_{c_{2}}$ are topologically mateable. Moreover, their conformal mating $f_{c_{1}} \sqcup f_{c_{2}}$ exists.

The case where the critical points of $f_{c_{i}}$ are periodic was considered by Rees, the complementary case was done by Shishikura. Note, in particular, that when none of the critical points is periodic, the Julia sets are dendrites with no interior, which makes the result particularly striking. An example of this phenomenon is analyzed in detail in Milnor's recent paper [Mi4] in which he considers the selfmating $F=f_{c_{1 / 4}} \sqcup f_{c_{1 / 4}}$, where the quadratic polynomial $f_{c_{1 / 4}}$ is the landing point of the 1/4-external ray of the Mandelbrot set. It is not hard to deduce $F$ is a Lattès map whose Julia set $J(F)=\widehat{\mathbb{C}}$ is obtained by pasting together two copies of the dendrite $J\left(f_{c_{1 / 4}}\right)$. 
The issue of topological mateability is usually settled using the following result of R. L. Moore [Mo]. Recall that an equivalence relation $\sim$ on $S^{2}$ is closed if $x_{n} \rightarrow x$, $y_{n} \rightarrow y$ and $x_{n} \sim y_{n}$ implies $x \sim y$.

Theorem (Moore). Suppose that $\sim$ is a closed equivalence relation on the 2-sphere $S^{2}$ such that every equivalence class is a compact connected non-separating proper subset of $S^{2}$. Then the quotient space $S^{2} / \sim$ is again homeomorphic to $S^{2}$.

For the application at hand, the theorem is replaced by the following corollary (see for example Proposition 4.4. of [ST] ):

Corollary. Let $f_{1}$ and $f_{2}$ be two quadratic polynomials with locally-connected Julia sets, such that every class of the ray equivalence relation $\sim_{r}$ is non-separating and contains at most $N$ external rays for a fixed $N>0$. Then $f_{1}$ and $f_{2}$ are topologically mateable.

By means of a standard quasiconformal surgery, the theorem of Rees and Shishikura can be extended to any pair $f_{c_{1}}, f_{c_{2}}$ where the $c_{i}$ belong to hyperbolic components $H_{1}, H_{2}$ of the Mandelbrot set which do not belong to conjugate limbs. Mating thus yields an isomorphism between the product $H_{1} \times H_{2}$ and a hyperbolic component in the parameter space of quadratic rational maps. This isomorphism, however, does not necessarily extend as a continuous map to the product of closures $\bar{H}_{1} \times \bar{H}_{2}$, as was recently shown by A. Epstein Ep.

So far no example of conformal matings without using Thurston's theorem (that is, going beyond the postcritically finite/hyperbolic case) has appeared in the literature. However, J. Luo in his dissertation [Luo has outlined a proof of the existence of conformal matings of Yoccoz polynomials with star-like polynomials (centers of hyperbolic components attached to the main cardioid of the Mandelbrot set). His approach consists of locating a candidate rational map for the mating, and then using Yoccoz puzzle partitions and complex bounds of Yoccoz to prove that this candidate rational map is a mating. A somewhat similar philosophy plays a role in this paper.

The question of constructing matings of polynomials with connected but nonlocally-connected Julia sets has been completely untouched. While there are definitions of mating which would carry over to the non-locally-connected case (such as approximate matings discussed in [Mi2], p. 54), no examples of such matings are known.

1.2. Statement of the results. Consider an irrational number $0<\theta<1$ and the quadratic polynomial $z \mapsto e^{2 \pi i \theta} z+z^{2}$ which has an indifferent fixed point with multiplier $e^{2 \pi i \theta}$ at the origin. To make this polynomial centered, we conjugate it by an affine map of $\mathbb{C}$ to put it in the normal form

$$
f_{\theta}: z \mapsto z^{2}+c_{\theta}, \text { with } c_{\theta}=\frac{e^{2 \pi i \theta}}{2}\left(1-\frac{e^{2 \pi i \theta}}{2}\right) .
$$

The corresponding indifferent fixed point of $f_{\theta}$ is denoted by $\alpha$. Assuming $\theta$ is irrational of bounded type, a classical result of Siegel Si] implies that $f_{\theta}$ is linearizable near $\alpha$, i.e., there exist an open neighborhood $U$ of $\alpha$ and a conformal isomorphism $\phi: U \stackrel{\simeq}{\longrightarrow} \mathbb{D}$ which conjugates $f_{\theta}$ on $U$ to the rigid rotation $\varrho_{\theta}: z \mapsto e^{2 \pi i \theta} z$ by angle $\theta:$

$$
\phi \circ f_{\theta} \circ \phi^{-1}=\varrho_{\theta} .
$$




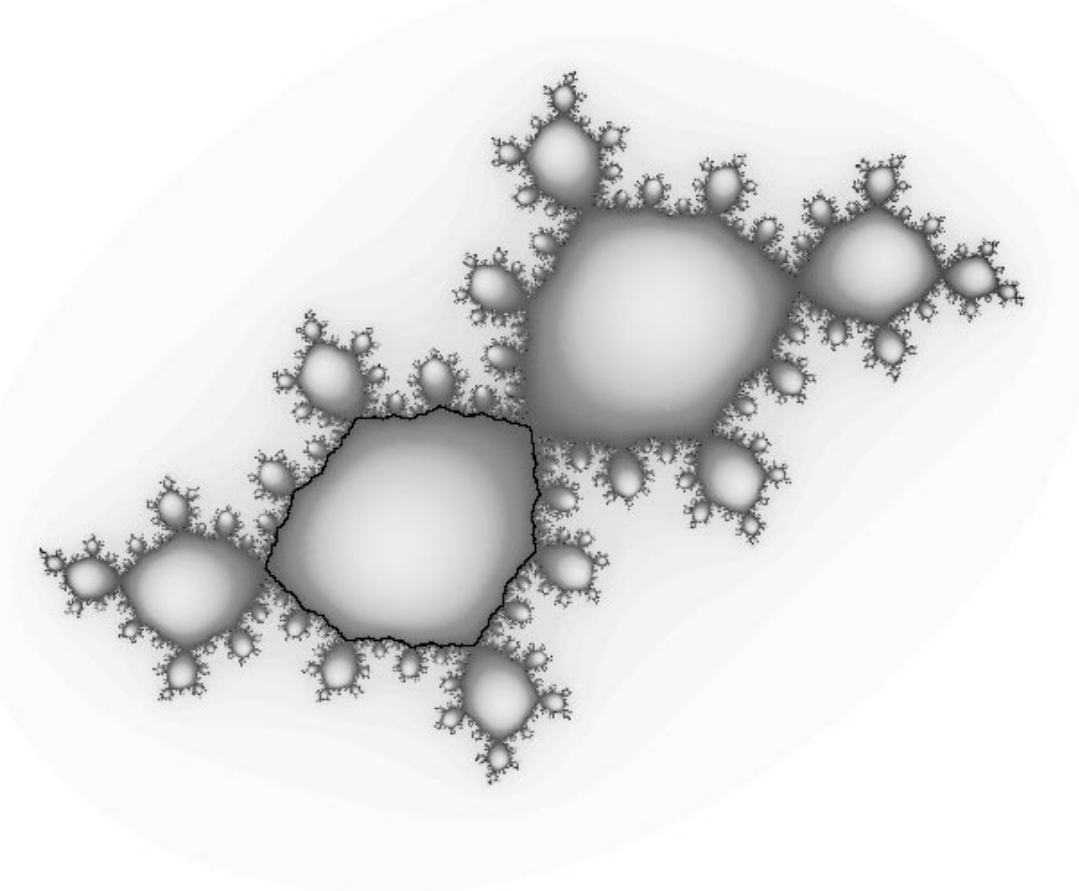

Figure 1. Filled Julia set $K\left(f_{\theta}\right)$ for $\theta=(\sqrt{5}-1) / 2$.

The maximal such linearization domain is a simply-connected neighborhood of $\alpha$ called the Siegel disk of $f_{\theta}$. The following result has recently been proved by Petersen [Pe]:

Theorem (Petersen). Let $0<\theta<1$ be an irrational of bounded type. Then the Julia set of the quadratic polynomial $f_{\theta}$ is locally-connected and has Lebesgue measure zero.

Figure 1 shows the filled Julia set of the quadratic polynomial $f_{\theta}$ for the golden mean $\theta=(\sqrt{5}-1) / 2$.

In proving his theorem, Petersen does not work directly with the Julia set of $f_{\theta}$, but instead considers a certain Blaschke product, which is related to $f_{\theta}$ via a quasiconformal surgery procedure. A simplified version of his argument, based on complex a priori bounds for renormalization of critical circle maps, was presented by one of the authors in [Ya]. Since the Julia set of $f_{\theta}$ is locally-connected, we may pose mateability questions for these polynomials. Our main result is the following theorem:

Main Theorem. Let $0<\theta, \nu<1$ be two irrationals of bounded type and let $\theta \neq 1-\nu$. Then the polynomials $f_{\theta}$ and $f_{\nu}$ are topologically mateable. Moreover, there exists a quadratic rational map $F$ such that

$$
F=f_{\theta} \sqcup f_{\nu} .
$$

Any two such rational maps are conjugate by a Möbius transformation. 


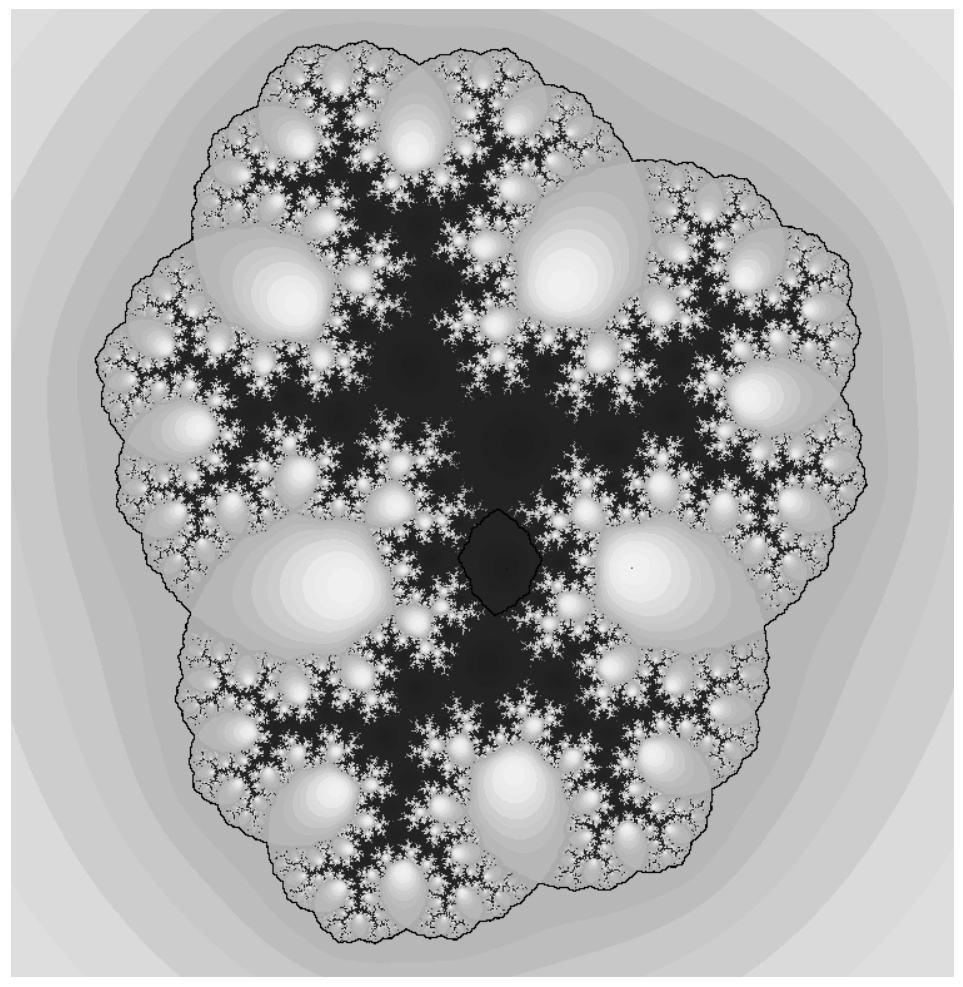

Figure 2. The Julia set of the mating $f_{\theta} \sqcup f_{\theta}$ for $\theta=(\sqrt{5}-1) / 2$.

In other words, one can paste any two filled Julia sets of the type shown in Figure 1 along their boundaries to obtain a 2 -sphere, and the actions of the polynomials on their filled Julia sets match up to give an action on the sphere which is conjugate to a quadratic rational map with two fixed Siegel disks. Figure 2 shows the result of this pasting in the case $\theta=\nu=(\sqrt{5}-1) / 2$. In this picture we normalize the quadratic rational map $f_{\theta} \sqcup f_{\theta}$ to put the centers of the Siegel disks at zero and infinity. The black and gray regions are the images of the copies of the corresponding filled Julia sets in Figure 1. There are, however, some prominent differences between these regions and the original filled Julia sets. First, there are infinitely many "pinch points" in the "ends" of the black and gray regions that are not present in the original filled Julia sets. An explicit combinatorial description of these pinch points will be presented in $\oint 8$ Also, as J. Milnor pointed out to us, an infinite chain of preimages of the Siegel disk in the filled Julia set in Figure 1 which lands at an endpoint in $J\left(f_{\theta}\right)$ maps to a chain in Figure 2 which appears very stretched out near the end. This indicates that the continuous semiconjugacies between the filled Julia sets and their corresponding regions, although conformal in the interior of the sets, have a great amount of distortion near the boundary.

In the case $\theta=1-\nu$ the existence of a mating is ruled out for algebraic reasons. In fact, the polynomials are not even topologically mateable. Under the assumptions of the theorem, the candidate rational map $F$ can be specified algebraically, and the main difficulty lies in establishing that $F$ is indeed a mating. To fix the ideas we may assume that the candidate $F$ has a Siegel disk $\Delta^{0}$ with rotation number 
$\theta$ centered at 0 , and another one $\Delta^{\infty}$ with rotation number $\nu$ centered at $\infty$. There is an unambiguous way to construct the semiconjugacies of Definition IIa in the interiors of the filled Julia sets, by mapping the preimages of the Siegel disk of $f_{\theta}$ to the corresponding preimages of $\Delta^{0}$ and similarly the preimages of the Siegel disk of $f_{\nu}$ to the corresponding preimages of $\Delta^{\infty}$. To guarantee that these semiconjugacies extend continuously to the filled Julia sets we need to demonstrate that the boundaries $\partial \Delta^{0}$ and $\partial \Delta^{\infty}$ are Jordan curves each containing a critical point of $F$ and that the Euclidean diameter of the $n$-th preimages of $\Delta^{0}$ and $\Delta^{\infty}$ goes to zero uniformly in $n$. Proving these properties of the map $F$ directly seems to be quite out of reach. We establish the first property by using a new Blaschke product model for the dynamics of $F$ that was discovered by one of the authors when he was working on dynamics of cubic Siegel polynomials Za2. We then adapt the complex bounds from $\mathrm{Ya}$ to this model to prove the second property. Further properties of the semiconjugacies of Definition IIa are demonstrated by a combinatorial argument using spines and itineraries.

The symmetry of the construction in the case of a self-mating (i.e., when $\theta=\nu$ ) has a nice corollary. In this case the mating $F=f_{\theta} \sqcup f_{\theta}$ given by the Main Theorem commutes with the Möbius involution $\mathcal{I}$ which interchanges the centers of the two Siegel disks and fixes the third fixed point of $F$. Hence one can pass to the quotient Riemann surface $\widehat{\mathbb{C}} / \mathcal{I} \simeq \widehat{\mathbb{C}}$ to obtain a new quadratic rational map $G$. It is not hard to see that $G$ is the mating of $f_{\theta}$ with the Chebyshev quadratic polynomial $f_{\text {cheb }}: z \mapsto z^{2}-2$ whose filled Julia set is the interval $[-2,2]$ :

Theorem. Let $0<\theta<1$ be any irrational of bounded type. Then there exists a quadratic rational map $G$ such that

$$
G=f_{\theta} \sqcup f_{\text {cheb }} .
$$

Moreover, $G$ is unique up to conjugation with a Möbius transformation.

We would like to point out that our main theorem gives an affirmative (partial) answer to the following more general question posed by Milnor in [Mi4]:

Question. Let $f_{c_{1}}$ and $f_{c_{2}}$ be quadratic polynomials on the boundary of the main cardioid of the Mandelbrot set with locally-connected Julia sets and with $c_{1} \neq \overline{c_{2}}$. Does the conformal mating $f_{c_{1}} \sqcup f_{c_{2}}$ exist?

The case of mating two parabolics can now be treated using the parabolic surgery introduced by P. Haïssinsky in $\mathrm{Ha}$. We believe that the techniques developed here, combined with the parabolic surgery, are adequate to handle the matings of parabolics with the bounded type Siegel quadratics. There remain the more challenging cases where the rotation numbers involved fail to be of bounded type.

Acknowledgements. We would like to express our gratitude to John Milnor for posing the problem and encouraging the dynamics group at Stony Brook to look at it. His picture of the "presumed mating of golden ratio Siegel disk with itself" (Figure 2 in this paper) posted in the IMS at Stony Brook was the inspiration for this work. Adam Epstein, who also was enthusiastic about this problem and had learned about our similar ideas, brought the two of us together. We are indebted to him because this joint paper would have never existed without his persistence. The referee made valuable suggestions for certain improvements in our presentation, for which we are thankful. Finally, we gratefully acknowledge the important role that Carsten Petersen's ideas in $\mathrm{Pe}$ play in our work. 


\section{BACKGROUND MATERIAL}

2.1. Notations and terminology. The unit disk in the complex plane will be denoted by $\mathbb{D}$, its boundary is the unit circle $\mathbb{T}$. For a set $X$ in the plane, we use $\bar{X}$ and $\stackrel{\circ}{X}$ for the closure and the interior of $X$, respectively. We use $|J|$ for the length of an interval $J$, and dist and diam for the Euclidean distance and diameter in $\mathbb{C}$. We write $[a, b]$ for the closed interval with endpoints $a$ and $b$ in $\mathbb{R}$ without specifying their order. For a hyperbolic Riemann surface $X$, dist $_{X}$ will denote the distance in the hyperbolic metric in $X$.

Let $K>1$. We say that two real numbers $a$ and $b$ are $K$-commensurable if $K^{-1} \leq|a| /|b| \leq K$. In a given statement or proof, we often drop the explicit dependence on $K$ and simply say that $a$ and $b$ are commensurable, by which we mean that there exists some $K$ such that for all choices of $a, b$ in that context, $a$ and $b$ are $K$-commensurable. Two sets $X$ and $Y$ in $\mathbb{C}$ are $K$-commensurable if their diameters are. A configuration of points $x_{1}, \ldots, x_{n}$ is called $K$-bounded if any two intervals $\left[x_{i}, x_{j}\right]$ and $\left[x_{k}, x_{l}\right]$ are $K$-commensurable. For a pair of intervals $I \subset J$ we say that $I$ is well inside of $J$ if there exists a universal constant $K>0$, such that for each component $L$ of $J \backslash I$ we have $|L| \geq K|I|$.

For two points $a, b$ on the circle which are not diagonally opposite, $[a, b]$ will denote, unless otherwise specified, the shorter of the two closed arcs connecting them. When working with a homeomorphism $f$ of the unit circle, which extends beyond the circle, we will reserve the notation $f^{-i}(z)$ for the $i$-th preimage of $z \in \mathbb{T}$ contained in the circle $\mathbb{T}$.

2.2. Quadratic rational maps. The reader may find a detailed discussion of the dynamics of quadratic rational maps in Milnor's paper [Mi2]. Below we give a brief summary of some relevant facts. A quadratic rational map of the Riemann sphere $\widehat{\mathbb{C}}$ may be expressed as a ratio

$$
F(z)=\frac{a_{0} z^{2}+a_{1} z+a_{2}}{b_{0} z^{2}+b_{1} z+b_{2}}
$$

with one of the coefficients $a_{0}, b_{0}$ different from 0 . The six-tuple $\left(a_{0}: a_{1}: a_{2}: b_{0}\right.$ : $\left.b_{1}: b_{2}\right)$ may be viewed as a point in the complex projective space $\mathbb{C P}^{5}$. The space of all quadratic rational maps $\mathbf{R a t}_{2}$ is identified in this way with a Zariski open subset of $\mathbb{C P}^{5}$ (see [Mi2] for a description of the topology of this set). From the point of view of complex dynamics the quadratic rational maps which are conjugate by a conformal isomorphism of the Riemann sphere are identified. That is, we consider the quotient space of $\mathbf{R a t}_{2}$ by the action of the group Möb $\simeq P S L_{2}(\mathbb{C})$ of Möbius transformations. This moduli space of quadratic rational maps will be denoted $\mathcal{M}_{2}$. The action of Möb on $\mathbf{R a t}_{2}$ is locally free, and the quotient space has the structure of a 2-dimensional complex orbifold branched over a set $\mathcal{S} \subset \mathcal{M}_{2}$. This symmetry locus $\mathcal{S}$ consists of maps possessing a non-trivial automorphism group.

A more useful parametrization of the moduli space $\mathcal{M}_{2}$ comes from the following considerations. Every map $F \in \mathbf{R a t}_{2}$ has three not necessarily distinct fixed points. Let $\mu_{1}, \mu_{2}, \mu_{3}$ denote the multipliers of the fixed points. (By definition, the multiplier of $F$ at a fixed point $p$ is simply the derivative $F^{\prime}(p)$ with appropriate modification if $p=\infty$.) Let

$$
\sigma_{1}=\mu_{1}+\mu_{2}+\mu_{3}, \sigma_{2}=\mu_{1} \mu_{2}+\mu_{1} \mu_{3}+\mu_{2} \mu_{3}, \sigma_{3}=\mu_{1} \mu_{2} \mu_{3}
$$

be the elementary symmetric functions of these multipliers. 
Proposition (Mi2], Lemma 3.1). The numbers $\sigma_{1}, \sigma_{2}, \sigma_{3}$ determine $F$ up to a Möbius conjugacy, and are subject only to the restriction that

$$
\sigma_{3}=\sigma_{1}-2
$$

Hence the moduli space $\mathcal{M}_{2}$ is canonically isomorphic to $\mathbb{C}^{2}$, with coordinates $\sigma_{1}$ and $\sigma_{2}$.

Note that for any choice of $\mu_{1}, \mu_{2}$ with $\mu_{1} \mu_{2} \neq 1$ there exists a quadratic rational map $F$, unique up to a Möbius conjugacy, which has distinct fixed points with these multipliers. The third multiplier can be computed as $\mu_{3}=\left(2-\mu_{1}-\mu_{2}\right) /\left(1-\mu_{1} \mu_{2}\right)$.

As a special case, let $F$ be a quadratic rational map which has two Siegel disks centered at two fixed points of multipliers $e^{2 \pi i \theta}$ and $e^{2 \pi i \nu}$, where $0<\theta, \nu<1$. Note that we necessarily have $\theta \neq 1-\nu$. By conjugating $F$ with a Möbius transformation which sends the two centers to 0 and $\infty$ and the third fixed point to 1 , we obtain a quadratic rational map which fixes $0,1, \infty$ and has multipliers $e^{2 \pi i \theta}$ at 0 and $e^{2 \pi i \nu}$ at $\infty$. It is easy to see that these conditions determine the map uniquely. In fact, we obtain the normal form

$$
F_{\theta, \nu}: z \mapsto z \frac{\left(1-e^{2 \pi i \theta}\right) z+e^{2 \pi i \theta}\left(1-e^{2 \pi i \nu}\right)}{\left(1-e^{2 \pi i \theta}\right) e^{2 \pi i \nu} z+\left(1-e^{2 \pi i \nu}\right)} .
$$

2.3. Critical circle maps. Throughout this paper, we shall identify the unit circle $\mathbb{T}=\{z \in \mathbb{C}:|z|=1\}$ with the affine manifold $\mathbb{R} / \mathbb{Z}$ using the canonical projection from the real line given by $x \mapsto e^{2 \pi i x}$. By definition, a critical circle map is an orientation-preserving homeomorphism of the circle $\mathbb{T}$ of class $C^{3}$ with a single critical point $c$. We further assume that the critical point is of cubic type. This means that for a lift $\hat{f}: \mathbb{R} \rightarrow \mathbb{R}$ of $f$ with critical points at integer translates of $\hat{c}$,

$$
\hat{f}(x)-\hat{f}(\hat{c})=(x-\hat{c})^{3}(\text { const }+O(x-\hat{c})) .
$$

The standard examples of analytic critical circle maps are provided by the projections to $\mathbb{T}$ of homeomorphisms in the Arnold family:

$$
A^{t}: x \mapsto x+t-\frac{1}{2 \pi} \sin 2 \pi x .
$$

Another group of examples, more relevant for our considerations, is given by the family of degree 3 Blaschke products

$$
Q^{t}: z \mapsto e^{2 \pi i t} z^{2}\left(\frac{z-3}{1-3 z}\right) .
$$

The restriction of $Q^{t}$ to the unit circle $\mathbb{T}$ is a real-analytic homeomorphism. Every $Q^{t}$ has a critical point of cubic type at $1 \in \mathbb{T}$ and no other critical points in $\mathbb{T}$, thus $\left.Q^{t}\right|_{\mathbb{T}}$ is a critical circle map.

The quantity

$$
\rho(f)=\lim _{n \rightarrow \infty} \frac{\hat{f}^{\circ n}(x)}{n}(\bmod 1)
$$

is independent both of the choice of $x \in \mathbb{R}$ and the lift $\hat{f}$ of a critical circle map $f$, and is referred to as the rotation number of $f$. The rotation number is rational of the form $\rho(f)=p / q$ if and only if $f$ has an orbit of period $q$. To further illustrate the connection between the number-theoretic properties of $\rho(f)$ and the dynamics of $f$, let us introduce the notion of a closest return of the critical point $c$. The iterate $f^{\circ n}(c)$ is a closest return, or equivalently, $n$ is a closest return moment, if 
the interior of the arc $\left[f^{\circ n}(c), c\right]$ contains no iterates $f^{\circ j}(c)$ with $j<n$. Consider the representation of $\rho(f)$ as a (possibly finite) continued fraction

$$
\rho(f)=\frac{1}{a_{1}+\frac{1}{a_{2}+\frac{1}{a_{3}+\cdots}}},
$$

with the $a_{i}$ positive integers. For convenience we will write $\rho(f)=\left[a_{1}, a_{2}, a_{3}, \ldots\right]$. The $n$-th convergent of the continued fraction of $\rho(f)$ is the rational number

$$
\frac{p_{n}}{q_{n}}=\left[a_{1}, a_{2}, \ldots, a_{n}\right]
$$

written in reduced form. We set $p_{0}=0, q_{0}=1$. One easily verifies the recursive relations

$$
\begin{aligned}
& p_{n}=a_{n} p_{n-1}+p_{n-2}, \\
& q_{n}=a_{n} q_{n-1}+q_{n-2},
\end{aligned}
$$

for $n \geq 2$. In this notation, the iterates $\left\{f^{\circ q_{n}}(c)\right\}$ are the consecutive closest returns of the critical point $c$ (see for example [MvS]).

The rotation number $\rho(f)$ is said to be of bounded type if $\sup a_{i}<\infty$. We will make use of two linearization theorems for critical circle maps. Let us denote by $\varrho_{\theta}$ the rigid rotation $x \mapsto x+\theta(\bmod \mathbb{Z})$. Yoccoz Yo1 has shown:

Theorem. Let $f$ be a critical circle map with irrational rotation number $\theta$. Then there exists a homeomorphic change of coordinates $h: \mathbb{T} \rightarrow \mathbb{T}$ such that

$$
h \circ f \circ h^{-1}=\varrho_{\theta} .
$$

In general the homeomorphism $h$ may not be regular at all, even if the map $f$ is real-analytic. However, some regularity for $h$ can be gained at the expense of extra assumptions on the rotation number $\rho(f)$. The following theorem of Herman He provides us with a sharp result which will be useful later in performing a quasiconformal surgery. Recall that a homeomorphism $h: \mathbb{R} \rightarrow \mathbb{R}$ is called $K$ quasisymmetric if

$$
0<K^{-1} \leq \frac{|h(x+t)-h(x)|}{|h(x)-h(x-t)|} \leq K<+\infty
$$

for all $x$ and all $t>0$. A homeomorphism $h: \mathbb{T} \rightarrow \mathbb{T}$ is $K$-quasisymmetric if its lift to $\mathbb{R}$ is such a homeomorphism. We simply call $h$ quasisymmetric if it is $K$-quasisymmetric for some $K$.

Theorem. A critical circle map $f$ is conjugate to a rigid rotation by a quasisymmetric homeomorphism $h$ if and only if the rotation number $\rho(f)$ is irrational of bounded type.

The above result is based on a set of estimates on the small-scale geometry of critical circle maps. The estimates of this type first appeared in the work of Swiątek [Sw], and were later generalized by Herman. These Światek-Herman real a priori bounds became a key element of renormalization and rigidity results for critical circle maps, and will play an important role in this paper. The paper dFdM contains an excellent exposition of the bounds, which we follow in our presentation. Here are some preliminary definitions. For a critical circle map $f$ with an irrational 
rotation number, we denote by $I_{n}$ the $n$-th closest return interval $\left[c, f^{\circ q_{n}}(c)\right]$. One has no difficulty verifying that for every $n>1$ the closed intervals

$$
I_{n-1}, f\left(I_{n-1}\right), \ldots, f^{\circ q_{n}-1}\left(I_{n-1}\right), I_{n}, f\left(I_{n}\right), \ldots, f^{\circ q_{n-1}-1}\left(I_{n}\right)
$$

cover the entire circle, and have disjoint interiors. By excluding from each of the intervals its right endpoint, according to the standard choice of orientation of $\mathbb{T}$, we obtain a partition of $\mathbb{T}$ which is called the dynamical partition of level $n$ associated to $f$.

Świątek-Herman real a priori bounds. There exists $K>1$ such that for every critical circle map $f$ with an irrational rotation number the following holds: There exists $N=N(f)>0$ such that for every $n>N$ the adjacent elements of the dynamical partition of level $n$ are $K$-commensurable. In particular,

$$
K^{-1}\left|I_{n}\right| \leq\left|I_{n+1}\right| \leq K\left|I_{n}\right| .
$$

Moreover, let $\alpha_{n}: \mathbb{R} \rightarrow \mathbb{R}$ denote the affine map which maps $I_{n-1}$ to $[0,1]$ sending $c$ to 0 , and set $q(z)=z^{3}$. Then, there exists a $C^{2}$-compact family $\mathcal{F}$ of $C^{3}$ diffeomorphisms of the interval $[0,1]$ into $\mathbb{R}$ such that for $n>N$,

$$
\left.\alpha_{n} \circ f^{\circ q_{n}} \circ \alpha_{n}^{-1}\right|_{[0,1]}=H_{n} \circ q \circ h_{n},
$$

where $H_{n} \in \mathcal{F}$ and $h_{n}$ is a $C^{3}$ diffeomorphism of $[0,1]$ with $h_{n} \rightarrow$ id in $C^{2}$-topology.

As a consequence, for every $M>0$ there exists a universal constant $K_{M}>1$ such that the following holds: For all sufficiently large $n$, the $\operatorname{arcs}\left[f^{\circ q_{n-1}+(j-1) q_{n}}(c)\right.$, $\left.f^{\circ q_{n-1}+j q_{n}}(c)\right],\left[f^{-(j-1) q_{n}}(c), f^{-j q_{n}}(c)\right]$ and $\left[c, f^{\circ q_{n-1}}(c)\right]$ are $K_{M}$-commensurable, for $1 \leq j \leq a_{n+1}-1$ with $\min \left(j, a_{n+1}-j\right)<M$.

We conclude this section with a basic fact about the combinatorics of closest returns. Let $\left[a_{1}, a_{2}, \ldots\right]$ be the continued fraction expansion of the irrational rotation number $\rho(f)$ with convergents $p_{n} / q_{n}$. Then (see [dMvS]) the consecutive closest returns $f^{\circ q_{n}}(c)$ and $f^{\circ q_{n+1}}(c)$ occur on different sides of the critical point $c$, that is, $c \in\left(f^{\circ q_{n}}(c), f^{\circ q_{n+1}}(c)\right)$. Below is a list of some of the points in the forward orbit of $c$ in the order they are encountered when going from $f^{\circ q_{n-1}}(c)$ to $f^{q_{n}}(c)$ :

$$
\begin{gathered}
f^{\circ q_{n-1}}(c), f^{\circ q_{n-1}+q_{n}}(c), f^{\circ q_{n-1}+2 q_{n}}(c), \ldots, f^{\circ q_{n-1}+a_{n+1} q_{n}}(c) \\
=f^{\circ q_{n+1}}(c), c, f^{-q_{n+1}}(c), f^{\circ q_{n}}(c) .
\end{gathered}
$$

\section{The Blaschke model for Petersen's theorem}

As a motivation for further discussion, we present with slight modifications the construction of a Blaschke product model for a Siegel quadratic polynomial used by Petersen in $[\mathrm{Pe}$. Much of the tools developed in this section will carry over to the Blaschke product model for mating introduced in $\$$ It is somewhat easier, however, to discuss them in this context. Let us define

$$
Q^{t}: z \mapsto e^{2 \pi i t} z^{2}\left(\frac{z-3}{1-3 z}\right)
$$

As we have seen in the previous section, the restriction $\left.Q^{t}\right|_{\mathbb{T}}$ is a critical circle map with critical value $t \in \mathbb{T}$. The standard monotonicity considerations imply that for each irrational number $0<\theta<1$ there exists a unique value $t(\theta)$ for which the rotation number $\rho\left(\left.Q^{t(\theta)}\right|_{\mathbb{T}}\right)$ is $\theta$. Let us set $Q_{\theta}=Q^{t(\theta)}$. 
3.1. Elementary properties. For the moment, let us work with a fixed irrational $\theta$ and abbreviate $Q=Q_{\theta}$. As seen from (3.1), $Q$ has superattracting fixed points at 0 and $\infty$ and a double critical point at $z=1$. The immediate basin of attraction of infinity, which we denote by $A(\infty)$, is a simply-connected region on which $Q$ acts as a degree 2 branched covering. $Q$ commutes with the reflection $\mathcal{T}: z \mapsto 1 / \bar{z}$ through $\mathbb{T}$, so we have a similar description for $A(0)=\mathcal{T}(A(\infty))$, the immediate basin of attraction of the origin.

Just as in the polynomial case, there exists a unique conformal isomorphism $\varphi: A(\infty) \stackrel{\simeq}{\longrightarrow} \widehat{\mathbb{C}} \backslash \overline{\mathbb{D}}$ with $\varphi(\infty)=\infty$ and $\varphi^{\prime}(\infty)=1$, which conjugates $\varphi$ on $A(\infty)$ to the squaring map $z \mapsto z^{2}$ on $\widehat{\mathbb{C}} \backslash \overline{\mathbb{D}}$. We may use it to define the external rays $R^{e}(t)=\varphi^{-1}\left\{r e^{2 \pi i t}: r>1\right\}$ for $t \in \mathbb{T}$, and the equipotentials $E_{r}=\varphi^{-1}\left\{r e^{2 \pi i t}: t \in\right.$ $\mathbb{T}\}$ for $r>1$. The ray $R^{e}(t)$ lands at $p$ if $\lim _{r \rightarrow 1} \varphi^{-1}\left(r e^{2 \pi i t}\right)=p$.

Proposition 3.1. $A(\infty)=\widehat{\mathbb{C}} \backslash \overline{\bigcup_{n \geq 0} Q^{-n}(\mathbb{D})}$.

Proof. Let us put $U=\widehat{\mathbb{C}} \backslash \overline{\bigcup_{n \geq 0} Q^{-n}(\mathbb{D})}$. Clearly $A(\infty) \subset U$ and $Q(U) \subset U$. Since $\overline{\bigcup_{n \geq 0} Q^{-n}(\mathbb{T})}=J(Q), U$ is a subset of the Fatou set of $Q$. Assume by way of contradiction that $A(\infty) \neq U$. Then there must be a connected component of $U$ other than $A(\infty)$ which eventually maps to a periodic Fatou component $V$ by Sullivan's No Wandering Theorem. We have $V \neq A(\infty)$, since otherwise $Q$ would have to have a pole $\neq \infty$ in $U$. According to Fatou-Sullivan, $V$ is either the attracting basin of an attracting or parabolic periodic point, or a Siegel disk or a Herman ring. In the first two cases, there must be a critical point in $V$ which converges to the periodic orbit. But $V \subset \mathbb{C} \backslash \overline{\mathbb{D}}$ and there is no critical point of $Q$ in $\mathbb{C} \backslash \overline{\mathbb{D}}$. In the last two cases, some critical point in $J(Q)$ must accumulate on the boundary of the Siegel disk or Herman ring. The only critical point in $J(Q)$ is $z=1$ whose forward orbit is dense on the unit circle $\mathbb{T}$. It follows that $\mathbb{T}$ must be the boundary of the Siegel disk or a component of the boundary of the Herman ring. Evidently this is impossible since by the Reflection Principle such a boundary can never be real-analytic.

By the theorem of Yoccoz (see subsection 2.3), there exists a unique homeomorphism $h: \mathbb{T} \rightarrow \mathbb{T}$ with $h(1)=1$ such that $\left.h \circ Q\right|_{\mathbb{T}}=\varrho_{\theta} \circ h$, where $\varrho_{\theta}: z \mapsto e^{2 \pi i \theta} z$ is the rigid rotation by angle $\theta$. Let $H: \mathbb{D} \rightarrow \mathbb{D}$ be a homeomorphic extension of $h$ to the unit disk. To have a canonical homeomorphism at hand, we assume that $H$ is given by the Douady-Earle extension of circle homeomorphisms [DE]. Define a modified Blaschke product

$$
\tilde{Q}(z)=\tilde{Q}_{\theta}(z)= \begin{cases}Q(z), & |z| \geq 1 \\ \left(H^{-1} \circ \varrho_{\theta} \circ H\right)(z), & |z| \leq 1\end{cases}
$$

where the two definitions match along the boundary of $\mathbb{D}$. Evidently, $\tilde{Q}$ is a degree 2 branched covering of the sphere which is holomorphic outside of the unit disk and is topologically conjugate to a rigid rotation on the unit disk. Imitating the polynomial case, we define the "filled Julia set" of $\tilde{Q}$ by

$$
K(\tilde{Q})=\left\{z \in \mathbb{C}: \text { the orbit }\left\{\tilde{Q}^{\circ n}(z)\right\}_{n \geq 0} \text { is bounded }\right\}
$$

and the "Julia set" of $\tilde{Q}$ as the topological boundary of $K(\tilde{Q})$ :

$$
J(\tilde{Q})=\partial K(\tilde{Q})
$$




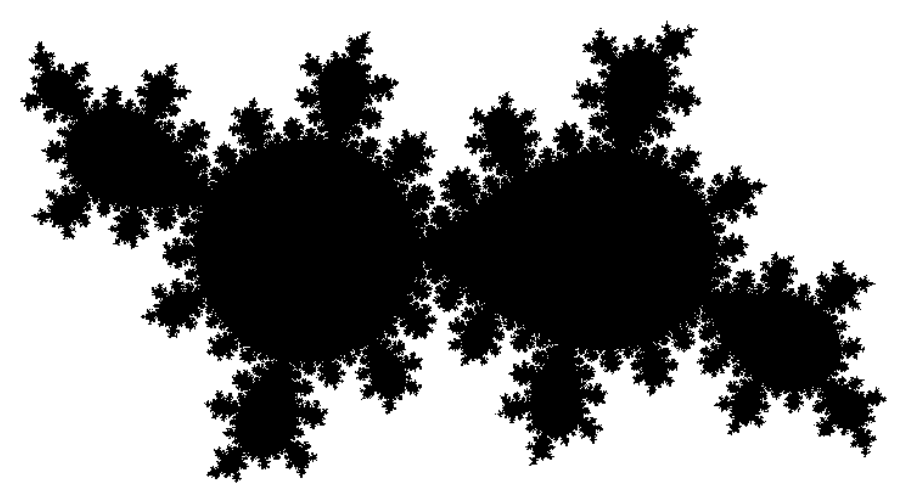

Figure 3. "Filled Julia set" $K\left(\tilde{Q}_{\theta}\right)$ for $\theta=(\sqrt{5}-1) / 2$.

By Proposition 3.1 we have

$$
K(\tilde{Q})=\widehat{\mathbb{C}} \backslash A(\infty), \quad J(\tilde{Q})=\partial A(\infty) .
$$

In particular, $K(\tilde{Q})$ is full. Figure 3 shows the set $K(\tilde{Q})$ for the golden mean $\theta=(\sqrt{5}-1) / 2$. In this case, $t(\theta)=0.613648 \ldots$

3.2. Drops and their addresses. In what follows we collect basic facts about the "drops" associated with $\tilde{Q}$ and their addresses (see [Pe], and compare [Za2] for a more general notion of drops in a similar family of degree 5 Blaschke products). By definition, the unit disk $\mathbb{D}$ is called the 0 -drop of $\tilde{Q}$ and the unique component $U_{1}=\tilde{Q}^{-1}(\mathbb{D}) \backslash \mathbb{D}$ is called the 1 -drop of $\tilde{Q}$. This is the large Jordan domain attached to the unit disk at $x=1$ (see Figure [3). More generally, for $n \geq 2$, any component $U$ of $\tilde{Q}^{-(n-1)}\left(U_{1}\right)$ is a Jordan domain called an $n$-drop, with $n$ the depth of $U$. The map $\tilde{Q}^{\circ n}=Q^{\circ n}: U \rightarrow \mathbb{D}$ is a conformal isomorphism. The unique point $z=z(U) \in U$ with the property $\tilde{Q}^{\circ n}(z)=H^{-1}(0)$ is called the center of $U$. This is the point in $U$ which eventually maps to the fixed point $H^{-1}(0)$ of the topological rotation $\tilde{Q}: \mathbb{D} \rightarrow \mathbb{D}$. The unique point $\tilde{Q}^{-n}(1) \cap \partial U$ is called the root of $U$ and is denoted by $x(U)$. The boundary $\partial U$ is a real-analytic Jordan curve except at the root where it has a definite angle $\pi / 3$. We simply refer to $U$ as a drop when the depth is not important for us.

Let $U$ and $V$ be two drops of depths $m$ and $n$, respectively. Then either $\bar{U} \cap \bar{V}=\emptyset$, or else $\bar{U}$ and $\bar{V}$ intersect at a unique point, in which case we necessarily have $m \neq n$. If we assume for example that $m<n$, then it is easy to check that $\bar{U} \cap \bar{V}=x(V)$. When this is the case, we call $U$ the parent of $V$, or $V$ a child of $U$. It is not hard to check that every $n$-drop with $n \geq 1$ has a unique parent which is an $m$-drop with $0 \leq m<n$. In particular the root of this $n$-drop belongs to the boundary of its parent.

By definition, $\mathbb{D}$ is said to be of generation 0 . Any child of $\mathbb{D}$ is of generation 1 . In general, a drop is of generation $k$ if and only if its parent is of generation $k-1$. 
Lemma 3.2 (Roots determine children). Given a point $p \in \bigcup_{n \geq 0} \tilde{Q}^{-n}(1)$, there exists a unique drop $U$ with $x(U)=p$. In particular, two distinct children of a parent have distinct roots.

Proof. It suffices to show that $U_{1}$ is the only child of $\mathbb{D}$ whose root is $z=1$. Suppose that $U \neq U_{1}$ is an $n$-drop with $x(U)=1$. Then $\tilde{Q}^{\circ n-1}(U)=U_{1}$ implies $\tilde{Q}^{\circ n-1}(x(U))=x\left(U_{1}\right)$, or $\tilde{Q}^{\circ n-1}(1)=1$. Since $n>1$ by the assumption, this contradicts the fact that the rotation number of $\left.\tilde{Q}\right|_{\mathbb{T}}=\left.Q\right|_{\mathbb{T}}$ is irrational.

We give a symbolic description of various drops by assigning an address to every drop. This is a slightly modified version of Petersen's approach, based on a suggestion of J. Milnor. Set $U_{0}=\mathbb{D}$. For $n \geq 1$, let $x_{n}=\tilde{Q}^{-n+1}(1) \cap \mathbb{T}$ and let $U_{n}$ be the $n$-drop with root $x_{n}$, which is well defined by Lemma 3.2 Now let $\iota=\iota_{1} \iota_{2} \cdots \iota_{k}$ be any multi-index of length $k$, where each $\iota_{j}$ is a positive integer. We define the $\left(\iota_{1}+\iota_{2}+\cdots+\iota_{k}\right)$-drop $U_{\iota_{1} \iota_{2} \cdots \iota_{k}}$ of generation $k$ with root

$$
x\left(U_{\iota_{1} \iota_{2} \cdots \iota_{k}}\right)=x_{\iota_{1} \iota_{2} \cdots \iota_{k}}
$$

as follows. We have already defined these for $k=1$. For the induction step, suppose that we have defined $x_{\iota_{1} \iota_{2} \cdots \iota_{k-1}}$ for all multi-indices $\iota_{1} \iota_{2} \cdots \iota_{k-1}$ of length $k-1$. Then, we define

$$
x_{\iota_{1} \iota_{2} \cdots \iota_{k}}= \begin{cases}\tilde{Q}^{-1}\left(x_{\iota_{2} \cdots \iota_{k}}\right) \cap \partial U_{\iota_{1} \iota_{2} \cdots \iota_{k-1}} & \text { if } \iota_{1}=1, \\ \tilde{Q}^{-1}\left(x_{\left(\iota_{1}-1\right) \iota_{2} \cdots \iota_{k}}\right) \cap \partial U_{\iota_{1} \iota_{2} \cdots \iota_{k}-1} & \text { if } \iota_{1}>1 .\end{cases}
$$

Note that the first line of (3.4) defines all the roots of the form $x_{1 \iota_{2} \cdots \iota_{k}}$ and the second line defines all the roots $x_{\iota_{1} \iota_{2} \cdots \iota_{k}}$ by induction on $\iota_{1}$. The corresponding drops $U_{\iota_{1} \iota_{2} \cdots \iota_{k}}$ will then be determined by (3.3) and Lemma 3.2 (see Figure 4).

By the way these drops are given addresses, we have

$$
\tilde{Q}\left(U_{\iota_{1} \iota_{2} \cdots \iota_{k}}\right)= \begin{cases}U_{\iota_{2} \cdots \iota_{k}} & \text { if } \iota_{1}=1 \\ U_{\left(\iota_{1}-1\right) \iota_{2} \cdots \iota_{k}} & \text { if } \iota_{1}>1\end{cases}
$$

3.3. Limbs and wakes. Let us fix a drop $U_{\iota_{1} \cdots \iota_{k}}$. By definition, the $\operatorname{limb} L_{\iota_{1} \cdots \iota_{k}}$ is the closure of the union of this drop and all its descendants (i.e., children and grandchildren, etc.):

$$
L_{\iota_{1} \cdots \iota_{k}}=\overline{\bigcup U_{\iota_{1} \cdots \iota_{k} \cdots}}
$$

Note that $L_{0}=K(\tilde{Q})$. If $\iota_{1} \cdots \iota_{k} \neq 0$, we call $x_{\iota_{1} \cdots \iota_{k}}$ the root of $L_{\iota_{1} \cdots \iota_{k}}$.

It is not immediately clear from this definition that limbs provide a useful partition of the filled Julia set $K(\tilde{Q})$. Indeed, it may happen a priori that the boundary of a limb $\neq L_{0}$ is the whole $J(\tilde{Q})$. This is ruled out by the following key lemma of Petersen $[\mathrm{Pe}]$

Lemma 3.3 (Only two rays). Suppose that $0<\theta<1$ is an irrational number. Then the critical point $z=1$ of $Q_{\theta}$ is the landing point of two and only two external rays $R^{e}(t)$ and $R^{e}(s)$ in $A(\infty)$.

Let $W_{1}$ denote the connected component of $\mathbb{C} \backslash\left(R^{e}(t) \cup R^{e}(s) \cup\{1\}\right)$ containing the drop $U_{1}$. We call $W_{1}$ the wake with root $x_{1}$. Given an arbitrary multi-index $\iota_{1} \cdots \iota_{k}$, we define the wake $W_{\iota_{1} \cdots \iota_{k}}$ as the appropriate pull-back of $W_{1}$. More precisely, consider the two external rays landing at $x_{\iota_{1} \cdots \iota_{k}}$ which map to $R^{e}(t)$ and $R^{e}(s)$ under $\tilde{Q}^{\circ n}$, where $n=\iota_{1}+\cdots+\iota_{k}$. These rays separate the plane into two 


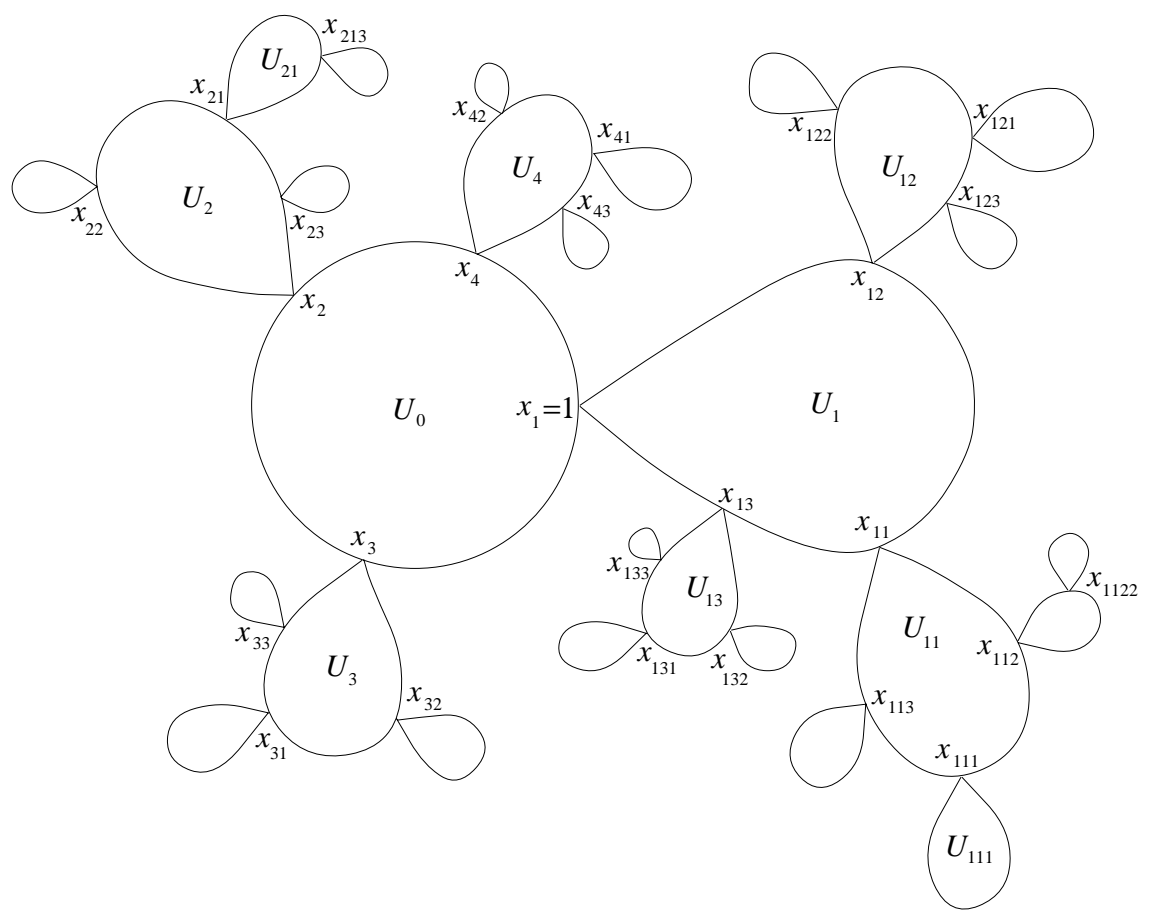

Figure 4. Examples of some drops and their addresses.

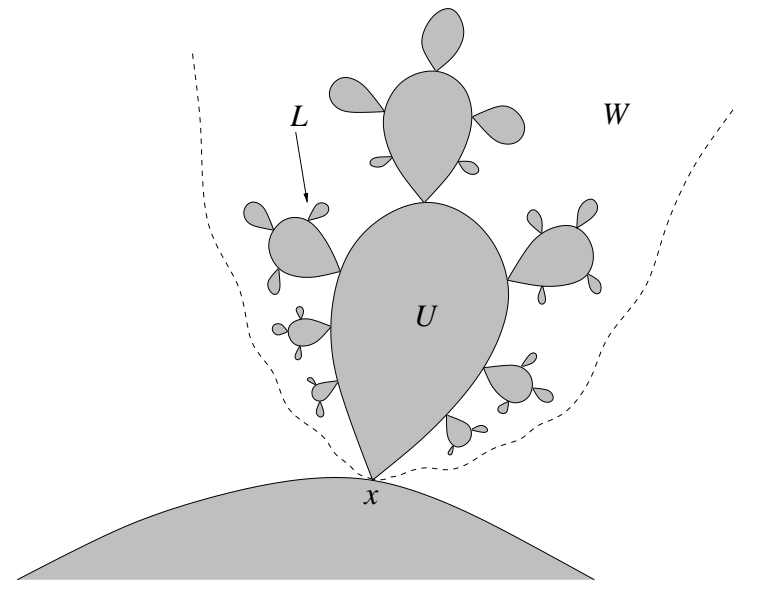

Figure 5. A drop $U$ with $\operatorname{root} x$, and the associated limb $L$ and wake $W$.

simply-connected regions. The wake $W_{\iota_{1} \cdots \iota_{k}}$ will then be the region containing the drop $U_{\iota_{1} \cdots \iota_{k}}$. It is immediately clear that

$$
L_{\iota_{1} \cdots \iota_{k}}=\bar{W}_{\iota_{1} \cdots \iota_{k}} \cap K(\tilde{Q})
$$

(see Figure 5). The integers $n$ and $k$ are respectively called the depth and generation of $W_{\iota_{1} \cdots \iota_{k}}$ as well as $L_{\iota_{1} \cdots \iota_{k}}$. 
The next proposition follows directly from the above definitions:

Proposition 3.4 (Properties of limbs and wakes). Consider $\tilde{Q}_{\theta}$ for an irrational number $0<\theta<1$. Then

(i) If a drop $U$ is contained in a limb $L$, then any child of $U$ is also contained in $L$.

(ii) Any two limbs and any two wakes are either disjoint or nested.

(iii) For any limb $L_{\iota_{1} \cdots \iota_{k}}$, we have

$$
\tilde{Q}_{\theta}\left(L_{\iota_{1} \cdots \iota_{k}}\right)= \begin{cases}L_{\iota_{2} \cdots \iota_{k}} & \text { if } \iota_{1}=1 \\ L_{\left(\iota_{1}-1\right) \iota_{2} \cdots \iota_{k}} & \text { if } \iota_{1}>1 .\end{cases}
$$

In particular, every limb eventually maps to $L_{1}$ and then to the entire filled Julia set $L_{0}=K\left(\tilde{Q}_{\theta}\right)$. The same relation holds for wakes.

The following theorem is a central result of $[\mathrm{Pe}]$.

Theorem 3.5 (Local-connectivity). Suppose that $0<\theta<1$ is an irrational number. Then as the depth of a limb $L$ of $\tilde{Q}_{\theta}$ goes to infinity, the Euclidean diameter $\operatorname{diam}(L)$ goes to 0 . This implies that the Julia set $J\left(Q_{\theta}\right)$, hence $J\left(\tilde{Q}_{\theta}\right)$, is locallyconnected.

One important implication of this result is the non-existence of the so-called "ghost limbs":

Corollary 3.6 (No ghost limbs). Suppose that $0<\theta<1$ is an irrational number. Then the filled Julia set $K\left(\tilde{Q}_{\theta}\right)$ is the union of $\overline{\mathbb{D}}$ and all the limbs of generation 1 :

$$
K\left(\tilde{Q}_{\theta}\right)=\overline{\mathbb{D}} \cup \bigcup_{n \geq 1} L_{n}
$$

This follows from the fact that distinct $L_{n}$ 's are separated by their wakes and $\operatorname{diam}\left(L_{n}\right) \rightarrow 0$ as $n \rightarrow \infty$.

\subsection{Drop-chains.}

Definition 3.7. Consider a sequence of drops $\left\{U_{0}=\mathbb{D}, U_{\iota_{1}}, U_{\iota_{1} \iota_{2}}, U_{\iota_{1} \iota_{2} \iota_{3}}, \ldots\right\}$ where each $U_{\iota_{1} \cdots \iota_{k}}$ is the parent of $U_{\iota_{1} \cdots \iota_{k+1}}$. The closure of the union

$$
\mathcal{C}=\overline{\bigcup_{k} U_{\iota_{1} \cdots \iota_{k}}}
$$

is called a drop-chain.

Consider the corresponding limbs

$$
K(\tilde{Q})=L_{0} \supset L_{\iota_{1}} \supset L_{\iota_{1} \iota_{2}} \supset L_{\iota_{1} \iota_{2} \iota_{3}} \supset \cdots
$$

which are nested by Proposition [3.4. Since $\operatorname{diam}\left(L_{\iota_{1} \cdots \iota_{k}}\right) \rightarrow 0$ as $k \rightarrow \infty$ by Theorem 3.5 the intersection of these limbs must be a unique point which we denote by $p(\mathcal{C})$ :

$$
p(\mathcal{C})=\bigcap_{k} L_{\iota_{1} \cdots \iota_{k}}
$$

Intuitively, $p(\mathcal{C})$ is the unique point in the Julia set of $\tilde{Q}$ to which the tail of $\mathcal{C}$ must converge. It follows that

$$
\mathcal{C}=\bigcup_{k} \bar{U}_{\iota_{1} \cdots \iota_{k}} \cup\{p\}
$$




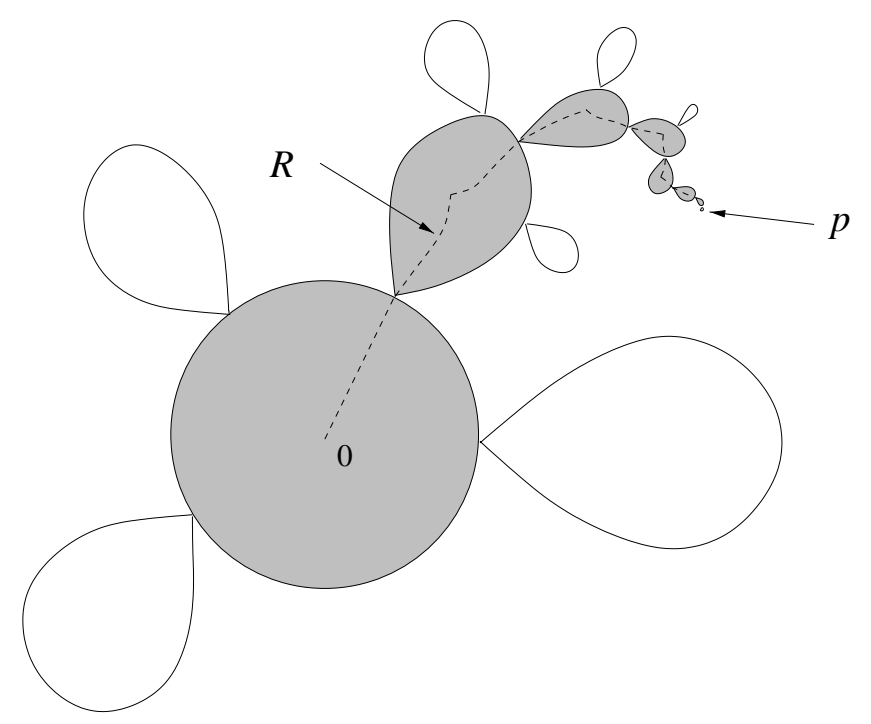

Figure 6. A drop-chain and the drop-ray associated with it.

In particular, $\mathcal{C}$ is compact, connected and locally-connected.

By a ray in a drop $U$ we mean a hyperbolic geodesic which connects some boundary point $p \in \partial U$ to the center $z(U)$. This ray is denoted by $\llbracket p, z(U) \rrbracket=\llbracket z(U), p \rrbracket$. For two distinct points $p, q \in \partial U$, we use the notation $\llbracket p, q \rrbracket$ for the union of the rays $\llbracket p, z(U) \rrbracket \cup \llbracket z(U), q \rrbracket$.

Given any drop-chain $\mathcal{C}$, there exists a unique "most efficient" path $R=R(\mathcal{C})$ in $\mathcal{C}$ which connects 0 to $p(\mathcal{C})$. In fact, if $\mathcal{C}$ is of the form $\overline{\bigcup_{k} U_{\iota_{1} \cdots \iota_{k}}}$, we define

$$
R(\mathcal{C})=\llbracket 0, x_{\iota_{1}} \rrbracket \cup \bigcup_{k \geq 1} \llbracket x_{\iota_{1} \cdots \iota_{k}}, x_{\iota_{1} \cdots \iota_{k+1}} \rrbracket \cup\{p(\mathcal{C})\}
$$

(see Figure 6). It is easy to see that $R(\mathcal{C})$ is a piecewise analytic embedded arc in the plane. We call $R(\mathcal{C})$ the drop-ray associated with $\mathcal{C}$. We often say that $R(\mathcal{C})$, or $\mathcal{C}$, lands at $p(\mathcal{C})$.

Proposition 3.8. Every point in the filled Julia set $K\left(\tilde{Q}_{\theta}\right)$ either belongs to the closure of a drop or is the landing point of a unique drop-chain.

Proof. Let $p \in K\left(\tilde{Q}_{\theta}\right)$ and assume that $p$ does not belong to the closure of any drop. Then by Corollary 3.6 $p$ belongs to some limb $L_{\iota_{1}}$, and inductively, it follows that it belongs to the intersection of a decreasing sequence of limbs $L_{\iota_{1}} \supset L_{\iota_{1} \iota_{2}} \supset L_{\iota_{1} \iota_{2} \iota_{3}} \supset$ $\cdots$. Hence $p$ is the landing point of the corresponding drop-chain $\mathcal{C}=\overline{\bigcup_{k} U_{\iota_{1} \cdots \iota_{k}}}$. Uniqueness of this drop-chain follows from Proposition 3.9 below.

It follows from the next proposition that the union of drop-rays associated with all drop-chains has the structure of an infinite topological tree in which all vertices (corresponding to centers of various drops) have infinite degree.

Proposition 3.9. The assignment $\mathcal{C} \mapsto p(\mathcal{C})$ is one-to-one. In other words, different drop-rays land at distinct points. 
Proof. Suppose that $\mathcal{C}_{1}$ and $\mathcal{C}_{2}$ are two distinct drop-chains. Let $U_{\iota_{1} \cdots \iota_{k}} \subset \mathcal{C}_{1}$ be the drop of smallest generation $k$ which is disjoint from $\mathcal{C}_{2}$, and similarly define $U_{\iota_{1}^{\prime} \cdots \iota_{k}^{\prime}} \subset \mathcal{C}_{2}$. The limbs $L_{\iota_{1} \cdots \iota_{k}}$ and $L_{\iota_{1}^{\prime} \cdots \iota_{k}^{\prime}}$ are disjoint by Proposition 3.4. Since $p\left(\mathcal{C}_{1}\right) \in L_{\iota_{1} \cdots \iota_{k}}$ and $p\left(\mathcal{C}_{2}\right) \in L_{\iota_{1}^{\prime} \cdots \iota_{k}^{\prime}}$, we will have $p\left(\mathcal{C}_{1}\right) \neq p\left(\mathcal{C}_{2}\right)$.

3.5. Surgery. The modified Blaschke product $\tilde{Q}=\tilde{Q}_{\theta}$ as defined in (3.2) is a degree 2 branched covering of the sphere. When the rotation number $\theta$ is irrational of bounded type, the action of $\tilde{Q}_{\theta}$ is in fact conjugate to that of a quadratic polynomial. This follows from a quasiconformal surgery due to Douady, Ghys, Herman, and Shishikura [Do3].

Let us fix an irrational number $0<\theta<1$ of bounded type. By Herman's Theorem (see subsection 2.3) the unique homeomorphism $h: \mathbb{T} \rightarrow \mathbb{T}$ with $h(1)=1$ which conjugates $\left.Q\right|_{\mathbb{T}}$ to $\varrho_{\theta}$ is quasisymmetric. In this case, the Douady-Earle extension $H: \mathbb{D} \rightarrow \mathbb{D}$ of $h$ is a quasiconformal homeomorphism whose dilatation only depends on the dilatation of $h[\mathrm{DE}]$. The modified Blaschke product $\tilde{Q}_{\theta}$ of (3.2) is then a quasiregular branched covering of the sphere. We define a $\tilde{Q}_{\theta^{-}}$ invariant conformal structure $\sigma_{\theta}$ on the plane as follows: On $\mathbb{D}$, let $\sigma_{\theta}$ be the pull-back $H^{*} \sigma_{0}$ of the standard conformal structure $\sigma_{0}$. Since $\varrho_{\theta}$ preserves $\sigma_{0}$, $\tilde{Q}_{\theta}$ will preserve $\sigma_{\theta}$ on $\mathbb{D}$. For every $n \geq 1$, pull $\left.\sigma_{\theta}\right|_{\mathbb{D}}$ back by $\tilde{Q}_{\theta}^{\circ n}=Q_{\theta}^{\circ n}$ on the union of all drops of $\tilde{Q}_{\theta}$ of depth $n$. Since $\tilde{Q}_{\theta}$ is holomorphic, this does not increase the dilatation of $\sigma_{\theta}$. Finally, let $\sigma_{\theta}=\sigma_{0}$ on the rest of the plane. By construction, $\sigma_{\theta}$ has bounded dilatation and is invariant under $\tilde{Q}_{\theta}$. Therefore, by the Measurable Riemann Mapping Theorem (see for example [AB]), we can find a unique quasiconformal homeomorphism $\psi_{\theta}: \widehat{\mathbb{C}} \rightarrow \widehat{\mathbb{C}}$, normalized by $\psi_{\theta}(\infty)=\infty$, $\psi_{\theta}\left(H^{-1}(0)\right)=e^{2 \pi i \theta} / 2$ and $\psi_{\theta}(1)=0$, such that $\psi_{\theta}^{*} \sigma_{0}=\sigma_{\theta}$. Set

$$
f_{\theta}=\psi_{\theta} \circ \tilde{Q}_{\theta} \circ \psi_{\theta}^{-1} .
$$

Then $f_{\theta}$ is a quasiregular self-map of the sphere which preserves $\sigma_{0}$, hence it is holomorphic. Also $f_{\theta}: \mathbb{C} \rightarrow \mathbb{C}$ is a proper map of degree 2 since $\tilde{Q}_{\theta}$ has the same properties. Therefore $f_{\theta}$ is a quadratic polynomial.

Since the action of $f_{\theta}$ on $\psi_{\theta}(\mathbb{D})$ is quasiconformally conjugate to a rigid rotation, $\psi_{\theta}(\mathbb{D})$ is contained in a Siegel disk for $f_{\theta}$ with rotation number $\theta$. As $\psi_{\theta}(1)=0$ is a critical point for $f_{\theta}$, it follows that the entire orbit $\left\{f_{\theta}^{\circ n}(0)\right\}_{n \geq 0}$ lies on the boundary of this Siegel disk. But $\left\{f_{\theta}^{\circ n}(0)\right\}_{n \geq 0}$ is dense on $\psi_{\theta}(\mathbb{T})$, so $\psi_{\theta}(\mathbb{T})$ is exactly the boundary of this Siegel disk, which is a quasicircle passing through the critical point 0 of $f_{\theta}$. Up to affine conjugacy there is only one quadratic polynomial with a fixed Siegel disk of the given rotation number $\theta$. By the way we normalized $\psi_{\theta}$, we must have $f_{\theta}: z \mapsto z^{2}+c_{\theta}$ as in (1.1).

We summarize the above as follows:

Theorem 3.10 (Douady, Ghys, Herman, Shishikura). Let $f$ be a quadratic polynomial which has a fixed Siegel disk $\Delta$ of rotation number $\theta$. If $\theta$ is of bounded type, then $f$ is quasiconformally conjugate to $\tilde{Q}_{\theta}$ in (3.2). In particular, $\partial \Delta$ is a quasicircle passing through the critical point of $f$.

In particular, this surgery allows us to define drops, limbs, wakes, drop-chains and drop-rays for the quadratic polynomial $f_{\theta}$. 


\section{A Blaschke model For mating}

The object of this section is to construct, for a pair of numbers $0<\theta, \nu<1$ with $\theta \neq 1-\nu$, a Blaschke product $B_{\theta, \nu}$. When $\theta$ and $\nu$ are irrationals of bounded type, $B_{\theta, \nu}$ plays the role of a model for the quadratic rational map $F_{\theta, \nu}$ of (2.1) in the same way as $Q_{\theta}$ does for the quadratic polynomial $f_{\theta}$. After showing the existence of such $B_{\theta, \nu}$, we will define drops, limbs, drop-chains and drop-rays for the "modified" $\tilde{B}_{\theta, \nu}$ in an analogous way.

4.1. Existence. We would like to prove the following result:

Theorem 4.1 (Existence of Blaschke models for mating). Let $0 \leq \theta<1,0 \leq \nu<$ 1 and $\theta \neq 1-\nu$. Then there exists a degree 3 Blaschke product

$$
B=B_{\theta, \nu}: z \mapsto \frac{e^{-2 \pi i \nu}}{a b} z\left(\frac{z-a}{1-\bar{a} z}\right)\left(\frac{z-b}{1-\bar{b} z}\right)
$$

with the following properties:

(i) $0<|a|<1$ and $|b|=|a|^{-1}>1$, with $a \bar{b} \neq 1$,

(ii) $B$ has a double critical point at $z=1$, and

(iii) the restriction $\left.B\right|_{\mathbb{T}}$ is a critical circle map with rotation number $\theta$.

The proof of this theorem will be given in the rest of this subsection. In (i) the condition $a \bar{b} \neq 1$ is necessary simply because when $a \bar{b}=1, B$ reduces to the linear map $z \mapsto e^{-2 \pi i \nu} z$.

For simplicity, let us set

$$
\begin{aligned}
& \kappa=a b, \text { where }|\kappa|=1 \text { by (i), } \\
& \zeta=a+b .
\end{aligned}
$$

Using (4.1), the condition $B^{\prime}(z)=0$ may be written in the form

$$
A_{1} z^{4}+A_{2} z^{3}+A_{3} z^{2}+\bar{A}_{2} z+\bar{A}_{1}=0,
$$

where

$$
\begin{aligned}
& A_{1}=\bar{a} \bar{b}=\bar{\kappa}, \\
& A_{2}=-2(\bar{a}+\bar{b})=-2 \bar{\zeta} \\
& A_{3}=2+|a+b|^{2}=2+|\zeta|^{2} .
\end{aligned}
$$

A brief computation shows that the condition of $z=1$ being a double critical point of $B$ translates into

$$
\left\{\begin{array}{l}
4 A_{1}+3 A_{2}+2 A_{3}=-\bar{A}_{2} \\
3 A_{1}+2 A_{2}+A_{3}=\bar{A}_{1}
\end{array}\right.
$$

or by (4.3)

$$
\left\{\begin{array}{l}
2 \kappa-3 \zeta+2+|\zeta|^{2}=\bar{\zeta} \\
3 \kappa-4 \zeta+2+|\zeta|^{2}=\bar{\kappa} .
\end{array}\right.
$$

Subtracting the second equation in (4.4) from the first equation, we find that

$$
\zeta-\kappa=\bar{\zeta}-\bar{\kappa} \Longrightarrow \zeta-\kappa \in \mathbb{R} \text {. }
$$

Set $\kappa=x+i y$ and $\zeta=u+i y$ and substitute them into the first equation in (4.4) to obtain

$$
u^{2}-4 u+\left(2 x+y^{2}+2\right)=0
$$




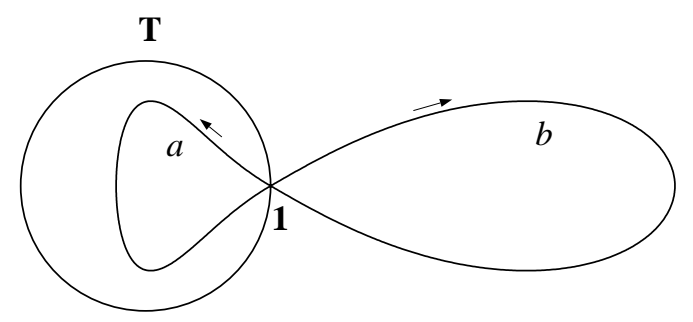

FigURE 7.

which, by $x^{2}+y^{2}=1$, has solutions $u=x+1$ and $u=-x+3$. These correspond to $\zeta=\kappa+1$ and $\zeta=-\bar{\kappa}+3$. By (4.2), the choice of $\zeta=\kappa+1$ leads to $a=\kappa$ or $a=1$, which are not acceptable since we want $|a|<1$. Therefore, we are left with the only possibility

$$
\zeta=-\bar{\kappa}+3 .
$$

Let $\kappa=e^{2 \pi i t}$ with $t \in \mathbb{R}$. From (4.2) and (4.5) it follows that $a$ and $b$ are the solutions of the quadratic equation

$$
z^{2}+(\bar{\kappa}-3) z+\kappa=0 .
$$

Lemma 4.2. As $\kappa=e^{2 \pi i t}$ goes around the unit circle, the two solutions of the quadratic equation (4.6) define two closed curves $t \mapsto a(t)$ and $t \mapsto b(t)$ in the complex plane with the following properties (see Figure 7):

(i) $a(t+1)=a(t)$ and $b(t+1)=b(t)$,

(ii) $0<|a(t)| \leq 1$ and hence $|b(t)|=|a(t)|^{-1} \geq 1$,

(iii) $|a(t)|=1$ if and only if $t \in \mathbb{Z}$, or equivalently $\kappa=1$, in which case $a(t)=$ $b(t)=1$, and

(iv) $a(t) \overline{b(t)} \neq 1$ unless $t \in \mathbb{Z}$ so that $a(t)=b(t)=1$.

Proof. Let us first note that the solutions $z_{1}, z_{2}$ of (4.6) lie on the unit circle $\mathbb{T}$ if and only if $\kappa=1$ in which case there is a double root at $z_{1}=z_{2}=1$. In fact, if $\left|z_{1}\right|=\left|z_{2}\right|=1$, then

$$
2=3-|\bar{\kappa}| \leq|\bar{\kappa}-3|=\left|z_{1}+z_{2}\right| \leq\left|z_{1}\right|+\left|z_{2}\right|=2 .
$$

Hence $|\bar{\kappa}-3|=2$, or equivalently, $\kappa=1$.

Now let $\kappa=e^{2 \pi i t}$ go around $\mathbb{T}$. Then the double root at $z=1$ splits into distinct roots $a=a(t)$ and $b=b(t)$ which by inspecting the explicit formula for $a$ and $b$ are real-analytic functions of $t$ away from integer values and are labeled so that (ii) holds. Clearly $a$ and $b$ are $\mathbb{Z}$-periodic, so (i) holds trivially.

Finally, suppose that for some $t \in \mathbb{R}, a=a(t)$ and $b=b(t)$ satisfy $a \bar{b}=1$. Then $a / \bar{a}=\kappa$, or $\bar{a}=a \bar{\kappa}$. Since $a$ is a solution of (4.6), we have

$$
\bar{a}^{2}+(\kappa-3) \bar{a}+\bar{\kappa}=0 \Longrightarrow a^{2} \bar{\kappa}^{2}+(\kappa-3) a \bar{\kappa}+\bar{\kappa}=0,
$$

or, after multiplying by $\kappa^{2}$,

$$
a^{2}+\kappa(\kappa-3) a+\kappa=0 .
$$


Comparing (4.7) and (4.6) for $z=a$, we conclude that

$$
\kappa(\kappa-3)=\bar{\kappa}-3 \Longrightarrow \kappa^{2}(\kappa-3)=1-3 \kappa \Longrightarrow(\kappa-1)^{3}=0
$$

which shows $\kappa=1$.

Lemma 4.3. For any $z \in \mathbb{T}$, the closed curve $\Gamma_{z}:[0,1] \rightarrow \mathbb{T}$ defined by

$$
\Gamma_{z}(t)=\left(\frac{z-a(t)}{1-\overline{a(t)} z}\right)\left(\frac{z-b(t)}{1-\overline{b(t)} z}\right)
$$

is null-homotopic.

Note that when $z=1$, there is no ambiguity in the definition of $\Gamma_{z}$. In fact, by (4.2) and (4.5),

$$
\Gamma_{1}=\frac{1-\zeta+\kappa}{1-\bar{\zeta}+\bar{\kappa}}=\frac{-2+\kappa+\bar{\kappa}}{-2+\kappa+\bar{\kappa}} \equiv 1
$$

so that $\Gamma_{1}$ is the constant loop 1.

Proof. Consider the two homotopies $(t, s) \mapsto a(t, s)$ and $(t, s) \mapsto b(t, s)$ rel $\{1\}$ defined by

$$
a(t, s)=(1-s) a(t)+s, \quad b(t, s)=(1-s) b(t)+s .
$$

It is easy to see that $|a(t, s)| \leq 1$ and $|b(t, s)| \geq 1$, with the equality if and only if $a(t, s)=1$ and $b(t, s)=1$. Consider the map defined by

$$
H(t, s)=\left(\frac{z-a(t, s)}{1-\overline{a(t, s)} z}\right)\left(\frac{z-b(t, s)}{1-\overline{b(t, s) z}}\right) .
$$

A brief computation shows that when $z=1, H(t, s) \equiv 1$. Evidently $H$ defines a homotopy between $H(\cdot, 0)=\Gamma_{z}$ and the constant loop $H(\cdot, 1)=1$.

Proof of Theorem 4.1. Start with the closed curves $t \mapsto a(t)$ and $t \mapsto b(t)$ of Lemma 4.2 and form the Blaschke product

$$
B^{t}: z \mapsto e^{-2 \pi i(\nu+t)} z\left(\frac{z-a(t)}{1-\overline{a(t)} z}\right)\left(\frac{z-b(t)}{1-\overline{b(t)} z}\right) .
$$

When $t$ is not an integer, $B^{t}$ has degree 3 by Lemma4.2(iv) and satisfies conditions (i) and (ii) required by Theorem 4.1 Moreover, it maps the unit circle $\mathbb{T}$ to itself, and has no critical points in $\mathbb{T}$ other than 1 , hence $\left.B^{t}\right|_{\mathbb{T}}$ is a critical circle map. So to finish the proof, it suffices to show that for some $t \notin \mathbb{Z}$, the rotation number of the restriction of $B^{t}$ to the circle $\mathbb{T}$ is equal to $\theta$. To this end, consider the universal covering map $\mathbb{R} \rightarrow \mathbb{T}$ given by $z=z(w)=e^{2 \pi i w}$. Since $B^{0}: z \mapsto e^{-2 \pi i \nu} z$, a lifting of $B^{0}$ to the real line will be the affine map $\hat{B}^{0}: w \mapsto-\nu+w$. The loop $\left\{t \mapsto B^{t}\right\}_{0 \leq t \leq 1}$ can then be lifted to a path $\left\{t \mapsto \hat{B}^{t}\right\}_{0 \leq t \leq 1}$ so that

$$
\hat{B}^{t}: w \mapsto-\nu-t+w+\frac{1}{2 \pi i} \log \left(\Gamma_{e^{2 \pi i w}}(t)\right),
$$

where $\Gamma_{z}$ is the closed curve defined in (4.8) and the branch of logarithm sends 1 to 0 . Let $\rho(t)=\lim _{n \rightarrow \infty}\left(\hat{B}^{t}\right)^{\circ n}(w) / n$. It is a standard fact that $\rho$ is well defined and independent of $w$ and the map $t \mapsto \rho(t)$ is continuous (see for example $\mathrm{dMvS}$ ). The rotation number of $\left.B^{t}\right|_{\mathbb{T}}$ is then the fractional part of $\rho(t)$. Evidently $\rho(0)=-\nu$. Since $\Gamma_{z}$ is null-homotopic by Lemma 4.3, we have $\hat{B}^{1}: w \mapsto-\nu-1+w$, so that 


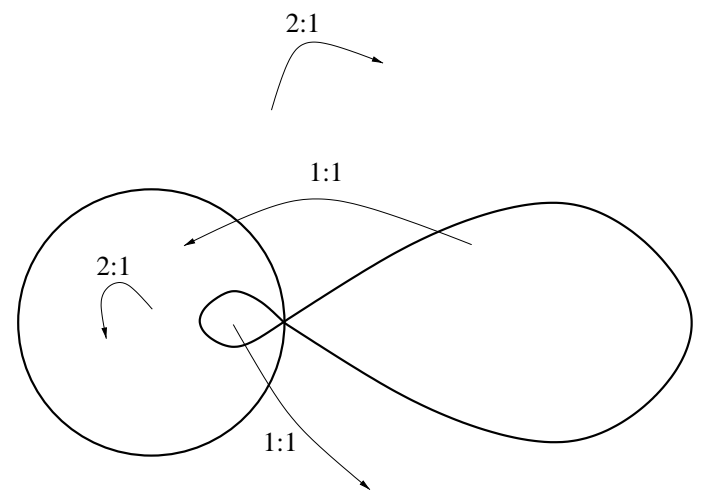

Figure 8 . The preimage $B^{-1}(\mathbb{T})$ and the basic dynamics of $B$.

$\rho(1)=-\nu-1$. Since $\theta \neq 1-\nu$, it follows that for some $0<t<1, \rho(t) \equiv \theta(\bmod$ $1)$. Hence the rotation number of the corresponding $B^{t}$ is $\theta$.

4.2. Corollaries of the construction. As we shall see below, the Blaschke product $B_{\theta, \nu}$ we constructed above and the Blaschke model $Q_{\theta}$ of $\$ 3$ share many common properties. This will allow us to define drops, limbs, drop-chains, etc. in a similar fashion for $B_{\theta, \nu}$. We will also describe a quasiconformal surgery transforming $B_{\theta, \nu}$ into the quadratic rational map $F_{\theta, \nu}$.

Let $0<\theta<1$ be irrational and $0<\nu<1$ be irrational of Brjuno type, and set $B=B_{\theta, \nu}$. By (4.1), $B(z)=e^{-2 \pi i \nu} z+O\left(z^{2}\right)$ near $z=0$, so by the theorem of Brjuno-Yoccoz [Yo2] the origin is the center of a Siegel disk $U^{0}$ for $B$. We have $U^{0} \subset \mathbb{D}$ since the unit circle is a subset of the Julia set. Since $B$ commutes with the reflection $\mathcal{T}: z \mapsto 1 / \bar{z}$, there exists a Siegel disk $U^{\infty}=\mathcal{T}\left(U^{0}\right)$ centered at infinity. In the local coordinate $w=1 / z$ near infinity, the map $w \mapsto 1 / B(1 / w)$ has the form $w \mapsto e^{2 \pi i \nu} w+O\left(w^{2}\right)$, so the rotation number of $U^{\infty}$ is $\frac{1}{2 \pi i} \log B^{\prime}(\infty)=\nu$.

$B$ has zeros at $\{0, a, b\}$ and poles at $\{\infty, 1 / \bar{a}, 1 / \bar{b}\}$. The preimage $B^{-1}(\mathbb{T})$ consists of $\mathbb{T}$ and an analytic closed curve homeomorphic to a figure eight with the double point at $z=1$. This curve and the basic dynamics of $B$ are shown in Figure 8 By the theorem of Yoccoz (see subsection 2.3), there exists a homeomorphism $h: \mathbb{T} \rightarrow \mathbb{T}$, unique if we require that $h(1)=1$, such that $\left.h \circ B\right|_{\mathbb{T}}=\varrho_{\theta} \circ h$. Denoting by $H: \mathbb{D} \rightarrow \mathbb{D}$ the Douady-Earle extension of $h$, we define the modified map $\tilde{B}$ as

$$
\tilde{B}(z)=\tilde{B}_{\theta, \nu}(z)= \begin{cases}B(z), & |z| \geq 1 \\ \left(H^{-1} \circ \varrho_{\theta} \circ H\right)(z), & |z| \leq 1\end{cases}
$$

The map $\tilde{B}$ is a degree 2 branched covering of the sphere, holomorphic outside of $\mathbb{D}$. It has a Siegel disk $U^{\infty}$ centered at $\infty$ and a "topological Siegel disk", namely the unit disk $\mathbb{D}$, on which its action is topologically conjugate to an irrational rotation.

The definition of drops and their addresses for the map $\tilde{B}$ carries over word for word from subsection 3.2 . In particular, the unit disk $\mathbb{D}$ is the 0-drop, and its immediate preimage $U_{1}=\tilde{B}^{-1}(\mathbb{D}) \backslash \mathbb{D}$ is the 1-drop of $\tilde{B}$. As before, the root of the drop $U_{\iota_{1} \iota_{2} \ldots \iota_{k}}$ is the point $x_{\iota_{1} \iota_{2} \ldots \iota_{k}}=\partial U_{\iota_{1} \iota_{2} \ldots \iota_{k-1} \iota_{k}} \cap \partial U_{\iota_{1} \iota_{2} \ldots \iota_{k-1}}$. As in subsection 3.4. for each sequence of drops $\left\{U_{0}=\mathbb{D}, U_{\iota_{1}}, U_{\iota_{1} \iota_{2}}, \ldots\right\}$ where each $U_{\iota_{1} \ldots \iota_{k}}$ is the 


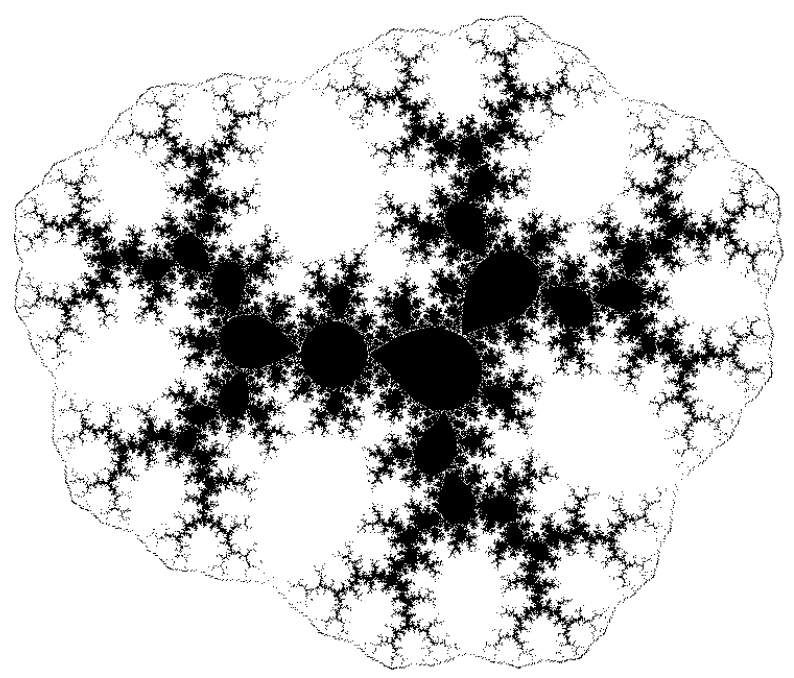

Figure 9 . Set $K\left(\tilde{B}_{\theta, \nu}\right)$ for $\theta=\nu=(\sqrt{5}-1) / 2$. Numerical experiment gives $a=-0.019048-0.298116 i, b=3.280417-0.667122 i$ for these choices of $\theta$ and $\nu$. There is a striking similarity with the corresponding picture for the quadratic rational map $F$ of Figure 2 up to a $90^{\circ}$ rotation. The reason is the existence of a quasiconformal homeomorphism conjugating $\tilde{B}_{\theta, \nu}$ to $F$ which is conformal in the white region.

parent of $U_{\iota_{1} \ldots \iota_{k+1}}$, we define the drop-chain

$$
\mathcal{C}=\overline{\bigcup_{k} U_{\iota_{1} \ldots \iota_{k}}}
$$

and the corresponding drop-ray $R(\mathcal{C}) \subset \mathcal{C}$. We can also define the limb $L_{\iota_{1} \ldots \iota_{k}}$ as the closure of the union of $U_{\iota_{1} \ldots \iota_{k}}$ and all its descendants:

$$
L_{\iota_{1} \ldots \iota_{k}}=\overline{\bigcup U_{\iota_{1} \ldots \iota_{k} \ldots}} \text {. }
$$

In anticipation of the analogue of Theorem 3.5, let us define the accumulation set of the drop-chain $\mathcal{C}$ in (4.10) as the intersection of the decreasing sequence of limbs $L_{\iota_{1}} \supset L_{\iota_{1} \iota_{2}} \supset L_{\iota_{1} \iota_{2} \iota_{3}} \supset \cdots$. When this set is a single point $\{p\}$, we say that $R(\mathcal{C})$ or $\mathcal{C}$ lands at $p$.

As an analogue to the "filled Julia set" $K(\tilde{Q})$, we define

$$
K(\tilde{B})=K\left(\tilde{B}_{\theta, \nu}\right)=\left\{z \in \mathbb{C}: \text { the orbit }\left\{\tilde{B}^{\circ n}(z)\right\}_{n \geq 0} \text { never intersects } U^{\infty}\right\}
$$

and

$$
J(\tilde{B})=\partial K(\tilde{B}) .
$$

Both sets are non-empty and compact. However, $K(\tilde{B})$ is no longer full. The simply-connected basin of infinity for $\tilde{Q}$ is replaced by the Siegel disk $U^{\infty}$ of $\tilde{B}$ and all its infinitely many preimages (compare Figure 9).

Finally, when $\theta$ is of bounded type, we can perform the same kind of quasiconformal surgery as in subsection 3.5 to obtain a quadratic rational map from $\tilde{B}$. In this case by Herman's theorem (see subsection [2.3) the homeomorphism $h$ which 
linearizes $\left.B\right|_{\mathbb{T}}$ is quasisymmetric, therefore its Douady-Earle extension $H$ is quasiconformal. The map $\tilde{B}=\tilde{B}_{\theta, \nu}$ is a quasiregular branched covering of the Riemann sphere. We define a $\tilde{B}_{\theta, \nu}$-invariant conformal structure $\sigma_{\theta, \nu}$ on the sphere by setting it equal to the standard structure $\sigma_{0}$ on $\mathbb{C} \backslash K\left(\tilde{B}_{\theta, \nu}\right)$, to $H^{*} \sigma_{0}$ on $\mathbb{D}$, and to $\left(\tilde{B}_{\theta, \nu}^{\circ n}\right)^{*} H^{*} \sigma_{0}=\left(B_{\theta, \nu}^{\circ n}\right)^{*} H^{*} \sigma_{0}$ on every drop of depth $n$. The maximal dilatation of $\sigma_{\theta, \nu}$ is equal to the dilatation of $H$, and by the Measurable Riemann Mapping Theorem there exists a quasiconformal homeomorphism $\psi: \widehat{\mathbb{C}} \rightarrow \widehat{\mathbb{C}}$ with $\psi^{*} \sigma_{0}=\sigma_{\theta, \nu}$. The conjugate map $F=\psi \circ \tilde{B}_{\theta, \nu} \circ \psi^{-1}$ is a degree 2 holomorphic branched covering of the sphere, that is, a quadratic rational map. Let us normalize $\psi$ by assuming $\psi(\infty)=\infty, \psi\left(H^{-1}(0)\right)=0$ and $\psi(\beta)=1$, where $\beta$ denotes the fixed point of $B_{\theta, \nu}$ in $\mathbb{C} \backslash\left(U^{\infty} \cup \mathbb{D}\right)$. By inspection, we have $F=F_{\theta, \nu}$ in $(2.1)$, so that

$$
F_{\theta, \nu}=\psi \circ \tilde{B}_{\theta, \nu} \circ \psi^{-1} \text {. }
$$

Recall that $F_{\theta, \nu}$ has two Siegel disks $\Delta^{0}$ and $\Delta^{\infty}$ centered at 0 and $\infty$, which satisfy $\Delta^{0}=\psi(\mathbb{D})$ and $\Delta^{\infty}=\psi\left(U^{\infty}\right)$. As a first consequence we obtain

Theorem 4.1. Let $0<\theta<1$ be an irrational of bounded type. Then the boundary of the Siegel disk $\Delta^{0}$ of $F_{\theta, \nu}$ is a quasicircle passing through a single critical point of $F_{\theta, \nu}$.

Observe that there is a natural symmetry

$$
F_{\theta, \nu}=\mathcal{I} \circ F_{\nu, \theta} \circ \mathcal{I}
$$

where $\mathcal{I}$ is the involution $z \mapsto 1 / z$.

Corollary 4.4. Suppose that both $0<\theta<1$ and $0<\nu<1$ are irrationals of bounded type. Then the boundaries of the Siegel disks $\Delta^{0}$ and $\Delta^{\infty}$ of $F_{\theta, \nu}$ are disjoint quasicircles, each passing through a critical point of $F_{\theta, \nu}$.

The involution $\mathcal{I}$ provides us with a quasiconformal conjugacy between $\tilde{B}_{\theta, \nu}$ and $\tilde{B}_{\nu, \theta}$. In particular, setting

$$
K^{\infty}\left(\tilde{B}_{\theta, \nu}\right)=\overline{\widehat{\mathbb{C}} \backslash K\left(\tilde{B}_{\theta, \nu}\right)},
$$

we have

Corollary 4.5. There exists a quasiconformal homeomorphism of the Riemann sphere mapping the set $K^{\infty}\left(\tilde{B}_{\theta, \nu}\right)$ to $K\left(\tilde{B}_{\nu, \theta}\right)$.

Hence for the map $\tilde{B}_{\theta, \nu}$ we can naturally define the drops growing from infinity $U_{\iota_{1} \ldots \iota_{k}}^{\infty} \subset \widehat{\mathbb{C}} \backslash K\left(\tilde{B}_{\theta, \nu}\right)$, with $U_{0}^{\infty}=U^{\infty}$, limbs growing from infinity $L_{\iota_{1} \ldots \iota_{k}}^{\infty}$, etc.

We conclude with another immediate corollary of the above construction:

Corollary 4.6. With the above notation, $\partial K\left(\tilde{B}_{\theta, \nu}\right)=\partial K^{\infty}\left(\tilde{B}_{\theta, \nu}\right)$.

Proof. Under the surgery construction, both sets $\partial K\left(\tilde{B}_{\theta, \nu}\right)$ and $\partial K^{\infty}\left(\tilde{B}_{\theta, \nu}\right)$ correspond to the Julia set $J\left(F_{\theta, \nu}\right)$.

\section{Construction of PuZzle-Pieces}

The goal of this section and the next one is to establish the following analogue of Theorem 3.5 . 
Theorem 5.1. Let $0<\theta, \nu<1$ be irrationals of bounded type, with $\theta \neq 1-\nu$, and consider the modified Blaschke product $\tilde{B}_{\theta, \nu}$ of (4.9). Then as the depth of a limb $L_{\iota_{1} \ldots \iota_{k}}$ goes to infinity, $\operatorname{diam}\left(L_{\iota_{1} \ldots \iota_{k}}\right)$ goes to zero.

It follows from Corollary 4.5 that $\operatorname{diam}\left(L_{\iota_{1} \ldots \iota_{k}}^{\infty}\right) \rightarrow 0$ as $\iota_{1}+\ldots+\iota_{k} \rightarrow \infty$.

We start by constructing puzzle-pieces. Our construction closely parallels the one presented by Petersen in Pe]. For simplicity, set $B=B_{\theta, \nu}$ and $\tilde{B}=\tilde{B}_{\theta, \nu}$. Denote by $\mathcal{C}$ the drop-chain

$$
\mathcal{C}=\overline{U_{0} \cup U_{1} \cup U_{11} \cup U_{111} \cup \cdots} .
$$

The following refinement of the Douady-Hubbard-Sullivan landing theorem can be found in $[\mathrm{TY}$ :

Lemma 5.2. Let $F$ be a rational map and let $\Lambda$ denote the closure of the union of the postcritical set and possible rotation domains of $F$. Suppose that $n \geq 1$ and $\gamma:(-\infty, 0] \rightarrow \widehat{\mathbb{C}} \backslash \Lambda$ is a curve with

$$
F^{\circ n k}(\gamma(-\infty,-k])=\gamma(-\infty, 0]
$$

for all positive integers $k$. Then $\lim _{t \rightarrow-\infty} \gamma(t)$ exists and is a repelling or parabolic periodic point of $F$ whose period divides $n$.

We can apply the above lemma to the drop-chain $\mathcal{C}$, setting $\gamma$ to be the drop-ray $R(\mathcal{C})$ parametrized so that the root of the $(k+1)$-st drop corresponds to $t=-k$. We conclude that $R(\mathcal{C})$ lands at the unique fixed point $\beta$ of $B$ in $\widehat{\mathbb{C}} \backslash\left(\overline{\mathbb{D} \cup U^{\infty}}\right)$. Since $\beta$ is necessarily repelling, the drops in $\mathcal{C}$ decrease geometrically in size, and the drop-chain $\mathcal{C}$ lands at the point $\beta$. Repeating the argument, we see that the drop-ray $R(\mathcal{D})$ associated to the drop-chain

$$
\mathcal{D}=\overline{U^{\infty} \cup U_{1}^{\infty} \cup U_{11}^{\infty} \cup U_{111}^{\infty} \cup \cdots}
$$

lands at a fixed point as well, which is necessarily $\beta$. Let $\mathcal{C}^{\prime}$ be the drop-chain $\overline{U_{0} \cup U_{2} \cup U_{21} \cup \cdots}$ mapped to $\mathcal{C}$ by $\tilde{B}$, and similarly define the drop-chain $\mathcal{D}^{\prime}=$ $\overline{U^{\infty} \cup U_{2}^{\infty} \cup U_{21}^{\infty} \cup \cdots}$. Then $\mathcal{C}^{\prime}$ and $\mathcal{D}^{\prime}$ have a common landing point $\beta^{\prime} \neq \beta$, which is a preimage of $\beta$ in $\widehat{\mathbb{C}} \backslash\left(\overline{\mathbb{D} \cup U^{\infty}}\right)$.

As before, the moments of closest returns of the critical point $z=1$ are denoted by $\left\{q_{n}\right\}$. Recall that these numbers appear as the denominators of the convergents of the continued fraction of $\theta$. We define the 0 -th critical puzzle-piece $P_{0}$ as the closure of the connected component of

$$
\widehat{\mathbb{C}} \backslash\left(\mathcal{C} \cup \mathcal{C}^{\prime} \cup \mathcal{D} \cup \mathcal{D}^{\prime}\right)
$$

which contains the arc $\left[1, B^{-1}(1)\right] \ni B^{\circ q_{1}}(1)$ in the boundary (see Figure 10). We inductively define the $n$-th critical puzzle-piece $P_{n} \subset \mathbb{C} \backslash \mathbb{D}$ as the closed set which is mapped homeomorphically onto $P_{n-1}$ by $B^{\circ q_{n}}$ and which contains the arc $\left[1, B^{-q_{n}}(1)\right] \subset \mathbb{T}$ in the boundary. The following proposition summarizes some of the properties of critical puzzle-pieces:

Proposition 5.3 (Properties of puzzle-pieces). (i) Each puzzle-piece $P_{n}$ is a closed Jordan domain in $\mathbb{C} \backslash \mathbb{D}$ which intersects the unit circle $\mathbb{T}$ along the $\operatorname{arc}\left[1, B^{-q_{n}}(1)\right]$.

(ii) $B^{\circ q_{n}}\left(P_{n} \cap \partial U_{1}\right)=\left[B^{\circ q_{n}}(1), B^{-q_{n-1}}(1)\right]$.

(iii) $B^{\circ q_{n}+q_{n-1}+q_{n-2}}\left(P_{n} \cap \partial U_{q_{n}+1}\right)=\left[1, B^{\circ q_{n-1}+q_{n-2}}(1)\right]$.

(iv) $P_{n}$ contains the drop $U_{q_{n+2}+1}$. 


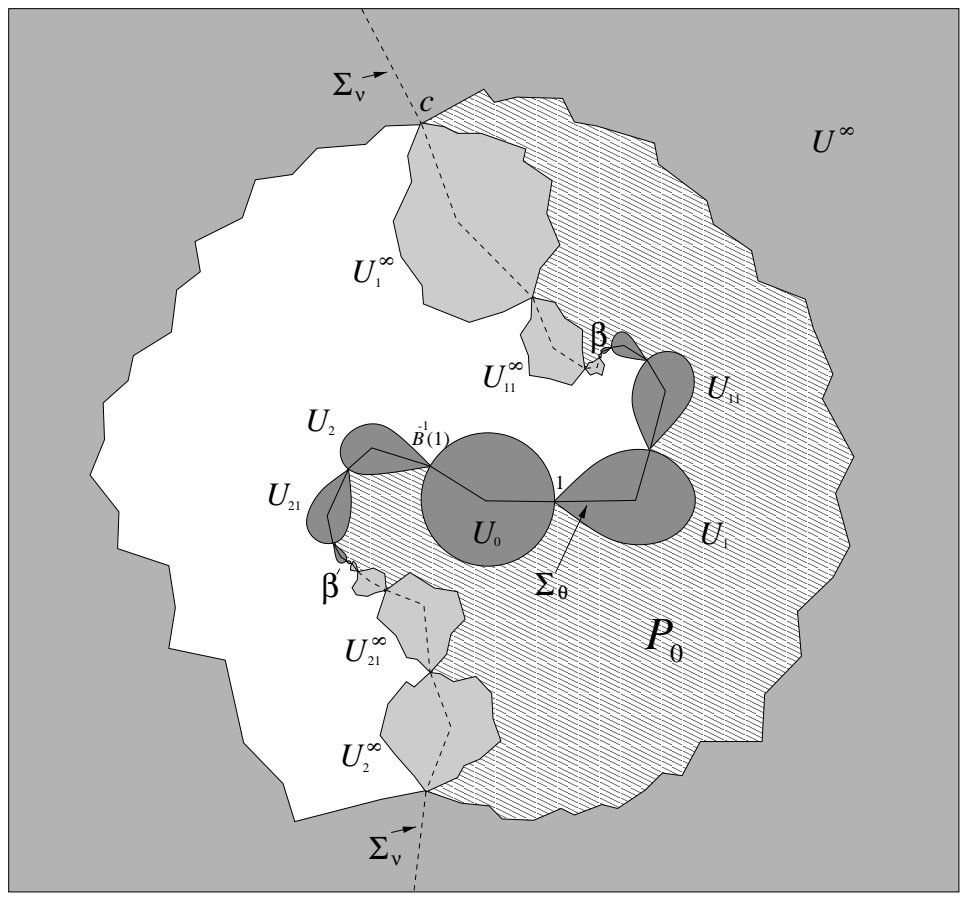

Figure 10 . The 0 -th critical puzzle-piece $P_{0}$ and the "spines" $\Sigma_{\theta}$ and $\Sigma_{\nu}$ (see §]7).

Proof. Observe that $B^{\circ q_{n}}$ is a homeomorphism $\left[B^{-q_{n}}(1), B^{-q_{n}-q_{n-1}}(1)\right] \stackrel{\simeq}{\longrightarrow}$ $\left[B^{-q_{n-1}}, 1\right]$ with one critical point at 1 . Thus the univalent inverse branch $B^{-q_{n}}$ sending $P_{n-1}$ to $P_{n}$ maps the arc $\left[B^{-q_{n-1}}, 1\right]$ onto the union of $\left[1, B^{-q_{n}}(1)\right]$ and a subarc of $\partial U_{1}$. The first three statements now follow by induction on $n$. As seen from the combinatorics of closest returns (see subsection 2.3), $\partial U_{q_{n+2}+1} \cap \mathbb{T}=$ $B^{-q_{n+2}}(1)$ is contained in the arc $\left[1, B^{-q_{n}}(1)\right]$. Evidently, the drop $U_{q_{n+2}+1}$ has no intersections with $\partial P_{n}$, so we have $U_{q_{n+2}+1} \subset P_{n}$.

In what follows, to obtain a univalent preimage of the puzzle-piece $P_{0}$, we always use holomorphic inverse branches of $\tilde{B}$. These preimages have the following nesting property:

Lemma 5.4. Let $A_{1}$ and $A_{2}$ be two distinct univalent preimages of the puzzle-piece $P_{0}$ such that $\stackrel{\circ}{A}_{1} \cap \stackrel{\circ}{A}_{2} \neq \emptyset$. Then either $A_{1} \subset A_{2}$ or $A_{2} \subset A_{1}$.

Proof. By construction, the boundary of the puzzle-piece $P_{0}$ consists of an open arc $\gamma \subset \mathcal{C} \cup \mathcal{C}^{\prime}$ which is made up of the boundary arcs of various drops $U_{\iota_{1} \ldots \iota_{k}}$, a similarly defined $\operatorname{arc} \gamma^{\infty} \subset \mathcal{D} \cup \mathcal{D}^{\prime}$ and points $\beta, \beta^{\prime}$ (see Figure 10). Denote by $\gamma_{1}$, $\gamma_{1}^{\infty}, \beta_{1}, \beta_{1}^{\prime}$ the corresponding parts of $\partial A_{1}$, and label the boundary of $A_{2}$ in the same way.

Evidently $\gamma_{1}$ does not intersect $\gamma_{2}^{\infty}$ or the points $\beta_{2}, \beta_{2}^{\prime}$, so it can only intersect $\gamma_{2}$. Similarly, $\gamma_{1}^{\infty}$ can only intersect $\gamma_{2}^{\infty}$. If $y \in\left\{\beta_{1}, \beta_{1}^{\prime}\right\} \cap\left\{\beta_{2}, \beta_{2}^{\prime}\right\}$, then $B^{-k}(\beta)=y$ for some choice of the inverse branch. Since $\beta$ is not in the post-critical set of $B$, this branch of $B^{-k}$ has a univalent extension to a neighborhood of $\beta$ intersecting 
the boundary of $P_{0}$ along a non-empty open arc. Pulling back, it follows that for some neighborhood $D$ of $y, \gamma_{1} \cap D=\gamma_{2} \cap D$ and $\gamma_{1}^{\infty} \cap D=\gamma_{2}^{\infty} \cap D$. In particular, if $\beta_{1}=\beta_{1}^{\prime}$ and $\beta_{2}=\beta_{2}^{\prime}$, we must have $A_{1}=A_{2}$.

Now assume that the lemma is false. Let $A_{1}=B^{-m}\left(P_{0}\right)$ and $A_{2}=B^{-n}\left(P_{0}\right)$, with $m \leq n$. Then by the above observation, either $\gamma_{2}$ or $\gamma_{2}^{\infty}$ must intersect both $\AA_{1}$ and $\mathbb{C} \backslash A_{1}$. Therefore, either $B^{\circ m}\left(\gamma_{2}\right)$ or $B^{\circ m}\left(\gamma_{2}^{\infty}\right)$ must intersect both $\stackrel{\circ}{P}_{0}$ and $\mathbb{C} \backslash P_{0}$. To fix the ideas, let us assume that $B^{\circ m}\left(\gamma_{2}\right)$ does. Note that $B^{\circ m}\left(\gamma_{2}\right) \cap \partial P_{0} \subset \gamma$, hence $B^{\circ m}\left(\gamma_{2}\right)$ should cross $\gamma$ at a root $x$ of a drop $U$ in $\mathcal{C} \cup \mathcal{C}^{\prime}$. Consider a small open arc $\delta \subset B^{\circ m}\left(\gamma_{2}\right) \cap \partial U$ which contains $x$. Note that the orbit segment $\left\{x, B(x), \ldots, B^{\circ n-m}(x)\right\}$ does not contain the critical point 1 since otherwise $1 \in B^{\circ j}(\delta) \subset \mathbb{T}$ for some $0 \leq j \leq n-m$, which contradicts the fact that $B^{\circ j+m}\left(A_{2}\right)$ is a univalent preimage of $P_{0}$. Now $B^{\circ n-m}$ maps $\delta$ homeomorphically to the subarc $B^{\circ n-m}(\delta) \subset \gamma$ crossing $\gamma$ at $B^{\circ n-m}(x)$, which is clearly impossible.

Corollary 5.5. For all $n \geq 0$ we have $P_{n+2} \subsetneq P_{n}$.

Proof. It is clear from the definition of critical puzzle-pieces that $\stackrel{\circ}{P}_{n+2} \cap \stackrel{\circ}{P}_{n} \neq \emptyset$. By Proposition 5.3(i), $P_{n+2} \cap \mathbb{T} \subsetneq P_{n} \cap \mathbb{T}$. The claim now follows from Lemma5.4

Lemma 5.6. Let $U$ be a topological disk whose boundary is contained in a finite union of the boundary arcs of drops (resp. drops growing from infinity). Then $U$ itself must be a drop (resp. drop growing from infinity).

Proof. Let us consider the case of drops. The proof for the case of drops growing from infinity is similar. It is easy to check that $U$ cannot contain $U_{1}^{\infty}$ or any of its preimages. Since the Blaschke product $B$ satisfies the Maximum Principle in $\mathbb{C} \backslash U_{1}^{\infty}$ and $B^{\circ n}(\partial U) \subset \mathbb{T}$ for a large $n$, we must have $B^{\circ n}(U) \subset \mathbb{D}$, which means $U$ itself is a drop.

Lemma 5.7. Let $A$ be a univalent preimage of the puzzle-piece $P_{0}$. Suppose that a drop at infinity $U_{\iota_{1} \ldots \iota_{k}}^{\infty}$ is contained in $A$. Then $A$ contains the entire limb $L_{\iota_{1} \ldots \iota_{k}}^{\infty}$.

Proof. Let us denote by $\gamma_{A}^{\infty} \subset \partial A$ the subarc of the boundary of $A$ which is made up of the boundary arcs of drops at infinity. Assume by way of contradiction that there is a drop at infinity $U_{\iota_{1} \ldots \iota_{k} \ldots \iota_{k+m}}^{\infty} \not \subset A$. Let $\mathcal{D}$ be a drop-chain containing $U_{\iota_{1} \ldots \iota_{k} \ldots \iota_{k+m}}^{\infty}$. Let $\delta \subset \partial \mathcal{D}$ be an arc connecting the root of $U_{\iota_{1}}^{\infty}$ to a point in $\partial U_{\iota_{1} \ldots \iota_{k} \ldots \iota_{k+m}}^{\infty} \backslash \bar{A}$. Then $\delta$ goes in and out of $A$, but it intersects $\partial A$ only at the points of $\gamma_{A}^{\infty}$. Thus the curves $\delta$ and $\gamma_{A}^{\infty}$ bound a topological disk $U \subset \stackrel{\circ}{A}$. By Lemma 5.6, $U$ itself is a drop growing from infinity. Since $U$ shares a non-trivial boundary arc with another drop growing from infinity, we arrive at a contradiction.

Lemma 5.8. There exists a constant $K>1$ depending only on the map $B$ such that the following holds. For a critical puzzle-piece $P_{n}$, let $b_{n}$ be its reflection $\mathcal{T}\left(P_{n}\right)$ through the unit circle. Then the union $P_{n} \cup b_{n}$ contains a Euclidean disk $D$ centered at a point in $\mathbb{T}$ whose diameter is $K$-commensurable with $\left|\left[1, B^{-q_{n}}(1)\right]\right|$.

Proof. It suffices to find such $K$ which works for all sufficiently large $n$, for then, by making $K$ larger if necessary, we will have the result for all $n$. As usual, in what follows "commensurable" means $C$-commensurable for some $C>1$ independent of $n$. For $n \geq 2$, set $s_{n}=q_{2}+\cdots+q_{n}$. By definition of critical puzzle-pieces, 
$B^{\circ s_{n}}\left(P_{n}\right)=P_{1}$, and by symmetry $B^{\circ s_{n}}\left(b_{n}\right)=b_{1}$. Let $\psi$ be the univalent branch of $B^{-s_{n}}$ mapping $\stackrel{\circ}{P}_{1} \cup \stackrel{\circ}{b}_{1}$ to $\stackrel{\circ}{P}_{n} \cup \stackrel{\circ}{b}_{n}$. By the combinatorics of closest returns, $\psi$ maps $\left(B^{-\left(3 q_{n+4}-s_{n}\right)}(1), B^{\circ s_{n}}(1)\right) \subset\left[B^{-q_{1}}(1), 1\right]=P_{1} \cap \mathbb{T}$ diffeomorphically to $\left(B^{-3 q_{n+4}}(1), 1\right) \subset\left[B^{-q_{n}}(1), 1\right]=P_{n} \cap \mathbb{T}$. By Światek-Herman real a priori bounds (see subsection 2.3), the interval $E_{n}=\left[B^{-\left(2 q_{n+4}-s_{n}\right)}(1), B^{-\left(q_{n+4}-s_{n}\right)}(1)\right]$ is commensurable with and well inside of $E_{n}^{\prime}=\left(B^{-\left(3 q_{n+4}-s_{n}\right)}(1), B^{\circ s_{n}}(1)\right)$. For large $n$, the closed Euclidean disk $D_{1}$ centered on $\mathbb{T}$ which intersects $\mathbb{T}$ along $E_{n}$ is contained in the open topological disk $A=\stackrel{\circ}{P}_{1} \cup \stackrel{\circ}{b}_{1} \cup E_{n}^{\prime}$ in such a way that the topological annulus $A \backslash D_{1}$ has definite modulus independent of $n$. By the Koebe distortion theorem, the distortion of $\psi$ restricted to $D_{1}$ has a bound independent of $n$, and hence $\psi\left(D_{1}\right)$ is an almost round disk in $P_{n} \cup b_{n}$ whose diameter is commensurable with $\psi\left(E_{n}\right)=\left[B^{-2 q_{n+4}}(1), B^{-q_{n+4}}(1)\right]$. By real a priori bounds, this interval is commensurable with $\left[B^{-q_{n+4}}(1), 1\right]$, which is in turn commensurable with $\left[B^{-q_{n}}(1), 1\right]$. Now the largest disk $D$ contained in $\psi\left(D_{1}\right)$ and centered on $\mathbb{T}$ has the desired properties.

The last property of puzzle-pieces we need is the following:

Lemma 5.9. There exists $N>0$ such that for all $n \geq N$ the critical puzzle-piece $P_{n}$ does not intersect $\partial U^{\infty}$.

Proof. Since the boundary of the Siegel disk $U^{\infty}$ is forward-invariant, we only need to show the existence of one $N$ such that $P_{N} \cap \partial U^{\infty}=\emptyset$. Assume this is false. Let us denote by $\gamma_{n}$ an arc in $\partial P_{n}$ connecting 1 to $\partial U^{\infty}$. By Lemma 5.4 the curves in the orbit

$$
\gamma_{n}, B\left(\gamma_{n}\right), \ldots, B^{\circ q_{n}-1}\left(\gamma_{n}\right)
$$

are disjoint. By the theorem of Yoccoz (see subsection 2.3) the maps $\left.B\right|_{\mathbb{T}}$ and $\left.B\right|_{\partial U^{\infty}}$ are topologically conjugate to rigid rotations. Since the orbit of a point under an irrational rotation is dense on the circle, the maximum diameter of the pieces into which the curves 5.1 partition the boundaries of $\mathbb{D}$ and $U^{\infty}$ goes to zero as $n \rightarrow \infty$. We may therefore construct an orientation-reversing topological conjugacy between the circle maps $\left.B\right|_{\mathbb{T}}$ and $\left.B\right|_{\partial U_{\infty}}$. This contradicts the fact that $\theta \neq 1-\nu$.

\section{Complex Bounds}

The proof of Petersen's Theorem presented in $\mathrm{Ya}$ is based on a version of estimates employed in the same paper for proving a renormalization convergence result. In renormalization theory it is customary to use the term complex a priori bounds for such estimates. Our goal in this section is to adapt these bounds to the Blaschke product model introduced in $\$ 4$

As before, let us fix irrationals $0<\theta, \nu<1$ of bounded type, with $\theta \neq 1-\nu$, and set $B=B_{\theta, \nu}, \tilde{B}=\tilde{B}_{\theta, \nu}$. Recall that $B$ is a Blaschke product of the form

$$
B=z \mapsto \lambda z\left(\frac{z-a}{1-\bar{a} z}\right)\left(\frac{z-b}{1-\bar{b} z}\right),
$$

where $|\lambda|=1,0<|a|<1$ and $|b|=|a|^{-1}$. We set

$$
B(1)=e^{2 \pi i \tau} \quad \text { with } 0<\tau<1 \text {. }
$$




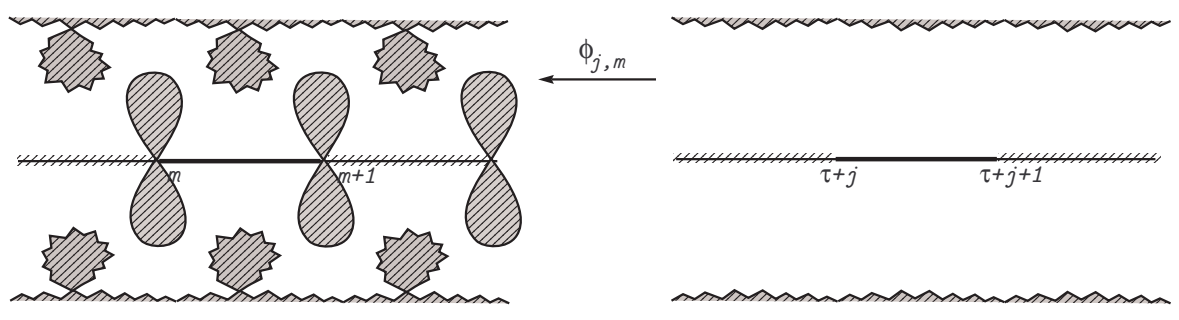

Figure 11.

The convergents of the continued fraction $\theta=\left[a_{1}, a_{2}, a_{3}, \ldots\right]$ will be denoted $\left\{p_{n} / q_{n}\right\}$. Observe that $(B(z)-B(1)) /(z-1)^{3}$ is a bounded holomorphic function in the domain $\mathbb{C} \backslash \overline{\left(\mathbb{D} \cup U^{\infty} \cup U_{1}^{\infty}\right)}$. Consequently,

$$
C^{-1}|z-1|^{3}<|B(z)-B(1)|<C|z-1|^{3}
$$

in this domain, for some positive constant $C$.

Recall that $U^{0}$ and $U^{\infty}$ denote Siegel disks of $B$ centered at 0 and $\infty$. Let $S$ be the translation-invariant infinite strip which is mapped onto the open topological annulus $\mathbb{C} \backslash\left(\overline{U^{0}} \cup \overline{U^{\infty}}\right)$ by the exponential map $z \mapsto e^{2 \pi i z}$. Let us denote by $S_{J}$ the domain obtained by removing from $S$ the points of the real line that do not belong to the open interval $J \subset \mathbb{R}$ :

$$
S_{J}=(S \backslash \mathbb{R}) \cup J .
$$

Let $\hat{B}(z)$ denote the (multi-valued) meromorphic function $\frac{1}{2 \pi i} \log B\left(e^{2 \pi i z}\right)$ on $S$. On the real line $\hat{B}$ has singularities at the integer points, whose images lie at the integer translates of $0<\tau<1$. Other singularities of $\hat{B}$ lie at the boundary curves of $S$ at the points $\pm s+j, j \in \mathbb{Z}$, which are mapped by the exponential map to the critical points on the boundaries of the Siegel disks $U^{0}$ and $U^{\infty}$. By the Monodromy Theorem, in the domain $S_{(\tau+j, \tau+j+1)}$ with the critical values removed, we have welldefined branches $\phi_{j, m}$ of the inverse $\hat{B}^{-1}$, mapping the open interval $(\tau+j, \tau+j+1)$ homeomorphically onto the interval between two consecutive integers $(m, m+1)$ (see Figure 11). The range of the map $\phi_{j, m}$ is the simply-connected region

$$
S_{(m, m+1)} \backslash\left[\left(\frac{ \pm 1}{2 \pi i} \log \left(\overline{U_{1}}\right)\right) \cup\left(\frac{ \pm 1}{2 \pi i} \log \left(\overline{U_{1}^{\infty}}\right)\right)\right] .
$$

Denote by $\Upsilon: \mathbb{T} \backslash\{B(1)\} \rightarrow I=(\tau-1, \tau)$ the single-valued branch of $\frac{1}{2 \pi i} \log (z)$ mapping 1 to 0 . Define the (interval exchange like) map $\phi: I \rightarrow I$ by

$$
\phi(z)= \begin{cases}\phi_{-1,0}(z) & \text { for } z \in\left(\tau-1, \Upsilon\left(B^{\circ 2}(1)\right)\right] \\ \phi_{-1,-1}(z) & \text { for } z \in\left(\Upsilon\left(B^{\circ 2}(1)\right), \tau\right) .\end{cases}
$$

Let us fix an $n \geq 2$ and consider the backward orbit of open intervals

$$
\left(1, B^{\circ q_{n}}(1)\right),\left(B^{-1}(1), B^{\circ q_{n}-1}(1)\right), \ldots,\left(B^{-q_{n}}(1), 1\right) .
$$

Set $J_{-i}=\Upsilon\left(\left(B^{-i}(1), B^{\circ q_{n}-i}(1)\right)\right)$ and consider the $\phi$-orbit

$$
J_{0}, J_{-1}, J_{-2}, \ldots, J_{-q_{n}} \text {. }
$$


Using the combinatorics of closest returns (see subsection 2.3), it is not hard to see that $B^{\circ 2}(1) \notin\left(B^{-k}(1), B^{\circ q_{n}-k}(1)\right)$ for $0 \leq k \leq q_{n}$. In other words, the intervals of the orbit (6.4) do not contain the point of discontinuity of the map $\phi$. By its definition, the map $\phi: J_{-k} \rightarrow J_{-k-1}$ for $0 \leq k \leq q_{n}-1$ has a univalent extension to $S_{J_{-k}}$. As seen from (6.2) the range of this univalent map is a subset of $S_{J_{-k-1}}$, hence the composition $\phi^{\circ \ell}: J_{-k} \rightarrow J_{-k-\ell}$ for $0 \leq k<k+\ell \leq q_{n}$ univalently extends to the entire $S_{J_{-k}}$.

Consider the univalent extensions of the iterates $\phi^{\circ k}: J_{0} \rightarrow J_{-k}$ to the strip $S_{J_{0}}$ for $1 \leq k \leq q_{n}$. Applying these univalent branches to a point $z \in S_{J_{0}}$, we obtain the backward orbit of $z$ corresponding to the orbit (6.4):

$$
z=z_{0}, z_{-1}, z_{-2}, \ldots, z_{-q_{n}} \text {, where } z_{-k}=\phi^{\circ k}\left(z_{0}\right) \text {. }
$$

A corresponding backward orbit of a subset of $S_{J_{0}}$ is similarly defined.

Let $\mathbb{C}_{J} \supset S_{J}$ denote the slit plane $(\mathbb{C} \backslash \mathbb{R}) \cup J$. One easily constructs a conformal mapping of this domain to the upper half-plane to verify that the hyperbolic neighborhood $\left\{z \in \mathbb{C}_{J} \mid \operatorname{dist}_{\mathbb{C}_{J}}(z, J)<r\right\}$ for $r>0$ is the union $D_{\theta}(J)$ of two Euclidean disks of equal radii with common chord $J$ intersecting the real axis at an outer angle $\theta=\theta(r)$ (see $[\mathrm{dMvS}$ ). In this case, an elementary computation yields

$$
r=\log \tan \left(\frac{\pi}{2}-\frac{\theta}{4}\right) .
$$

A standard argument shows that the hyperbolic neighborhood $\left\{z \in S_{J} \mid \operatorname{dist}_{S_{J}}(z, J)\right.$ $<r\}$ also forms angles $\theta=\theta(r)$ with $\mathbb{R}$. We choose the notation $G_{\theta}(J)$ for this neighborhood. The Schwarz Lemma implies that $G_{\theta}(J) \subset D_{\theta}(J)$.

Let $\breve{S} \subset \mathbb{C}$ be a horizontal strip invariant under the unit translation, such that $\breve{S} / \mathbb{Z}$ is compactly contained in $S / \mathbb{Z}$. A specific choice of $\breve{S}$ will be made later (just before Lemma 6.5 below). For a bounded interval $I \subset \mathbb{R}$ and a point $z \in S_{I} \backslash \mathbb{R}$, denote by $0<\widehat{(z, I)}<\pi$ the least of the outer angles the segments joining $z$ to the end-points of $I$ form with the real line. The following adaptation of Lemma 2.1 of Ya will be used to control the expansion of inverse branches:

Lemma 6.1. Given $\epsilon>0$ and any strip $\breve{S}$ as above, there exists a constant $C=$ $C(\epsilon, \breve{S})>0$ such that for every point $z=z_{0} \in S_{J_{0}}$ and every pair of natural numbers $n \geq 2$ and $1 \leq k \leq q_{n}-1$ the following holds: Let $z_{0}, \ldots, z_{-q_{n}}$ be the backward orbit (6.5) and assume for some $0 \leq i \leq k$ we have $z_{-i} \in \breve{S}$ and $\left(\widehat{z_{-i}, J_{-i}}\right)>\epsilon$. Then

$$
\frac{\operatorname{dist}\left(z_{-k}, J_{-k}\right)}{\left|J_{-k}\right|} \leq C \frac{\operatorname{dist}\left(z_{-i}, J_{-i}\right)}{\left|J_{-i}\right|} .
$$

Proof. First observe that $\left.B^{-\left(q_{n}-1\right)}\right|_{\mathbb{T}}$ is a diffeomorphism on $\left[B^{\circ 2 q_{n}}(1), B^{-q_{n}}(1)\right]$, which contains $\left[B^{\circ q_{n}}(1), 1\right]$ in its interior. Moreover, by Świastek-Herman real a priori bounds (see subsection [2.3), the latter arc is well inside of the former with the bound independent of $n$. Setting $H=\Upsilon\left(\left(B^{\circ 2 q_{n}}(1), B^{-q_{n}}(1)\right)\right)$, we see that $J_{0}$ is well inside of $H$, and $\phi^{\circ j}: J_{0} \rightarrow J_{-j}$ univalently extends to $S_{H}$ for $1 \leq j \leq q_{n}-1$. Set $T=\phi^{\circ i}(H) \supset J_{-i}$. By the Koebe distortion theorem, there exists $\rho>0$ such that both components of $T \backslash J_{-i}$ have length at least $2 \rho\left|J_{-i}\right|$. Note that the iterate

$$
\phi^{\circ k-i}: J_{-i} \rightarrow J_{-k}
$$

has a univalent extension to $S_{T}$. 
Let us normalize the situation by considering the orientation-preserving affine maps

$$
\alpha_{1}: J_{-i} \rightarrow(0,1) \text { and } \alpha_{2}: J_{-k} \rightarrow(0,1)
$$

The composition $\alpha_{2} \circ \phi^{\circ k-i} \circ \alpha_{1}^{-1}$ is defined in a straight horizontal strip

$$
Y=\left\{z \in \mathbb{C}_{(-2 \rho, 1+2 \rho)}:|\operatorname{Im} z|<M\right\}
$$

for some $M>0$ independent of $n$. Since the space of normalized univalent maps of $Y$ is compact, the lemma is true if $\operatorname{dist}\left(z, J_{-i}\right) /\left|J_{-i}\right| \leq \rho$.

Now assume $\operatorname{dist}\left(z, J_{-i}\right) /\left|J_{-i}\right|>\rho$. Consider the smallest closed hyperbolic neighborhood $\overline{G_{\theta}\left(J_{-i}\right)}$ containing $z_{-i}$. For a point $\zeta \in \mathbb{C}_{I}$ with $\operatorname{dist}(\zeta, I)>\rho|I|$ and $\widehat{(\zeta, I)}>\epsilon$, the smallest closed neighborhood $\overline{D_{\gamma}(I)}$ containing $\zeta$ satisfies $\operatorname{diam} D_{\gamma}(I) \leq C(\rho, \epsilon) \operatorname{dist}(\zeta, I)$ (see Y Ya], Lemma 2.1). As $z_{-i} \in \breve{S}$ and $\left(\overline{z_{-i}, J_{-i}}\right)>$ $\epsilon$, compactness considerations imply that $\operatorname{diam} G_{\theta}\left(J_{-i}\right)<C(\rho, \epsilon, \breve{S}) \operatorname{dist}\left(z_{-i}, J_{-i}\right)$ and diam $D_{\theta}\left(J_{-i}\right)<C(\epsilon, \breve{S}) \operatorname{diam} G_{\theta}\left(J_{-i}\right)$. By the Schwarz Lemma, $z_{-k} \in \overline{G_{\theta}\left(J_{-k}\right)}$ $\subset \overline{D_{\theta}\left(J_{-k}\right)}$ and the claim follows.

For the rest of this section we adopt the following notations:

$$
\begin{aligned}
I_{m} & =\Upsilon\left(\left[1, B^{\circ q_{m}}(1)\right]\right), \\
T_{m} & =\Upsilon\left(\left[1, B^{\circ q_{m}-q_{m+1}}(1)\right]\right), \\
G_{m} & =G_{m, \alpha}=G_{\alpha}\left(\Upsilon\left(\left[B^{\circ q_{m+1}}(1), B^{q_{m}-q_{m+1}}(1)\right]\right)\right),
\end{aligned}
$$

where in the definition of the hyperbolic neighborhood $G_{m}$ we fix an angle $0<$ $\alpha<\pi / 2$ which will be specified later (just before Lemma 6.5 below). Note that $I_{m} \subset T_{m} \subset \Upsilon\left(\left[B^{\circ q_{m+1}}(1), B^{q_{m}-q_{m+1}}(1)\right]\right)$ and, by real a priori bounds, the three intervals have commensurable lengths.

Let us summarize some facts about the moments of closest returns which will be utilized in the following few lemmas.

Proposition 6.2. Consider the backward orbit (6.4) for a fixed $n$ and let $m \leq n-2$. Then, the collection

$$
J_{-q_{m+1}}, J_{-2 q_{m+1}}, \ldots, J_{-a_{m+2} q_{m+1}}, J_{-\left(a_{m+2}+1\right) q_{m+1}}
$$

represents the consecutive returns of the orbit (6.4) to $T_{m}$ before the second return to $T_{m+1}$, which is the interval $J_{-2 q_{m+2}}$. The first return to $T_{m+1}$ occurs in between the last two elements of (6.7). Moreover, if $\theta$ is of bounded type, so that $\sup _{\ell} a_{\ell}<\infty$, then all the intervals in (6.7) have lengths $K$-commensurable with $J_{0}$, with $K>1$ independent of $m$ and $n$.

Proof. The statements on the order of the closest returns follow from elementary combinatorial considerations. Let us address the issue of commensurability. By Świastek-Herman real a priori bounds, each of the intervals in (6.7) is well inside of the interval $T_{m}$. Hence, for $1 \leq k \leq a_{m+2}+1$ the derivative of the diffeomorphism $J_{-k q_{m+1}} \mapsto J_{-(k-1) q_{m+1}}$ is bounded by a positive constant independent of $k, m$ and $n$. Since $\sup _{\ell} a_{\ell}<\infty$, there exists $K>0$ independent of $m, n$ such that the intervals (6.7) are $K$-commensurable with $J_{0}$.

The following two lemmas are direct adaptations of Lemmas 4.2 and 4.4 of $[\mathrm{Ya}$. In both lemmas we work with the orbit (6.5) for some fixed value of $n$. 
Lemma 6.3. There exist positive constants $\epsilon_{1}$ and $C_{1}$ depending only on the choice of the angle $\alpha$ in the definition of $G_{m}$ such that the following holds: For $m \leq n-1$, let $J$ and $J^{\prime}$ be two consecutive returns of the orbit (6.4) to $T_{m}$ before the second return to $T_{m+1}$, and let $\zeta, \zeta^{\prime}$ be the corresponding points of the backward orbit (6.5). If $\zeta \in G_{m}$, then either $\zeta^{\prime} \in G_{m}$ or else $\left.\widehat{\left(\zeta^{\prime}, J^{\prime}\right.}\right)>\epsilon_{1}$ and $\operatorname{dist}\left(\zeta^{\prime}, J^{\prime}\right)<C_{1}\left|I_{m}\right|$.

We remark that in general the constants $\epsilon_{1}$ and $C_{1}$ will depend on the Blaschke product $B$.

Proof. Note that by Proposition [6.2, $J=J_{-i q_{m+1}}$ and $J^{\prime}=J_{-(i+1) q_{m+1}}$ for some $1 \leq i \leq a_{m+2}$. Let $\check{G}_{m}$ denote the pull-back of $G_{m}$ along the backward orbit $J, \ldots, J^{\prime}=\phi^{\circ q_{m+1}}(J)$. Also let $G_{m}^{\prime}$ denote the pull-back of $G_{m}$ along the orbit segment $J, \ldots, \phi^{\circ q_{m}-1}(J)$, and let $G_{m}^{\prime \prime}=\phi\left(G_{m}^{\prime}\right)$.

The combinatorics of closest returns implies that the restriction of $B^{-\left(q_{m}-1\right)}$ to $\left(B^{\circ q_{m+1}}(1), B^{q_{m}-q_{m+1}}(1)\right)$ is a diffeomorphism. Hence the pull-back of $G_{m}$ along the orbit $J, \ldots, \phi^{\circ q_{m}-1}(J)$ is univalent. By the Schwarz Lemma,

$$
G_{m}^{\prime} \subset G_{\alpha}\left(\Upsilon\left(\left[B^{\circ q_{m+1}-q_{m}+1}(1), B^{1-q_{m+1}}(1)\right]\right)\right) .
$$

By Świastek-Herman real a priori bounds, the critical value $\tau$ divides the interval $\Upsilon\left(\left[B^{\circ q_{m+1}-q_{m}+1}(1), B^{1-q_{m+1}}(1)\right]\right)$ into $K_{1}$-commensurable pieces, where $K_{1}$ becomes universal for large $m$, and can therefore be chosen independent of $m$. As the derivative of the exponential map is bounded away from 0 and $\infty$ on the strip $S$, the estimate (6.1) is still valid for the lifted map near the critical point. Together with elementary properties of the cube root map this implies that $G_{m}^{\prime \prime} \subset G_{\beta}\left(\Upsilon\left(\left[B^{\circ q_{m+1}-q_{m}}(1), 1\right]\right)\right)$ for some $\beta>0$ independent of $m$. Let $V_{0} \subset S$ be the union of the connected components of $\frac{ \pm 1}{2 \pi i} \log \left(\bar{U}_{1}\right)$ attached to 0 (see Figure 12). Since the boundary of $G_{m}^{\prime \prime}$ contains a segment of $\partial V_{0}$ which forms an angle $\pi / 3$ with $\mathbb{R}$ at 0 , we have $G_{m}^{\prime \prime} \subset G_{\gamma}\left(\Upsilon\left(\left[B^{\circ q_{m+1}-q_{m}}(1), a_{1}\right]\right)\right) \cup G_{\sigma}\left(\Upsilon\left(\left[a_{2}, 1\right]\right)\right)$, where the points $B^{\circ q_{m+1}-q_{m}}(1), a_{1}, a_{2}, 1$ form a $K_{2}$-bounded configuration with $K_{2}, \gamma>0$ and $\sigma>\pi / 2>\alpha$ independent of $m$.

The pull-back of $G_{m}^{\prime \prime}$ to $\check{G}_{m}$ is univalent. Applying the Schwarz Lemma, we have $\check{G}_{m} \subset G_{m} \cup G_{\gamma}\left(\Upsilon\left(\left[1, B^{-q_{m+1}+q_{m}}\left(a_{1}\right)\right]\right)\right)$ and the claim follows.

Lemma 6.4. There exist positive constants $\epsilon_{2}$ and $C_{2}$ depending only on the choice of $\alpha$ in the definition of $G_{m}$ such that the following holds: For $m \leq n-2$, let $J$ be the last return of the orbit (6.4) to the interval $T_{m}$ preceding the first return to $T_{m+1}$, let $J^{\prime}$ be the next return to $T_{m}$, and let $J^{\prime \prime}$ be the second return to $T_{m+1}$. Let $\zeta, \zeta^{\prime}$ and $\zeta^{\prime \prime}$ be the corresponding points in the backward orbit (6.5). If $\zeta^{\prime} \in G_{m}$, then either $\zeta^{\prime \prime} \in G_{m+1}$ or else $\left(\widehat{\zeta^{\prime \prime}, I_{m+1}}\right)>\epsilon_{2}$ and $\operatorname{dist}\left(\zeta^{\prime \prime}, J^{\prime \prime}\right)<C_{2}\left|I_{m+1}\right|$.

Proof. Note that by Proposition 6.2, the first return of the orbit (6.4) to $T_{m+1}$ is $J_{-q_{m+2}}$, and that $J^{\prime \prime}=J_{-2 q_{m+2}}, J^{\prime}=J_{-\left(a_{m+2}+1\right) q_{m+1}}=J_{-q_{m+2}-q_{m+1}+q_{m}}$ and $J=J_{-a_{m+2} q_{m+1}}=J_{-q_{m+2}+q_{m}}$. Let $\hat{J}=J_{-q_{m+2}-q_{m+1}}=\phi^{\circ q_{m}}\left(J^{\prime}\right)$ and $\hat{\zeta}$ be the corresponding element of (6.5) $)$. It is easily seen that $J^{\prime} \subset \Upsilon\left(\left[B^{\circ q_{m}}(1), B^{-q_{m+1}+q_{m}}(1)\right]\right)$, and $\hat{J} \subset\left[0, \Upsilon\left(B^{-q_{m+1}}(1)\right)\right]$. By the Schwarz Lemma and elementary properties of the map $B$ (see (6.1) ) , there exist points $b_{1}, b_{2}$ in $\left[1, B^{-q_{m+1}}(1)\right]$ such that $1, b_{1}, b_{2}$, and $B^{-q_{m+1}}(1)$ form a $K$-bounded configuration, and

$$
\hat{\zeta} \in G_{\sigma}\left(\Upsilon\left(\left[1, b_{1}\right]\right)\right) \cup G_{\gamma}\left(\Upsilon\left(\left[b_{2}, B^{-q_{m+1}}(1)\right]\right)\right)
$$




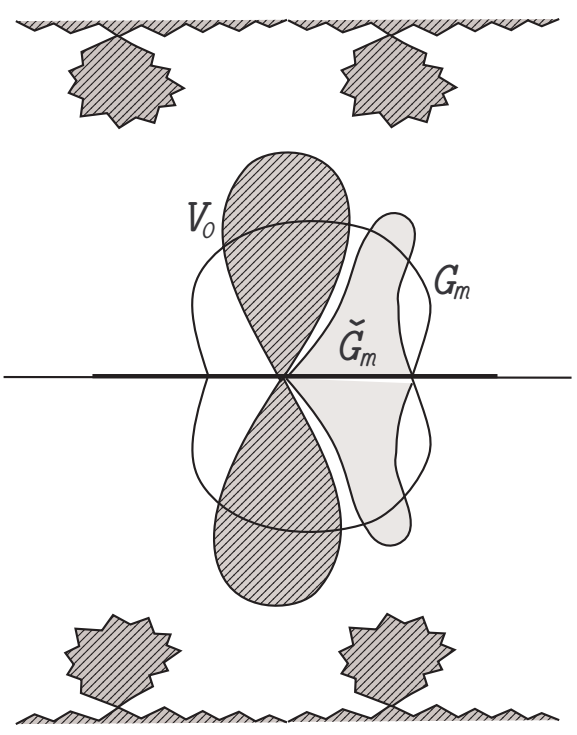

FiguRE 12.

for $\sigma>\pi / 2$ and $\gamma>0$ independent of $m$. Since the iterate $\phi^{\circ q_{m+2}-q_{m+1}}$ extends univalently to $S_{\Upsilon\left(\left(1, B^{\left.\left.-q_{m+1}(1)\right)\right)}\right.\right.}$, the claim follows from the Schwarz Lemma.

Before we proceed to the next lemma, let us make the following selections:

- The integer $N$. By Lemma [5.9, we may choose some $N \geq 1$ such that $P_{n} \cap$ $\partial U^{\infty}=\emptyset$ for all $n \geq N$.

- The strip $\breve{S}$ used in Lemma 6.1 Let $E$ be an annulus around the unit circle, compactly contained in the domain $\mathbb{C} \backslash\left(\overline{U^{0}} \cup \overline{U^{\infty}}\right)$, such that $P_{N} \cup P_{N+1} \subset E$. Then we choose $\breve{S}$ as the strip $\frac{1}{2 \pi i} \log (E)$. Note that $\breve{S} / \mathbb{Z} \Subset S / \mathbb{Z}$.

- The lifted puzzle-pieces $\hat{P}_{n}$. We denote by $\hat{P}_{n}$ the component of $\frac{1}{2 \pi i} \log \left(P_{n}\right)$ attached to $\Upsilon\left(\left[1, B^{-q_{n}}(1)\right]\right)$.

- The angle $\alpha$. We choose $0<\alpha<\pi / 2$ so that

$$
\hat{P}_{N+2} \cup \hat{P}_{N+3} \subset G_{\alpha}\left(\Upsilon\left(\left[B^{\circ q_{N+2}}(1), B^{q_{N+1}-q_{N+2}}(1)\right]\right)\right)=G_{N+1, \alpha}
$$

and we set $G_{n}=G_{n, \alpha}$ as in 6 .6.6.

- The constant $\epsilon^{*}$. We choose $\epsilon^{*}=\epsilon^{*}(\alpha)>0$ to be the smaller of the two constants $\epsilon_{1}, \epsilon_{2}$ from Lemmas 6.3 and 6.4 .

- The constant $C^{*}$. We choose $C^{*}=C^{*}(\alpha)>0$ to be the larger of the two constants $C_{1}, C_{2}$ from Lemmas 6.3 and 6.4

Note that by Corollary $5.5 P_{n+2} \subsetneq P_{n}$ for all $n$, hence $\hat{P}_{n} \subset G_{N+1}$ for all $n \geq N+2$.

Our argument culminates in the following estimate.

Lemma 6.5. Let $P_{n}$ denote the $n$-th critical puzzle-piece and let $N$ be as above. Then, there exist constants $K_{1}, K_{2}>1$ such that for every $n \geq N+3$ and every 
$z \in \hat{P}_{n-1}$ with the corresponding backward orbit $\left\{z_{-i}\right\}$ as in (6.5),

$$
\frac{\operatorname{dist}\left(z_{-\left(q_{n}-1\right)}, J_{-\left(q_{n}-1\right)}\right)}{\left|J_{-\left(q_{n}-1\right)}\right|} \leq K_{1} \frac{\operatorname{dist}\left(z, J_{0}\right)}{\left|J_{0}\right|}+K_{2} .
$$

As a result, there exist positive constants $A_{1}, A_{2}$ such that for all $n \geq N+3$,

$$
\frac{\operatorname{diam} P_{n}}{\left|\left[1, B^{-q_{n}}(1)\right]\right|} \leq A_{1} \sqrt[3]{\frac{\operatorname{diam} P_{n-1}}{\left|\left[B^{-q_{n-1}}(1), 1\right]\right|}}+A_{2} .
$$

Proof. Let $n \geq N+3$ and $z \in \hat{P}_{n-1}$. By the way $\alpha$ is chosen, there exists the largest integer $m$, with $N+1 \leq m \leq n$, such that $z \in G_{m}$. If $m=n$ or $m=n-1$ so that $z \in G_{n} \cup G_{n-1}$, then by the Schwarz Lemma the left side of (6.8) will be bounded by a universal constant and (6.8) will hold trivially. Hence, we may assume without loss of generality that $m \leq n-2$.

By compactness, for all $\ell$ the hyperbolic neighborhood $G_{\ell}$ is commensurable with $I_{\ell}$ which in turn is commensurable with $I_{\ell+1}$ by real a priori bounds. Since $z \in G_{m} \backslash G_{m+2}$, we see that $\operatorname{dist}\left(z, J_{0}\right)$ is commensurable with $\left|I_{m}\right|$.

By Proposition 6.2 for every $m \leq \ell \leq n-2$, the intervals

$$
J_{-q_{\ell+1}}, \ldots, J_{-\left(a_{\ell+2}+1\right) q_{\ell+1}}
$$

are the consecutive returns of the backward orbit (6.4) to $T_{\ell}$ before the second return to $T_{\ell+1}$ and are all $K$-commensurable with $J_{0}$, for a $K$ independent of $\ell$ and $n$.

We claim that either

(i) there exists a moment $i$ of the form $i=j q_{\ell+1}$ for some $1 \leq j \leq a_{\ell+2}+1$ and $m \leq \ell \leq n-2$ such that $\left(\widehat{z_{-i}, J_{-i}}\right)>\epsilon^{*}$ and $\operatorname{dist}\left(z_{-i}, J_{-i}\right)<C^{*}\left|I_{\ell}\right|$, or

(ii) $z_{-q_{n}} \in G_{\chi}\left(\left[\Upsilon\left(B^{\circ\left(q_{n-1}-q_{n-2}\right)}(1)\right), 0\right]\right)$ for some $\chi$ independent of $n$.

Suppose that (i) never occurs. Then we prove (ii) by an inductive argument. Set $k_{m}=0$, and for $m<\ell \leq n-3$ let $k_{\ell}=2 q_{\ell+1}$ so that $J_{-k_{\ell}}$ is the second return of the inverse orbit (6.4) to $T_{\ell}$. We have $z_{-k_{m}} \in G_{m}$ by the choice of $m$. Suppose $z_{-k_{\ell}} \in$ $G_{\ell}$, and let us show that $z_{-k_{\ell+1}} \in G_{\ell+1}$. Indeed, by Lemma 6.3 and our assumption, for all $k$ such that $k_{\ell} \leq k q_{\ell+1}<k_{\ell+1}$, we have $z_{-k q_{\ell+1}} \in G_{\ell}$ and the desired result follows from Lemma 6.4. By induction on $\ell$, we have $z_{-k_{n-3}}=z_{-2 q_{n-2}} \in G_{n-3}$. Now if $q_{n} \geq 2 q_{n-1}$, then one more step of the induction shows that $z_{-2 q_{n-1}} \in G_{n-2}$. By Lemma 6.3 $z_{-a_{n} q_{n-1}} \in G_{n-2}$ and by the Schwarz Lemma and elementary properties of the cube root map, we have $z_{-q_{n}} \in G_{\chi_{1}}\left(\left[\Upsilon\left(B^{\circ\left(q_{n-1}-q_{n-2}\right)}(1)\right), 0\right]\right)$ for some constant $\chi_{1}$ independent of $n$. In the case when $q_{n}=q_{n-1}+q_{n-2}$, Lemma 6.3 implies that $z_{-\left(a_{n-1}+1\right) q_{n-2}} \in G_{n-3}$. Again using the Schwarz Lemma and elementary properties of the cube root, we have

$$
z_{-q_{n}}=z_{-\left(a_{n-1}+1\right) q_{n-2}-q_{n-3}} \in G_{\chi_{2}}\left(\left[\Upsilon\left(B^{-q_{n-2}}(1)\right), 0\right]\right)
$$

for a constant $\chi_{2}$ independent of $n$. It follows that (ii) holds with $\chi=\min \left(\chi_{1}, \chi_{2}\right)$, and the proof of the claim is completed.

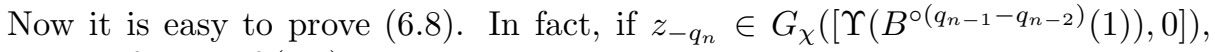
then the left side of (6.8) will be bounded by a universal constant, as can be easily seen from elementary properties of the cube root map. Otherwise, for some moment $i$ as in (i) above, we have $\left(\widehat{z_{-i}, J_{-i}}\right)>\epsilon^{*}$ and $\operatorname{dist}\left(z_{-i}, J_{-i}\right)<C^{*}\left|I_{\ell}\right|$ in which case 
it follows from Lemma 6.1 that

$$
\begin{aligned}
\frac{\operatorname{dist}\left(z_{-\left(q_{n}-1\right)}, J_{-\left(q_{n}-1\right)}\right)}{\left|J_{-\left(q_{n}-1\right)}\right|} & \leq C\left(\epsilon^{*}, \breve{S}\right) \frac{\operatorname{dist}\left(z_{-i}, J_{-i}\right)}{\left|J_{-i}\right|}<C\left(\epsilon^{*}, \breve{S}\right) C^{*} K \frac{\left|I_{\ell}\right|}{\left|J_{0}\right|} \\
& \leq K^{\prime} \frac{\left|I_{m}\right|}{\left|J_{0}\right|} \leq K^{\prime \prime} \frac{\operatorname{dist}\left(z, J_{0}\right)}{\left|J_{0}\right|} .
\end{aligned}
$$

It remains to derive the cubic estimate (6.9). Let

$$
\Pi_{0}=\hat{P}_{n-1}, \Pi_{-1}, \ldots, \Pi_{-q_{n}}=\hat{P}_{n}
$$

be the backward orbit of $\hat{P}_{n}$ corresponding to the orbit (6.4). From (6.8),

$$
\frac{\operatorname{diam} \Pi_{-\left(q_{n}-1\right)}}{\left|J_{-\left(q_{n}-1\right)}\right|} \leq 2 K_{1} \frac{\operatorname{diam} \hat{P}_{n-1}}{\left|J_{0}\right|}+2 K_{2}+1 .
$$

Together with the estimate [6.1), this implies 6.9].

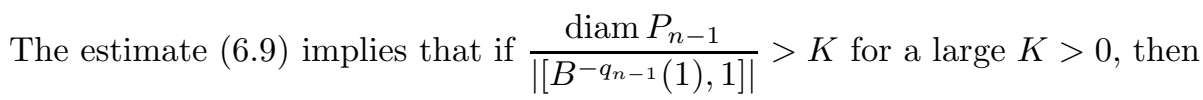

$$
1 \leq \frac{\operatorname{diam} P_{n}}{\left|\left[B^{-q_{n}}(1), 1\right]\right|} \leq \frac{1}{2} \cdot \frac{\operatorname{diam} P_{n-1}}{\left|\left[B^{-q_{n-1}}(1), 1\right]\right|} .
$$

It follows that for large $n$ the puzzle-piece $P_{n}$ is commensurable with its base arc $\left[B^{-q_{n}}(1), 1\right]$. By the claim in the proof of the previous lemma, combined with the Schwarz Lemma, there exists a constant $\sigma>0$ independent of $n$ such that $\hat{P}_{n} \subset G_{\sigma}\left(\left[\Upsilon\left(B^{\circ\left(q_{n-1}-q_{n-2}\right)}(1)\right), 0\right]\right)$. By the combinatorics of the closest returns the number of times the pull-back of $G_{\sigma}\left(\left[0, \Upsilon\left(B^{\circ\left(q_{n-2}-q_{n-3}\right)}(1)\right)\right]\right) \supset \hat{P}_{n-1}$ along the backward orbit (6.11) hits 0 is bounded by a constant independent of $n$. By the Schwarz Lemma and the elementary properties of the cube root map we have

Corollary 6.6. There exists an angle $0<\gamma<\pi / 2$ such that for all $n$,

$$
\hat{P}_{n} \subset G_{\gamma}\left(\Upsilon\left(\left[B^{-q_{n}}(1), 1\right]\right)\right) .
$$

Let us summarize the consequences. We first prove the following:

Lemma 6.7 (Only two drop-chains). There are exactly two drop-chains of the form $\mathcal{D}_{1}=\overline{\bigcup_{k} U_{\iota_{1} \ldots \iota_{k}}^{\infty}}$ and $\mathcal{D}_{2}=\overline{\bigcup_{k} U_{\iota_{1}^{\prime} \ldots \iota_{k}^{\prime}}^{\infty}}$ accumulating at the critical point 1 . Moreover, both of these drop-chains land at 1 , and they separate $U_{1}$ from $\mathbb{D}$, in the sense that $U_{1}$ and $\mathbb{D}$ belong to different components of $\widehat{\mathbb{C}} \backslash\left(\mathcal{D}_{1} \cup \mathcal{D}_{2}\right)$.

Proof. Let $\mathcal{D}=\overline{\bigcup_{k} U_{\iota_{1} \ldots \iota_{k}}^{\infty}}$ be any drop-chain accumulating at 1 . Then, for any given $n$ there is a drop $U_{\iota_{1} \ldots \iota_{k}}^{\infty} \subset \mathcal{D}$ which intersects the critical puzzle-piece $P_{n}$. Since $U_{\iota_{1} \ldots \iota_{k}}^{\infty}$ cannot intersect $\partial P_{n}, U_{\iota_{1} \ldots \iota_{k}}^{\infty} \subset P_{n}$. By Lemma 5.7, the whole limb $L_{\iota_{1} \ldots \iota_{k}}^{\infty}$ is contained in $P_{n}$. By Corollary 6.6, diam $P_{n} \rightarrow 0$, hence the drop-chain $\mathcal{D}$ lands at 1.

By Corollary 4.6 every puzzle-piece $P_{n}$ contains a drop growing from infinity $U_{\iota_{1} \ldots \iota_{k}}^{\infty}$. Since $P_{n+2} \subset P_{n}$ (Corollary [5.5) ) and $\stackrel{\circ}{P}_{n} \cap \stackrel{\circ}{P}_{n+1}=\emptyset$, there exist at least two distinct drop-chains landing at 1 (passing through the $P_{n}$ with even and odd $n$, respectively). Clearly these drop-chains separate $U_{1}$ from $\mathbb{D}$.

Assume that there is a third drop-chain landing at 1 . Then, there are two distinct drop-chains landing at the critical value $B(1)$. Evidently, the complement of the 
union of these drop-chains has a component $O$ which does not contain any of the drops $U_{i}$. This implies that $O \subset \cup B^{-n}\left(U^{\infty}\right)$, which is a contradiction.

The above lemma implies that for every $i \geq 1$ there are exactly two drop-chains $\mathcal{D}_{1}^{i}, \mathcal{D}_{2}^{i}$ accumulating at the point $x_{i}=B^{-i+1}(1) \in \mathbb{T}$. These drop-chains land at $x_{i}$ and separate $U_{i}$ from $\mathbb{D}$. We may now define, as in subsection 3.3 the wake with root $x_{i}$ to be the connected component $W_{i}$ of $\widehat{\mathbb{C}} \backslash\left(\mathcal{D}_{1}^{i} \cup \mathcal{D}_{2}^{i}\right)$ containing $U_{i}$. For the corresponding limb we clearly have $L_{i} \subset \bar{W}_{i}$. Due to the symmetry of the surgery (Corollary 4.5), all the objects we have defined have their symmetric counterparts. That is, there is a sequence of critical puzzle-pieces $P_{n}^{\infty}$ converging to the critical point $c \in \partial U^{\infty}$, wakes $W_{i}^{\infty} \supset U_{i}^{\infty}$ with $L_{i}^{\infty} \subset \bar{W}_{i}^{\infty}$, etc.

We now proceed to give the proof of Theorem [5.1, which will occupy the rest of the section. The first step is to prove it for limbs of a fixed generation:

Lemma 6.8. As before, let $L_{i}$ be the limb of generation 1 with root $x_{i} \in \mathbb{T}$. Then $\operatorname{diam} L_{i} \rightarrow 0$ as $i \rightarrow \infty$. As a result, for any fixed address $\iota_{1} \ldots \iota_{k}$, $\operatorname{diam} L_{\iota_{1} \ldots \iota_{k} i} \rightarrow 0$ as $i \rightarrow \infty$. A similar statement holds for limbs growing from infinity.

Proof. As before, let $P_{n}$ be the $n$-th critical puzzle-piece. By considering the dynamical partition of level $n$ for the homeomorphism $\left(\left.B\right|_{\mathbb{T}}\right)^{-1}$, we see that the union

$$
\bigcup_{j=0}^{q_{n}-1} B^{\circ q_{n}-j}\left(P_{n}\right) \cup \bigcup_{j=0}^{q_{n-1}-1} B^{\circ q_{n+1}-j}\left(P_{n+1}\right)
$$

covers the circle. By Corollary 6.6 and the Schwarz Lemma, each piece in the above union has diameter commensurable to its base arc, which uniformly tends to 0 as $n \rightarrow \infty$ by real a priori bounds. By considerations similar to Lemma 5.7 every limb $L_{i}$ with $i \geq q_{n-1}+q_{n}$ is contained in the above union. This proves diam $L_{i} \rightarrow 0$ as $i \rightarrow \infty$. The statement about diam $L_{\iota_{1} \ldots \iota_{k} i}$ is an immediate corollary of this case. The symmetry of the surgery (Corollary 4.5) implies the similar statements for limbs growing from infinity.

Next, we consider the case of nested limbs:

Lemma 6.9. Every nested sequence

$$
L_{\iota_{1}} \supset L_{\iota_{1} \iota_{2}} \supset \cdots \supset L_{\iota_{1} \ldots \iota_{k}} \supset \cdots
$$

has diameter tending to 0 . A similar statement holds for nested sequences of limbs growing from infinity.

It will be more convenient for us to prove that if $L_{\iota_{1}}^{\infty} \supset \cdots \supset L_{\iota_{1} \ldots \iota_{k}}^{\infty} \supset \cdots$, then $\operatorname{diam} L_{\iota_{1} \ldots \iota_{k}}^{\infty} \rightarrow 0$ as $k \rightarrow \infty$. Let $z$ be a point in the intersection of this sequence and denote by $z_{i}$ the forward iterate $B^{\circ i}(z)$. There are two possibilities:

- Case 1. There exist $n$ and $N$ such that for $i \geq N, z_{i} \notin P_{n} \cup P_{n+1} \cup P_{n}^{\infty} \cup P_{n+1}^{\infty}$. Since the rotation numbers $\theta, \nu$ are irrational, this means $\left\{z_{i}\right\}$ does not accumulate on $\mathbb{T} \cup \partial U^{\infty}$. Select a convergent subsequence $z_{i_{m}} \rightarrow \zeta$. Evidently, $\zeta$ does not belong to the boundary of any drop or drop growing from infinity. We claim that for some $j \geq 1$, the wake $W_{j}^{\infty}$ growing from infinity contains $\zeta$. If not, select a sequence of wakes $W_{j_{k}}^{\infty}$ for which $\zeta$ is an accumulation point. By the definition of a wake, the set $L$ of all the accumulation points of the sequence $W_{j_{k}}^{\infty}$ is connected, and $L \cap \partial U^{\infty} \neq \emptyset$. Moreover, $L$ is disjoint from the boundary of every drop or drop growing from infinity, with the exception of $\mathbb{D}$ and $U^{\infty}$, and $L$ does not contain any 
preimages of the fixed point $\beta$ (since the latter belongs to the wake $W_{1}^{\infty}$ ). Hence, if $B^{\circ k}(L) \cap P_{n}^{\infty} \neq \emptyset$ for some $k$ and $n$, then $B^{\circ k}(L) \subset P_{n}^{\infty}$. Since $\nu$ is irrational, for every $n \geq 1$ there exists an $\ell_{n}$ such that $B^{\circ \ell_{n}}(L) \cap P_{n}^{\infty} \neq \emptyset$. As $\operatorname{diam} P_{n}^{\infty} \rightarrow 0$, this implies that $B^{\circ \ell_{n}}(\zeta) \rightarrow \partial U^{\infty}$. Hence, $z_{i}$ accumulates on $\partial U^{\infty}$, contradictory to our assumption. This proves the existence of the wake $W_{j}^{\infty}$.

For large $m$, let $\Omega_{m}$ be the univalent pull-back of this wake $W_{j}^{\infty}$ along the orbit $z=z_{0}, z_{1}, \ldots, z_{i_{m}}$. Since $W_{j}^{\infty} \cap J(B) \neq \emptyset$, a well-known Shrinking Lemma (see for example [Lyu, Prop. 1.10) implies that $\operatorname{diam} \Omega_{m} \rightarrow 0$ as $m \rightarrow \infty$. For a fixed $m, U_{\iota_{1} \ldots \iota_{k}}^{\infty} \subset \Omega_{m}$ for all large $k$. By an analogue of Lemma 5.7 we must have $L_{\iota_{1} \ldots \iota_{k}}^{\infty} \subset \Omega_{m}$ for all large $k$. This proves $\operatorname{diam} L_{\iota_{1} \ldots \iota_{k}}^{\infty} \rightarrow 0$ as $k \rightarrow \infty$.

- Case 2. To fix the ideas, let us assume that the critical point 1 is an accumulation point of the orbit $\left\{z_{i}\right\}$. Let $z_{i_{n}}$ be the first point in this orbit which belongs to the puzzle-piece $P_{n}$. Since the orbit of $z$ accumulates on $1, z_{-i_{n}}$ cannot belong to the boundary of any drop growing from infinity, nor can it be a preimage of the fixed point $\beta$. Hence, either $z_{-i_{n}} \in \stackrel{\circ}{P}_{n}$ or $z_{-i_{n}}$ belongs to the part of the boundary of $P_{n}$ which is made up of the boundary arcs of drops. In the latter case, we may further assume that $z_{-i_{n}}$ does not coincide with the root of any drop, for in that case the result would be immediate from Lemma 6.7

As before, let $b_{n}=\mathcal{T}\left(P_{n}\right)$ be the reflection of $P_{n}$ through $\mathbb{T}$, and denote by

$$
Y_{0}=P_{n} \cup b_{n} \leftarrow Y_{-1} \leftarrow \cdots \leftarrow Y_{-i_{n}}
$$

the univalent preimages of $P_{n} \cup b_{n}$ along the backward orbit $z_{i_{n}}, \ldots, z_{0}=z$. Denote by $X_{-i} \subset Y_{-i}$ the preimage of $P_{n}$ alone.

Lemma 6.10. There exists at most one moment $i$, with $1 \leq i \leq i_{n}$, such that an element $Y_{-i}$ in (6.12) hits the critical point 1. Moreover, the pull-back (6.12) decomposes into two maps with bounded distortion and, possibly, one iterate of $B^{-1}$ near the critical value.

Proof. Let us prove the first statement. Note that if $Y_{-i} \cap \mathbb{T}=\emptyset$ for some $i<$ $q_{n+1}$, then the backward orbit (6.12) never hits the critical point for $1 \leq i \leq i_{n}$. Otherwise $Y_{-q_{n+1}}$ must be one of the two univalent preimages of the piece $Y_{-q_{n+1}+1}$ containing the critical value $B(1)$. One verifies directly, using the combinatorics of closest returns and Lemma 5.4, that one of these two preimages is contained in $P_{n} \cup b_{n}$ and the other one coincides with $P_{n+1} \cup b_{n+1}$. Thus, by minimality of $i_{n}, Y_{-q_{n+1}}=P_{n+1} \cup b_{n+1}$. The next possible moment when (6.12) can hit 1 is $i=q_{n+1}+q_{n}$. However, $Y_{-q_{n+1}-q_{n}} \cap \mathbb{T}=\emptyset$, since otherwise we can verify that $X_{-q_{n+1}-q_{n}} \subset P_{n}$ which is not possible by minimality of $i_{n}$.

Now let $k \leq i_{n}$ be the last moment when $Y_{-k} \cap \mathbb{T} \neq \emptyset$. As seen from the above argument, in combination with Świątek-Herman real a priori bounds and Corollary 6.6. the pull-back $Y_{0} \leftarrow \cdots \leftarrow Y_{-k}$ decomposes into two maps with bounded distortion and, possibly, one branch of $B^{-1}$ near the critical value. The combinatorics of closest returns and real a priori bounds also imply that $\operatorname{dist}\left(Y_{-k}, B(1)\right)$ is greater than $K_{1}$ diam $Y_{-k}$ for some constant $K_{1}>0$. Hence the distance from $Y_{-k-1}$ to $\mathbb{T} \cup \partial U^{\infty}$ is greater than $K_{2} \operatorname{diam} Y_{-k-1}$ for $K_{2}>0$, and the rest of the pull-back $Y_{-k} \leftarrow \cdots \leftarrow Y_{-i_{n}}$ has bounded distortion by the Koebe distortion theorem. 
By Lemma 5.8 and Corollary 6.6, the union $P_{n} \cup b_{n}$ contains a Euclidean disk, centered at a point in $\mathbb{T}$, whose diameter is commensurable with diam $P_{n}$. Therefore, by Lemma 6.10, the domain $Y_{-i_{n}} \ni z$ contains a Euclidean disk centered at a point of $J(B)$ whose diameter is commensurable with diam $Y_{-i_{n}}$. This implies that $\operatorname{diam} X_{-i_{n}} \leq \operatorname{diam} Y_{-i_{n}} \rightarrow 0$.

It follows from our previous remarks that $z$ belongs either to $\stackrel{\circ}{X}_{-i_{n}}$ or to the part of $\partial X_{-i_{n}}$ which is made up of the boundary arcs of drops, and $z$ is not the root of any drop. It is not hard to see that in either case, for a fixed $n$ and large $k$, we must have $U_{\iota_{1} \ldots \iota_{k}}^{\infty} \subset X_{-i_{n}}$. By Lemma 5.7 $L_{\iota_{1} \ldots \iota_{k}}^{\infty} \subset X_{-i_{n}}$, which implies $\operatorname{diam} L_{\iota_{1} \ldots \iota_{k}}^{\infty} \rightarrow 0$ as $k \rightarrow \infty$. This completes the proof of Lemma 6.9.

Proof of Theorem 5.1. By symmetry of the surgery (Corollary 4.5), it suffices to prove the result for limbs growing from infinity, that is, to show

$$
\sup _{\iota_{1}+\cdots+\iota_{k}=d} \operatorname{diam} L_{\iota_{1}, \ldots, \iota_{k}}^{\infty} \rightarrow 0, \text { as } d \rightarrow \infty .
$$

Assume the contrary. Then there exists a sequence of limbs growing from infinity $L^{\infty}(n)=L_{\iota_{1, n} \ldots \iota_{k(n), n}}^{\infty}$ such that $\inf _{n} \operatorname{diam} L^{\infty}(n)>0$ and $\iota_{1, n}+\cdots+\iota_{k(n), n} \rightarrow \infty$ as $n \rightarrow \infty$. If $\left\{\iota_{1, n}\right\}$ is an unbounded sequence, we obtain an immediate contradiction with Lemma 6.8 Therefore, by passing to a subsequence, we may assume that $\iota_{1, n}=\iota_{1}$ is constant. Along this subsequence, if $\left\{\iota_{2, n}\right\}$ is unbounded, we obtain a contradiction with Lemma 6.8. Hence by passing to a further subsequence, we may assume that $\iota_{2, n}=\iota_{2}$ is constant. Continuing inductively, we obtain a sequence $\left\{\iota_{j}\right\}$ and a subsequence $L^{\infty}\left(n_{j}\right)$ such that $L^{\infty}\left(n_{j}\right) \subset L_{\iota_{1}, \ldots, \iota_{j}}^{\infty}$. However, the nested sequence $L_{\iota_{1}}^{\infty} \supset L_{\iota_{1}, \iota_{2}}^{\infty} \supset \cdots \supset L_{\iota_{1}, \ldots, \iota_{j}}^{\infty} \supset \cdots$ has shrinking diameter by Lemma 6.9 which contradicts inf $\operatorname{diam}^{\infty}\left(n_{j}\right)>0$. This completes the proof of Theorem 5.1

\section{The proof of the Main Theorem}

Throughout this section we fix a pair of irrationals $\theta$ and $\nu$ of bounded type, with $\theta \neq 1-\nu$. In what follows we prove the Main Theorem, that is, we show that the quadratic rational map $F_{\theta, \nu}$ of (2.1) is in fact the mating of the quadratic polynomials $f_{\theta}$ and $f_{\nu}$ in the sense we described in the introduction.

7.1. Spines and itineraries. Let $\tilde{Q}_{\theta}$ be the modified Blaschke product of (3.2). Consider the two drop-chains

$$
\mathcal{C}=\overline{U_{0} \cup U_{1} \cup U_{11} \cup \cdots}, \quad \mathcal{C}^{\prime}=\overline{U_{0} \cup U_{2} \cup U_{21} \cup \cdots}
$$

with $\tilde{Q}_{\theta}\left(\mathcal{C}^{\prime}\right)=\mathcal{C}$. Applying Lemma 5.2 again, we see that $\mathcal{C}$ and $\mathcal{C}^{\prime}$ land respectively at the repelling fixed point $\beta$ and its preimage $\beta^{\prime}$. By the spine of $\tilde{Q}_{\theta}$ we mean the union of the drop-rays

$$
S_{\theta}=R(\mathcal{C}) \cup R\left(\mathcal{C}^{\prime}\right)
$$

(compare Figure13, where the image of the spine of $\tilde{Q}_{\theta}$ is shown in the filled Julia set of the quadratic polynomial $f_{\theta}$ for $\left.\theta=(\sqrt{5}-1) / 2\right)$. Every point on the spine which is not in the interior of $K\left(\tilde{Q}_{\theta}\right)$ is either one of the endpoints $\beta$, $\beta^{\prime}$, or a preimage of the critical point $z=1$. 
By Petersen's Theorem 3.5 the Julia set $J\left(\tilde{Q}_{\theta}\right)$ is locally-connected. Thus the Böttcher map extends continuously from the basin of infinity of $\tilde{Q}_{\theta}$ to its boundary. As a consequence, there exists a Carathéodory loop $\eta_{\theta}: \mathbb{T} \rightarrow J\left(\tilde{Q}_{\theta}\right)$ which conjugates the angle-doubling map to $\tilde{Q}_{\theta}$. A point $z \in J\left(\tilde{Q}_{\theta}\right)$ is the landing point of an external ray $R^{e}(t)$ if and only if $\eta_{\theta}(t)=z$. It is easy to see that $\eta_{\theta}(0)=\beta$ and $\eta_{\theta}(1 / 2)=\beta^{\prime}$.

By Lemma 3.3 the critical point $z=1$, hence every preimage of it, is biaccessible, i.e., is the landing point of exactly two external rays. For the quadratic polynomial $f_{\theta}$ the converse statement is true for an arbitrary $\theta$ of Brjuno type: Every biaccessible point in the Julia set $J\left(f_{\theta}\right)$ eventually hits the critical point [Za1]. The two external rays landing at the critical point of $\tilde{Q}_{\theta}$ are both mapped to the external ray landing at the critical value $\tilde{Q}_{\theta}(1)$. This means that they have angles of the form $\omega / 2$ and $(\omega+1) / 2$, where $\omega=\omega(\theta)$ is a well-defined irrational number in the interval $(0,1)$. It can be shown that the function $\theta \mapsto \omega(\theta)$ is effectively computable (see [A] or [BS, and compare with subsection [8.2).

Consider the two connected subsets of the Julia set:

$$
\begin{aligned}
& J_{\theta}^{0}=\left\{z \in J\left(\tilde{Q}_{\theta}\right): z=\eta_{\theta}(t) \text { for some } 0 \leq t \leq 1 / 2\right\} \\
& J_{\theta}^{1}=\left\{z \in J\left(\tilde{Q}_{\theta}\right): z=\eta_{\theta}(t) \text { for some } 1 / 2 \leq t \leq 1\right\} .
\end{aligned}
$$

By local-connectivity of $J\left(\tilde{Q}_{\theta}\right)$ (Theorem 3.5), $J_{\theta}^{0} \cup J_{\theta}^{1}=J\left(\tilde{Q}_{\theta}\right)$, and evidently

$$
\begin{aligned}
J_{\theta}^{0} \cap J_{\theta}^{1} & =J\left(\tilde{Q}_{\theta}\right) \cap S_{\theta} \\
& =\left\{\beta, \beta^{\prime}\right\} \cup\left\{1=x_{1}, x_{11}, x_{111}, \ldots\right\} \cup\left\{x_{2}, x_{21}, x_{211}, \ldots\right\},
\end{aligned}
$$

which consists of the pair $\left\{\beta, \beta^{\prime}\right\}$ as well as all the biaccessible points along the spine. In particular, if a point $z \in J\left(\tilde{Q}_{\theta}\right)$ is neither a preimage of the fixed point $\beta$ nor biaccessible, then each point in the forward orbit of $z$ belongs either to $J_{\theta}^{0}$ or to $J_{\theta}^{1}$ (but not both).

We proceed to define the itinerary of a point $z \in J\left(\tilde{Q}_{\theta}\right)$ with respect to $S_{\theta}$. This will be a dynamically-defined infinite sequence of 0's and 1's which gives the binary expansion of the angle of an external ray landing at $z$. When $z$ is a preimage of $\beta$ or is biaccessible, we will assign two different itineraries to $z$ (see [Do1] for a general discussion on how one computes angles in similar situations). We would like to remark that it is much easier to define itineraries by constructing the standard dyadic partition for the map $w \mapsto w^{2}$ outside the unit disk and transfer it back to the basin of infinity for $\tilde{Q}_{\theta}$ using the Böttcher map. However, the following construction will be carried out internally, using only the Julia set, ignoring any information given by the basin of infinity. This point will be crucial in the proof of the main theorem.

Set $z_{0}=z, z_{i}=\tilde{Q}_{\theta}\left(z_{i-1}\right)$ for $i \geq 1$. We consider three distinct cases:

- Case 1. The orbit of $z$ never hits the spine $S_{\theta}$. In particular, $z$ is not biaccessible and hence there exists a unique angle $t$ with $z=\eta_{\theta}(t)$. Define the itinerary of $z$ to be the sequence $\varepsilon=\left(\varepsilon_{0}, \varepsilon_{1}, \varepsilon_{2}, \ldots\right)$, where $\varepsilon_{i} \in\{0,1\}$ is determined by the condition

$$
z_{i} \in J_{\theta}^{\varepsilon_{i}}, \quad i=0,1,2, \ldots
$$

Then it is easy to see that the angle $t$ has the binary expansion $0 . \varepsilon_{0} \varepsilon_{1} \varepsilon_{2} \ldots$ 
- Case 2. The orbit of $z$ eventually hits the fixed point $\beta$. Let us first consider the cases $z=\beta$ and $z=\beta^{\prime}$. The two itineraries of $\beta$ are given by

$$
\begin{aligned}
& \varepsilon(\beta)=(0,0,0,0, \ldots), \\
& \varepsilon^{\prime}(\beta)=(1,1,1,1, \ldots) .
\end{aligned}
$$

Similarly, for $\beta^{\prime}$ we set

$$
\begin{aligned}
& \varepsilon\left(\beta^{\prime}\right)=(0,1,1,1, \ldots), \\
& \varepsilon^{\prime}\left(\beta^{\prime}\right)=(1,0,0,0, \ldots) .
\end{aligned}
$$

Note that both itineraries in either case give the binary digits of the angle of the unique external ray landing at the corresponding point (on $\mathbb{R} / \mathbb{Z}$ and in base 2 , we have $0=0.0000 \ldots=0.1111 \ldots$ and $1 / 2=0.1000 \ldots=0.0111 \ldots)$.

More generally, suppose that $z_{n}=\beta$ and $n \geq 2$ is the smallest integer with this property. Then the two itineraries of $z$ will be of the form

$$
\begin{aligned}
\varepsilon & =\left(\varepsilon_{0}, \varepsilon_{1}, \ldots, \varepsilon_{n-2}, 0,1,1,1, \ldots\right), \\
\varepsilon^{\prime} & =\left(\varepsilon_{0}, \varepsilon_{1}, \ldots, \varepsilon_{n-2}, 1,0,0,0, \ldots\right),
\end{aligned}
$$

where the $\varepsilon_{i}$ are determined by the condition

$$
z_{i} \in J_{\theta}^{\varepsilon_{i}}, \quad i=0,1, \ldots, n-2 .
$$

Both itineraries of $z$ give the binary digits of the angle $t$ of the unique external ray landing at $z$ (we end up with two itineraries simply because such dyadic angles $t$ have two binary representations).

- Case 3. The orbit of $z$ eventually hits the critical point at 1 . In this case there are exactly two angles $0<t<s<1$ with $\eta_{\theta}(t)=\eta_{\theta}(s)=z$. Let us assume that the angles corresponding to the critical point have binary expansions $\omega / 2=0.0 \omega_{1} \omega_{2} \ldots$ and $(\omega+1) / 2=0.1 \omega_{1} \omega_{2} \ldots$ Then the critical value $v=\tilde{Q}_{\theta}(1)$ has a unique ray landing on it with angle $\omega=0 . \omega_{1} \omega_{2} \ldots$ Since the forward orbit of $v$ can never hit the spine, by Case 1 above, the binary digits of $\omega$ are uniquely determined by the condition

$$
\tilde{Q}_{\theta}^{\circ i}(1) \in J_{\theta}^{\omega_{i}}, \quad i=1,2,3, \ldots
$$

Let us first consider the case where $z$ itself belongs to the spine. If $z=x_{11 \cdots 1} \in$ $\tilde{Q}_{\theta}^{-k+1}(1)$ with $k \geq 1$, then the two itineraries of $z$ will be

$$
\begin{aligned}
& \varepsilon=(\underbrace{0,0, \ldots, 0}_{k \text { times }}, \omega_{1}, \omega_{2}, \ldots), \\
& \varepsilon^{\prime}=(\underbrace{1,1, \ldots, 1}_{k \text { times }}, \omega_{1}, \omega_{2}, \ldots) .
\end{aligned}
$$

Then $\varepsilon$ and $\varepsilon^{\prime}$ give the binary digits of $t$ and $s$, respectively. If, on the other hand, $z=x_{211 \cdots 1} \in \tilde{Q}_{\theta}^{-k}(1)$ with $k \geq 1$, then the two itineraries of $z$ will be

$$
\begin{aligned}
& \varepsilon=(0, \underbrace{1, \ldots, 1}_{k \text { times }}, \omega_{1}, \omega_{2}, \ldots), \\
& \varepsilon^{\prime}=(1, \underbrace{0, \ldots, 0}_{k \text { times }}, \omega_{1}, \omega_{2}, \ldots) .
\end{aligned}
$$


Again, $\varepsilon$ and $\varepsilon^{\prime}$ give the binary digits of $t$ and $s$, respectively. This finishes the definition of itineraries for preimages of 1 along the spine.

Finally, consider a point $z$ off the spine whose orbit eventually hits the critical point 1 . Let $n \geq 1$ be the smallest integer such that $z_{n} \in S_{\theta} \backslash\left\{\beta, \beta^{\prime}\right\}$. The orbit segment $z_{i}$ for $0 \leq i \leq n-1$ is off the spine so there is a well-defined $\varepsilon_{i} \in\{0,1\}$ with $z_{i} \in J_{\theta}^{\varepsilon_{i}}$. Follow this initial segment by the two itineraries of $z_{n}$ already defined above. Thus the two itineraries of $z$ are given by

$$
\begin{aligned}
& \varepsilon=(\varepsilon_{0}, \ldots, \varepsilon_{n-1}, \underbrace{\varepsilon_{n}, \varepsilon_{n+1}, \ldots}_{\varepsilon \text {-itinerary of } z_{n}}), \\
& \varepsilon^{\prime}=(\varepsilon_{0}, \ldots, \varepsilon_{n-1}, \underbrace{\varepsilon_{n}^{\prime}, \varepsilon_{n+1}^{\prime}, \ldots}_{\varepsilon^{\prime} \text {-itinerary of } z_{n}}) .
\end{aligned}
$$

The itineraries $\varepsilon$ and $\varepsilon^{\prime}$ then give the binary digits of the two angles $t$ and $s$, respectively.

Since $\tilde{Q}_{\theta}$ and $f_{\theta}$ are quasiconformally conjugate for $\theta$ of bounded type, with the conjugacy being conformal in the basin of infinity, we have a completely similar description for the spine and itineraries of points in the quadratic Julia set $J\left(f_{\theta}\right)$. Figure 13 (on the next page) shows the spine and selected rays for $f_{\theta}$ with $\theta=$ $(\sqrt{5}-1) / 2$.

We summarize the above discussion in the following proposition:

Proposition 7.1. (i) Let $z \in J\left(\tilde{Q}_{\theta}\right)$. Then the angle(s) of the external ray $(s)$ landing at $z$ is (are) determined by the itinerary(ies) of $z$, that is, by the answer to the purely topological question of whether points in the forward orbit of $z$ belong to $J_{\theta}^{0}, J_{\theta}^{1}$, or to which point of the spine. In particular, two points in the Julia set having the same itinerary must coincide.

(ii) Every infinite sequence of 0 's and 1's can be realized as the itinerary of a unique point in $J\left(\tilde{Q}_{\theta}\right)$.

7.2. Main reduction. A key ingredient in the proof of the main theorem is the following reduction step:

Theorem 7.2. Let $0<\theta, \nu<1$ be irrationals of bounded type and $\theta \neq 1-\nu$. Then there exist continuous maps $\zeta_{\theta}: K\left(\tilde{Q}_{\theta}\right) \rightarrow \widehat{\mathbb{C}}$ and $\zeta_{\nu}: K\left(\tilde{Q}_{\nu}\right) \rightarrow \widehat{\mathbb{C}}$ such that

$$
\begin{array}{lll}
\zeta_{\theta} \circ \tilde{Q}_{\theta} & =\tilde{B}_{\theta, \nu} \circ \zeta_{\theta} & \text { on } K\left(\tilde{Q}_{\theta}\right), \\
\zeta_{\nu} \circ \tilde{Q}_{\nu}=\tilde{B}_{\theta, \nu} \circ \zeta_{\nu} & \text { on } K\left(\tilde{Q}_{\nu}\right) .
\end{array}
$$

$\zeta_{\theta}$ and $\zeta_{\nu}$ can be chosen to be quasiconformal homeomorphisms in the interiors of $K\left(\tilde{Q}_{\theta}\right)$ and $K\left(\tilde{Q}_{\nu}\right)$, respectively. Moreover, $\zeta_{\theta}\left(K\left(\tilde{Q}_{\theta}\right)\right) \cup \zeta_{\nu}\left(K\left(\tilde{Q}_{\nu}\right)\right)=\widehat{\mathbb{C}}$ and $\zeta_{\theta}(z)=\zeta_{\nu}(w)$ if and only if there exists an angle $t \in \mathbb{T}$ such that $z=\eta_{\theta}(t)$ and $w=\eta_{\nu}(-t)$.

Before starting the proof, we fix some notation. For simplicity, we set $K\left(\tilde{Q}_{\theta}\right)=$ $K_{\theta}, K\left(\tilde{Q}_{\nu}\right)=K_{\nu}$. We also recall the definition of the compact set $K\left(\tilde{B}_{\theta, \nu}\right)=K_{\theta, \nu}$ as the set of all points whose forward orbits under the iteration of $\tilde{B}_{\theta, \nu}$ never hit the Siegel disk $U^{\infty}$. Similarly, $K_{\theta, \nu}^{\infty}=\overline{\mathbb{C} \backslash K_{\theta, \nu}}$ is the set of points whose forward orbits never hit the "Siegel disk" $U_{0}=\mathbb{D}$.

Proof of Theorem 7.2. We begin by constructing $\zeta_{\theta}$. The map $\zeta_{\nu}$ can be constructed in a similar fashion. Consider the modified Blaschke products $\tilde{Q}_{\theta}$ of (3.2) 


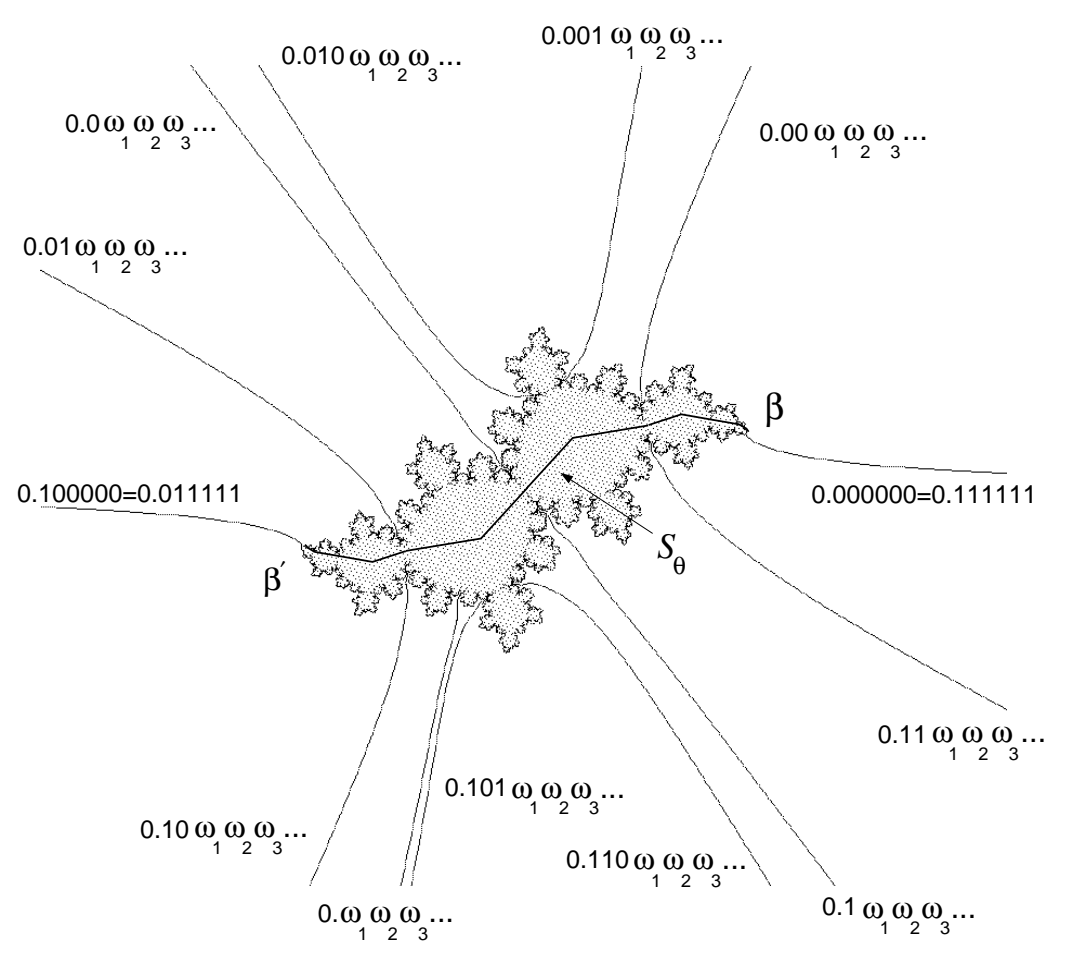

Figure 13. This picture shows the filled Julia set of the quadratic polynomial $f_{\theta}$ for $\theta=(\sqrt{5}-1) / 2$. The spine is shown by a thick path connecting the repelling fixed point $\beta$ to its preimage $\beta^{\prime}$. Selected rays and angles in base 2 are shown. Here $\omega=0 . \omega_{1} \omega_{2} \omega_{3} \ldots$ is the unique angle corresponding to the ray which lands at the critical value. For this value of $\theta, \omega$ is given by the continued fraction $\left[1,2,2,2^{2}, 2^{3}, 2^{5}, \ldots\right]$, where the powers of 2 form the Fibonacci sequence. Hence $\omega_{1}=1, \omega_{2}=0, \omega_{3}=1$, etc.

and $\tilde{B}_{\theta, \nu}$ of (4.9). Since both of these maps are quasiconformally conjugate to the rigid rotation $z \mapsto e^{2 \pi i \theta} z$ on the unit disk, one can define a quasiconformal conjugacy $\zeta_{\theta}: \mathbb{D} \rightarrow \mathbb{D}$ between them, which extends homeomorphically to a conjugacy $\zeta_{\theta}: \overline{\mathbb{D}} \rightarrow \overline{\mathbb{D}}$. This $\zeta_{\theta}$ can be extended to the union of the closures of all drops of $\tilde{Q}_{\theta}$ by pulling back. To this end, let $U_{\iota_{1} \ldots \iota_{k}}$ be any drop of $\tilde{Q}_{\theta}$ of generation $k$ and consider the corresponding drop $U_{\iota_{1} \ldots \iota_{k}}^{\prime}$ of $\tilde{B}_{\theta, \nu}$ with the same address. Let

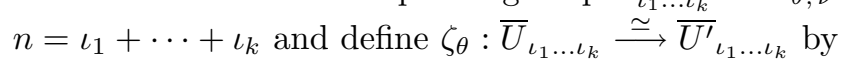

$$
\zeta_{\theta}=\tilde{B}_{\theta, \nu}^{-n} \circ \zeta_{\theta} \circ \tilde{Q}_{\theta}^{\circ n} .
$$

An easy induction on $n$ shows that $\zeta_{\theta}$ defined this way is a conjugacy between $\tilde{Q}_{\theta}$ and $\tilde{B}_{\theta, \nu}$ on $\bigcup_{k} \bigcup_{\iota_{1}, \ldots, \iota_{k}} \bar{U}_{\iota_{1} \ldots \iota_{k}}$ which is quasiconformal on the union

$$
\bigcup_{k} \bigcup_{\iota_{1}, \ldots, \iota_{k}} U_{\iota_{1} \ldots \iota_{k}}=\operatorname{int}\left(K_{\theta}\right)
$$

We would like to extend $\zeta_{\theta}$ to a continuous semiconjugacy $K_{\theta} \rightarrow K_{\theta, \nu}$. By Proposition 3.8, every point in $K_{\theta}$ is either in the closure of a drop or is the landing 
point of a unique drop-chain. Since $\zeta_{\theta}$ is already defined on $\bigcup_{k} \bigcup_{\iota_{1}, \ldots, \iota_{k}} \bar{U}_{\iota_{1} \ldots \iota_{k}}$, it suffices to define it at the landing points of drop-chains of $\tilde{Q}_{\theta}$. Take a dropchain $\mathcal{C}=\overline{\bigcup_{k} U_{\iota_{1} \ldots \iota_{k}}}$ which lands at $p$ and consider the corresponding drop-chain of $\tilde{B}_{\theta, \nu}, \mathcal{C}^{\prime}=\overline{\bigcup_{k} U_{\iota_{1} \ldots \iota_{k}}^{\prime}}$, whose drops have the same addresses. By Theorem 5.1, the diameters of the corresponding limbs $L_{\iota_{1} \ldots \iota_{k}}^{\prime}$ go to zero as $k \rightarrow \infty$, hence $\mathcal{C}^{\prime}$ lands at a well-defined point $p^{\prime} \in K_{\theta, \nu}$. Define $\zeta_{\theta}(p)=p^{\prime}$.

Evidently $\zeta_{\theta}$ defined this way has the property that for any $\operatorname{limb} L_{\iota_{1} \ldots \iota_{k}}$ of $\tilde{Q}_{\theta}$, the image $\zeta_{\theta}\left(L_{\iota_{1} \ldots \iota_{k}}\right)$ is precisely the limb $L_{\iota_{1} \ldots \iota_{k}}^{\prime}$ of $\tilde{B}_{\theta, \nu}$ with the same address. We would like to show that $\zeta_{\theta}$ is continuous as a map from $K_{\theta}$ into $\widehat{\mathbb{C}}$. Take a point $p \in K_{\theta}$ and a sequence $p_{n} \in K_{\theta}$ converging to $p$. When $p$ belongs to the interior of $K_{\theta}$ continuity is trivial. So let us assume that $p \in \partial K_{\theta}$. By Proposition 3.8, we have two possibilities:

- Case 1. $p$ is the landing point of a drop-chain $\mathcal{C}=\overline{\bigcup_{k} U_{\iota_{1} \ldots \iota_{k}}}$. Fix a multiindex $\iota_{1} \cdots \iota_{k}$ and observe that $p$ belongs to the wake $W_{\iota_{1} \ldots \iota_{k}}$. Therefore, for $n$ large enough, $p_{n}$ also belongs to $W_{\iota_{1} \ldots \iota_{k}}$. In particular, $p_{n} \in L_{\iota_{1} \ldots \iota_{k}}$, which implies $\zeta_{\theta}\left(p_{n}\right) \in L_{\iota_{1} \ldots \iota_{k}}^{\prime}$. It follows that $\operatorname{dist}\left(\zeta_{\theta}(p), \zeta_{\theta}\left(p_{n}\right)\right) \leq \operatorname{diam}\left(L_{\iota_{1} \ldots \iota_{k}}^{\prime}\right)$. Since $\operatorname{diam}\left(L_{\iota_{1} \ldots \iota_{k}}^{\prime}\right) \rightarrow 0$ as $k \rightarrow \infty$ by Theorem 5.1 we have $\zeta_{\theta}\left(p_{n}\right) \rightarrow \zeta_{\theta}(p)$ as $n \rightarrow \infty$.

- Case 2. $p$ belongs to the boundary of a drop $U_{\iota_{1} \ldots \iota_{k}}$ of $\tilde{Q}_{\theta}$ of smallest possible generation. It might be the case that $p$ is the root of a child $U_{\iota_{1} \ldots \iota_{k} \iota_{k+1}}$ in which case $\{p\}=\partial U_{\iota_{1} \ldots \iota_{k}} \cap \partial U_{\iota_{1} \ldots \iota_{k} \iota_{k+1}}$. If for all sufficiently large $n, p_{n}$ belongs to $\bar{U}_{\iota_{1} \ldots \iota_{k}}$ (or to $\bar{U}_{\iota_{1} \ldots \iota_{k}} \cup \bar{U}_{\iota_{1} \ldots \iota_{k} \iota_{k+1}}$ if $p$ is the root of $U_{\iota_{1} \ldots \iota_{k} \iota_{k+1}}$ ), then $\zeta_{\theta}\left(p_{n}\right) \rightarrow$ $\zeta_{\theta}(p)$ is immediate. Hence it suffices to prove the convergence in the case $p_{n} \notin$ $\bar{U}_{\iota_{1} \ldots \iota_{k}}$ (or $p_{n} \notin \bar{U}_{\iota_{1} \ldots \iota_{k}} \cup \bar{U}_{\iota_{1} \ldots \iota_{k} \iota_{k+1}}$ if $p$ is the root of $U_{\iota_{1} \ldots \iota_{k} \iota_{k+1}}$ ). Under this assumption, it follows from $p_{n} \rightarrow p$ that $p_{n}$ belongs to a limb $L(n)$ with root $x(n) \in \partial U_{\iota_{1} \ldots \iota_{k}}\left(\right.$ or $x(n) \in \partial U_{\iota_{1} \ldots \iota_{k}} \cup \partial U_{\iota_{1} \ldots \iota_{k} \iota_{k+1}}$ if $p$ is the root of $\left.U_{\iota_{1} \ldots \iota_{k} \iota_{k+1}}\right)$ such that $x(n) \rightarrow p$ as $n \rightarrow \infty$. Then $\zeta_{\theta}\left(p_{n}\right)$ belongs to the limb $L^{\prime}(n)$ of $\tilde{B}_{\theta, \nu}$ with the same address as $L(n)$ whose root $x^{\prime}(n)=\zeta_{\theta}(x(n))$ converges to $\zeta_{\theta}(p)$ as $n \rightarrow \infty$. Since $\operatorname{diam}\left(L^{\prime}(n)\right) \rightarrow 0$ by Theorem [5.1, we must have $\zeta_{\theta}\left(p_{n}\right) \rightarrow \zeta_{\theta}(p)$ as $n \rightarrow \infty$ as well. This finishes the proof of continuity.

We can define $\zeta_{\nu}$ and prove its continuity in a similar way. It is clear from the above construction that the semiconjugacy relations (7.2) hold and $\zeta_{\theta}\left(K_{\theta}\right)=K_{\theta, \nu}$ and similarly $\zeta_{\nu}\left(K_{\nu}\right)=K_{\theta, \nu}^{\infty}$.

It remains to prove the last property of $\zeta_{\theta}$ and $\zeta_{\nu}$. Consider the spines $S_{\theta}$ and $S_{\nu}$ for $\tilde{Q}_{\theta}$ and $\tilde{Q}_{\nu}$ as in subsection 7.1 and map them to get simple $\operatorname{arcs} \Sigma_{\theta}=\zeta_{\theta}\left(S_{\theta}\right)$ and $\Sigma_{\nu}=\zeta_{\nu}\left(S_{\nu}\right)$ (compare Figure 10). Set

$$
\Sigma=\Sigma_{\theta} \cup \Sigma_{\nu}
$$

Lemma 7.3. Two simple curves $\Sigma_{\theta}$ and $\Sigma_{\nu}$ only intersect at the two end-points $\beta$ and $\beta^{\prime}$. Hence $\Sigma$ is a Jordan curve on the Riemann sphere.

Proof. Any point in the intersection $\Sigma_{\theta} \cap \Sigma_{\nu}$ which is not $\beta$ or $\beta^{\prime}$ must be a $B_{\theta, \nu^{-}}$ preimage of both 1 and $c$, where $c$ is the critical point of $B_{\theta, \nu}$ on the boundary of $U^{\infty}$. But this is impossible since 1 and $c$ have disjoint forward orbits.

Now consider the four connected sets

$$
\Lambda_{\theta}^{i}=\zeta_{\theta}\left(J_{\theta}^{i}\right), \Lambda_{\nu}^{i}=\zeta_{\nu}\left(J_{\nu}^{i}\right), \quad i=0,1,
$$


where $J_{\theta}^{i}$ and $J_{\nu}^{i}$ are the subsets of the Julia sets $J\left(\tilde{Q}_{\theta}\right)$ and $J\left(\tilde{Q}_{\nu}\right)$ we defined in (17.1). Let

$$
X=\left\{\beta, \beta^{\prime}, 1=x_{1}, x_{11}, x_{111}, \ldots, x_{2}, x_{21}, x_{211}, \ldots\right\}
$$

and

$$
Y=\left\{\beta, \beta^{\prime}, c=x_{1}^{\infty}, x_{11}^{\infty}, x_{111}^{\infty}, \ldots, x_{2}^{\infty}, x_{21}^{\infty}, x_{211}^{\infty}, \ldots\right\} .
$$

It is clear from the definition that

$$
\begin{aligned}
& X \subset \Lambda_{\theta}^{0} \cap \Lambda_{\theta}^{1} \subset X \cup Y, \\
& Y \subset \Lambda_{\nu}^{0} \cap \Lambda_{\nu}^{1} \subset X \cup Y .
\end{aligned}
$$

But in fact we have the following sharper statement:

Lemma 7.4. With the above notation, we have

$$
\Lambda_{\theta}^{0} \cap \Lambda_{\theta}^{1}=\Lambda_{\nu}^{0} \cap \Lambda_{\nu}^{1}=X \cup Y .
$$

Proof. Take a point $y \in Y$ and assume that $\tilde{B}_{\theta, \nu}^{\circ n}(y)=c$. By Lemma 6.7 there are exactly two drop-chains which land at the critical point $c$ from different sides of $\Sigma_{\nu}$. Then the pull-backs of these drop-chains along the orbit $y, \tilde{B}_{\theta, \nu}(y), \ldots, \tilde{B}_{\theta, \nu}^{\circ n}(y)=c$ give two drop-chains which land at $y$ from different sides of $\Sigma_{\nu}$. These drop-chains are clearly subsets of the compact set $K_{\theta, \nu}$. The fact that they land at $y$ from different sides of $\Sigma_{\nu}$ implies $y \in \Lambda_{\theta}^{0} \cap \Lambda_{\theta}^{1}$. The proof of the other equality is similar.

Corollary 7.5. With the above notation, we have

$$
\Lambda_{\theta}^{0}=\Lambda_{\nu}^{1} \quad \text { and } \quad \Lambda_{\theta}^{1}=\Lambda_{\nu}^{0} .
$$

Proof. Let $\widehat{\mathbb{C}} \backslash \Sigma=O_{1} \cup O_{2}$, where the $O_{i}$ are disjoint topological disks with $\Lambda_{\theta}^{0} \subset \bar{O}_{1}$ and $\Lambda_{\theta}^{1} \subset \bar{O}_{2}$. Taking the orientations on the sphere into account, we have $\Lambda_{\nu}^{1} \subset \bar{O}_{1}$ and $\Lambda_{\nu}^{0} \subset \bar{O}_{2}$. Since $\Lambda_{\theta}^{0} \cup \Lambda_{\theta}^{1}=\partial K_{\theta, \nu}=\partial K_{\theta, \nu}^{\infty}=\Lambda_{\nu}^{0} \cup \Lambda_{\nu}^{1}$ by Corollary 4.6 and $\Lambda_{\theta}^{0} \cap \Lambda_{\theta}^{1}=\Lambda_{\nu}^{0} \cap \Lambda_{\nu}^{1}$ by Lemma [.4 it follows that $\Lambda_{\theta}^{0}=\Lambda_{\nu}^{1}$ and $\Lambda_{\theta}^{1}=\Lambda_{\nu}^{0}$.

We can now define the itinerary(ies) of a point $p \in \partial K_{\theta, \nu}$ with respect to $\Sigma_{\theta}$ by looking at the points in the forward orbit of $p$ and deciding whether they belong to $\Lambda_{\theta}^{0}$ or $\Lambda_{\theta}^{1}$. However, we may face an ambiguity in defining the digits when some forward iterate of $p$ belongs to the intersection $\Lambda_{\theta}^{0} \cap \Lambda_{\theta}^{1}=X \cup Y$. This minor problem can be resolved in the same way we defined itineraries for the points in the Julia set $J\left(\tilde{Q}_{\theta}\right)$ (see subsection 7.1). For convenience, we explain this procedure more specifically. Let $p_{i}=\tilde{B}_{\theta, \nu}^{\circ i}(p)$ for $i \geq 0$. We distinguish four cases:

- Case 1. $p_{i} \notin X \cup Y$ for every $i \geq 0$. Then $p$ has a unique $\Sigma_{\theta}$-itinerary $\varepsilon_{\theta}=\left(\varepsilon_{0}, \varepsilon_{1}, \varepsilon_{2}, \ldots\right)$ and a unique $\Sigma_{\nu}$-itinerary $\varepsilon_{\nu}=\left(\delta_{0}, \delta_{1}, \delta_{2}, \ldots\right)$ determined by the condition $p_{i} \in \Lambda_{\theta}^{\varepsilon_{i}} \cap \Lambda_{\nu}^{\delta_{i}}$. It easily follows from Corollary 7.5 that $\delta_{i}=1-\varepsilon_{i}$ so that $\varepsilon_{\theta}$ and $\varepsilon_{\nu}$ have opposite digits.

- Case 2. There exists a smallest integer $n \geq 0$ such that $p_{n}=\beta$. If $n=0$ or 1 , i.e., if $p=\beta$ or $\beta^{\prime}$, the definition of itineraries is as follows:

$$
\begin{array}{ll}
\varepsilon_{\theta}(\beta)=\varepsilon_{\nu}^{\prime}(\beta)=(0,0,0, \ldots), & \varepsilon_{\theta}^{\prime}(\beta)=\varepsilon_{\nu}(\beta)=(1,1,1, \ldots), \\
\varepsilon_{\theta}\left(\beta^{\prime}\right)=\varepsilon_{\nu}^{\prime}\left(\beta^{\prime}\right)=(0,1,1, \ldots), & \varepsilon_{\theta}^{\prime}\left(\beta^{\prime}\right)=\varepsilon_{\nu}\left(\beta^{\prime}\right)=(1,0,0, \ldots) .
\end{array}
$$


Now let us assume $n \geq 2$. In this case, there are two $\Sigma_{\theta}$-itineraries

$$
\begin{aligned}
\varepsilon_{\theta} & =\left(\varepsilon_{0}, \varepsilon_{1}, \ldots, \varepsilon_{n-2}, 0,1,1,1, \ldots\right), \\
\varepsilon_{\theta}^{\prime} & =\left(\varepsilon_{0}, \varepsilon_{1}, \ldots, \varepsilon_{n-2}, 1,0,0,0, \ldots\right)
\end{aligned}
$$

and two $\Sigma_{\nu}$-itineraries

$$
\begin{aligned}
& \varepsilon_{\nu}=\left(\delta_{0}, \delta_{1}, \ldots, \delta_{n-2}, 1,0,0,0, \ldots\right), \\
& \varepsilon_{\nu}^{\prime}=\left(\delta_{0}, \delta_{1}, \ldots, \delta_{n-2}, 0,1,1,1, \ldots\right),
\end{aligned}
$$

where the initial segments are determined by the condition $p_{i} \in \Lambda_{\theta}^{\varepsilon_{i}} \cap \Lambda_{\nu}^{\delta_{i}}$ for $0 \leq i \leq n-2$. Again, note that the $\Sigma_{\theta^{-}}$and $\Sigma_{\nu}$-itineraries have opposite digits.

- Case 3. There exists a smallest integer $n \geq 0$ such that $p_{n} \in X \backslash\left\{\beta, \beta^{\prime}\right\}$. If $p_{n}=x_{11 \cdots 1} \in \tilde{B}_{\theta, \nu}^{-k+1}(1)$ for some $k \geq 1$, then $p$ has two $\Sigma_{\theta}$-itineraries

$$
\begin{aligned}
& \varepsilon_{\theta}=(\varepsilon_{0}, \varepsilon_{1}, \ldots, \varepsilon_{n-1}, \underbrace{0, \ldots, 0}_{k \text { terms }}, \underbrace{\omega_{1}, \omega_{2}, \ldots}_{\Sigma_{\theta} \text {-itinerary of } \tilde{B}_{\theta, \nu}(1)}), \\
& \varepsilon_{\theta}^{\prime}=(\varepsilon_{0}, \varepsilon_{1}, \ldots, \varepsilon_{n-1}, \underbrace{1, \ldots, 1}_{k \text { terms }}, \underbrace{\underbrace{\omega_{1} \omega_{2}, \ldots}_{1}}_{\Sigma_{\theta} \text {-itinerary of } \tilde{B}_{\theta, \nu}(1)})
\end{aligned}
$$

and two $\Sigma_{\nu}$-itineraries

$$
\begin{aligned}
& \varepsilon_{\nu}=(\delta_{0}, \delta_{1}, \ldots, \delta_{n-1}, \underbrace{1, \ldots, 1}_{k \text { terms }}, \underbrace{\sigma_{1}, \sigma_{2}, \ldots}_{\Sigma_{\nu} \text {-itinerary of } \tilde{B}_{\theta, \nu}(1)}), \\
& \varepsilon_{\nu}^{\prime}=(\delta_{0}, \delta_{1}, \ldots, \delta_{n-1}, \underbrace{0, \ldots, 0}_{k \text { terms }}, \underbrace{\underbrace{}_{1}, \sigma_{2}, \ldots}_{\Sigma_{\nu} \text {-itinerary of } \tilde{B}_{\theta, \nu}(1)}) .
\end{aligned}
$$

Here the initial segments and the itineraries of $\tilde{B}_{\theta, \nu}(1)$ are uniquely determined by Case 1 (the initial segments are empty if $n=0$ ). If, on the other hand, $p_{n}=$ $x_{211 \cdots 1} \in \tilde{B}_{\theta, \nu}^{-k}(1)$ for some $k \geq 1$, then $p$ has two $\Sigma_{\theta}$-itineraries

$$
\begin{aligned}
& \varepsilon_{\theta}=(\varepsilon_{0}, \varepsilon_{1}, \ldots, \varepsilon_{n-1}, 0, \underbrace{1, \ldots, 1}_{k \text { terms }}, \underbrace{\omega_{1}, \omega_{2}, \ldots}_{\Sigma_{\theta} \text {-itinerary of } \tilde{B}_{\theta, \nu}(1)}), \\
& \varepsilon_{\theta}^{\prime}=(\varepsilon_{0}, \varepsilon_{1}, \ldots, \varepsilon_{n-1}, 1, \underbrace{0, \ldots, 0}_{k \text { terms }}, \underbrace{\omega_{1}, \omega_{2}, \ldots}_{\Sigma_{\theta} \text {-itinerary of } \tilde{B}_{\theta, \nu}(1)})
\end{aligned}
$$

and two $\Sigma_{\nu}$-itineraries

$$
\begin{aligned}
& \varepsilon_{\nu}=(\delta_{0}, \delta_{1}, \ldots, \delta_{n-1}, 1, \underbrace{0, \ldots, 0}_{k \text { terms }}, \underbrace{\sigma_{1}, \sigma_{2}, \ldots}_{\Sigma_{\nu} \text {-itinerary of } \tilde{B}_{\theta, \nu}(1)}), \\
& \varepsilon_{\nu}^{\prime}=(\delta_{0}, \delta_{1}, \ldots, \delta_{n-1}, 0, \underbrace{1, \ldots, 1}_{k \text { terms }}, \underbrace{\sigma_{1}, \sigma_{2}, \ldots}_{\Sigma_{\nu} \text {-itinerary of } \tilde{B}_{\theta, \nu}(1)}) .
\end{aligned}
$$

- Case 4. There exists a smallest integer $n \geq 0$ such that $p_{n} \in Y \backslash\left\{\beta, \beta^{\prime}\right\}$. This case is similar to Case 3 by switching $\theta \leftrightarrow \nu$ and $1 \leftrightarrow c$. 
We summarize the above construction in the following

Proposition 7.6 (Two or four itineraries). Let $p \in \partial K_{\theta, \nu}$. Then, either $p$ is not a preimage of $\beta, 1$ or $c$ in which case it has a unique $\Sigma_{\theta}$-itinerary $\varepsilon_{\theta}$ and a unique $\Sigma_{\nu}$-itinerary $\varepsilon_{\nu}$, or $p$ is a preimage of $\beta, 1$ or $c$ in which case it has two different $\Sigma_{\theta}$-itineraries $\varepsilon_{\theta}, \varepsilon_{\theta}^{\prime}$ and two different $\Sigma_{\nu}$-itineraries $\varepsilon_{\nu}, \varepsilon_{\nu}^{\prime}$.

The following is a straightforward consequence of the above construction as well as Corollary 7.5

Proposition $7.7\left(\Sigma_{\theta^{-}}\right.$and $\Sigma_{\nu}$-itineraries have opposite digits). Let $p \in \partial K_{\theta, \nu}$ have $\Sigma_{\theta}$-itinerary $\varepsilon_{\theta}(p)$. Then the $\Sigma_{\nu}$-itinerary $\varepsilon_{\nu}(p)$ of $p$ is obtained by converting all 0 's to 1 's and all 1 's to 0 's in $\varepsilon_{\theta}(p)$. In other words, $\varepsilon_{\nu}(p)=\mathbf{1}-\varepsilon_{\theta}(p)$, where $\mathbf{1}=(1,1,1, \ldots)$. In the case where $p$ has two itineraries, we have $\varepsilon_{\nu}(p)=\mathbf{1}-\varepsilon_{\theta}(p)$ and $\varepsilon_{\nu}^{\prime}(p)=\mathbf{1}-\varepsilon_{\theta}^{\prime}(p)$.

The following lemma also follows from the above construction and subsection 7.1 .

Lemma 7.8 (Itineraries match). Let $z \in \partial K_{\theta}$ and $p=\zeta_{\theta}(z) \in \partial K_{\theta, \nu}$.

(i) Suppose that $z$ is not a preimage of the fixed point $\beta$ or the critical point 1 for $\tilde{Q}_{\theta}$. Then the unique itinerary of $z$ with respect to $S_{\theta}$ coincides with $\varepsilon_{\theta}(p)$ when $p$ is not a preimage of $c$, and it coincides with one of the two itineraries $\varepsilon_{\theta}(p)$ or $\varepsilon_{\theta}^{\prime}(p)$ when $p$ is a preimage of $c$.

(ii) Suppose that $z$ is a preimage of the fixed point $\beta$ or the critical point 1 for $\tilde{Q}_{\theta}$. Then the two itineraries of $z$ with respect to $S_{\theta}$ coincide with the two itineraries $\varepsilon_{\theta}(p)$ and $\varepsilon_{\theta}^{\prime}(p)$.

Similar statements hold when $z \in \partial K_{\nu}$ and $p=\zeta_{\nu}(z)$.

Corollary 7.9 (Itineraries determine points). Two points in $\partial K_{\theta, \nu}$ with the same $\Sigma_{\theta}$ - or $\Sigma_{\nu}$-itinerary must coincide.

Proof. Let $p, q \in \partial K_{\theta, \nu}$ and assume for example that $\varepsilon_{\theta}(p)=\varepsilon_{\theta}(q)$. When $p$ (hence $q$ ) is a preimage of $\beta, 1$ or $c$, it is easy to see that identical $\Sigma_{\theta}$-itineraries implies $p=q$. So let us assume that $p$ and $q$ are not preimages of $\beta, 1$ or $c$. Since $\zeta_{\theta}: K_{\theta} \rightarrow K_{\theta, \nu}$ is surjective, we have $p=\zeta_{\theta}(u)$ and $q=\zeta_{\theta}(v)$ for some $u, v \in \partial K_{\theta}=J\left(\tilde{Q}_{\theta}\right)$. By Lemma $7.8(\mathrm{i}), u$ and $v$ have the same itineraries with respect to $S_{\theta}$. By Proposition 7.1 (i), $u=v$. Hence $p=q$.

Now we are ready to finish the proof of Theorem 7.2 Consider two points $z \in \partial K_{\theta}$ and $w \in \partial K_{\nu}$ such that $z=\eta_{\theta}(t)$ and $w=\eta_{\nu}(-t)$ for some $t \in \mathbb{T}$. Set $p=\zeta_{\theta}(z)$ and $q=\zeta_{\nu}(w)$. The binary digits $\left(\varepsilon_{0}, \varepsilon_{1}, \varepsilon_{2}, \ldots\right)$ of the angle $t$ form an itinerary of $z$ with respect to $S_{\theta}$. Since $t=0 . \varepsilon_{0} \varepsilon_{1} \varepsilon_{2} \ldots$ in base $2,-t$ has the binary expansion $0 . \delta_{0} \delta_{1} \delta_{2} \ldots$, where $\delta_{i}=1-\varepsilon_{i}$. Hence $\left(\delta_{0}, \delta_{1}, \delta_{2}, \ldots\right)$ is an itinerary of $w$ with respect to $S_{\nu}$. Thus by Lemma [7.8 $\left(\varepsilon_{0}, \varepsilon_{1}, \varepsilon_{2}, \ldots\right)=\varepsilon_{\theta}(p)$ (or possibly $\left.\varepsilon_{\theta}^{\prime}(p)\right)$ and $\left(\delta_{0}, \delta_{1}, \delta_{2}, \ldots\right)=\varepsilon_{\nu}(q)$ (or possibly $\varepsilon_{\nu}^{\prime}(q)$ ). By Proposition 7.7 $\left(\varepsilon_{0}, \varepsilon_{1}, \varepsilon_{2}, \ldots\right)=\varepsilon_{\theta}(q)$ (or possibly $\varepsilon_{\theta}^{\prime}(q)$ ), which means $p$ and $q$ have the same $\Sigma_{\theta}$-itinerary. This, by Corollary 7.9 implies $p=q$.

Conversely, assume that $\zeta_{\theta}(z)=\zeta_{\nu}(w)=p$. We consider three cases: First assume that $p$ is not a preimage of $\beta, 1$ or $c$. Then it follows from Proposition [7.7 that $\varepsilon_{\theta}(p)=\mathbf{1}-\varepsilon_{\nu}(p)=\left(\varepsilon_{0}, \varepsilon_{1}, \varepsilon_{2}, \ldots\right)$ and these itineraries are unique. By Lemma [7.8, $\left(\varepsilon_{0}, \varepsilon_{1}, \varepsilon_{2}, \ldots\right)$ is the $S_{\theta}$-itinerary of $z$ and $\left(1-\varepsilon_{0}, 1-\varepsilon_{1}, 1-\varepsilon_{2}, \ldots\right)$ 
is the $S_{\nu}$-itinerary of $w$. Setting $t=0 . \varepsilon_{0} \varepsilon_{1} \varepsilon_{2} \ldots$ in base 2 , we have $z=\eta_{\theta}(t)$ and $w=\eta_{\nu}(-t)$ and we are done. Next, assume that $p$ is a preimage of $\beta$. Then both $z$ and $w$ are preimages of the corresponding $\beta$-fixed points for $\tilde{Q}_{\theta}$ and $\tilde{Q}_{\nu}$. By Lemma 7.8 the two $\Sigma_{\theta}$-itineraries of $p$ coincide with those of $z$ with respect to $S_{\theta}$, both of which determine the same angle $t$ with $z=\eta_{\theta}(t)$. Similarly, the two $\Sigma_{\nu^{-}}$ itineraries of $p$ coincide with those of $w$ with respect to $S_{\nu}$, both of which determine the same angle $s$ with $w=\eta_{\nu}(s)$. But $\varepsilon_{\nu}(p)=\mathbf{1}-\varepsilon_{\theta}(p)$ and $\varepsilon_{\nu}^{\prime}(p)=\mathbf{1}-\varepsilon_{\theta}^{\prime}(p)$, which implies the binary digits of $t$ and $s$ are opposite, so $t=-s$. Finally, assume that $p$ is a preimage of, say, 1 . Then, as 1 and $c$ have disjoint orbits under $\tilde{B}_{\theta, \nu}$, $p$ cannot be a preimage of $c$. This implies that $z$ is a preimage of the critical point 1 of $\tilde{Q}_{\theta}$ and therefore has two $S_{\theta}$-itineraries, and $w$ is not a preimage of the $\beta$-fixed point or the critical point 1 of $\tilde{Q}_{\nu}$ and so has a unique $S_{\nu}$-itinerary. Let $w=\eta_{\nu}(-t)$, where the unique $t \in \mathbb{T}$ has binary expansion $t=0 \varepsilon_{0} \varepsilon_{1} \varepsilon_{2} \ldots$ By Lemma 7.8. $\left(1-\varepsilon_{0}, 1-\varepsilon_{1}, 1-\varepsilon_{2}, \ldots\right)$ is one of the $\Sigma_{\nu}$-itineraries of $p$. Hence by Proposition 7.7, $\left(\varepsilon_{0}, \varepsilon_{1}, \varepsilon_{2}, \ldots\right)$ is one of the $\Sigma_{\theta}$-itineraries of $p$. Therefore, by another application of Lemma 7.8, $\left(\varepsilon_{0}, \varepsilon_{1}, \varepsilon_{2}, \ldots\right)$ is one of the two $S_{\theta}$-itineraries of $z$, implying $z=\eta_{\theta}(t)$.

This covers all the cases and completes the proof of Theorem 7.2

We conclude with the following:

Corollary 7.10 (At most three points). Let $p \in \partial K_{\theta, \nu}$. Then $\zeta_{\theta}^{-1}(p) \cup \zeta_{\nu}^{-1}(p)$ contains at most three points.

Proof. Since $p$ has at most two $\Sigma_{\theta}$-itineraries and two $\Sigma_{\nu}$-itineraries, Lemma 7.8 and Proposition 7.1 imply that $\zeta_{\theta}^{-1}(p)$ and $\zeta_{\nu}^{-1}(p)$ each contain at most two points. So to prove the corollary, we assume by way of contradiction that there are four distinct points $z_{1}, z_{2} \in \partial K_{\theta}$ and $z_{3}, z_{4} \in \partial K_{\nu}$ such that $\zeta_{\theta}\left(z_{1}\right)=\zeta_{\theta}\left(z_{2}\right)=\zeta_{\nu}\left(z_{3}\right)=$ $\zeta_{\nu}\left(z_{4}\right)=p$. By Theorem 7.2, all four points have to be biaccessible. Pick, for example, $z_{1}$ and $z_{3}$ and note that they eventually map to the critical points of $\tilde{Q}_{\nu}$ and $\tilde{Q}_{\theta}$ [Za1]. Hence $p=\zeta_{\theta}\left(z_{1}\right)$ eventually maps to the critical point 1 of $\tilde{B}_{\theta, \nu}$ and $p=\zeta_{\nu}\left(z_{3}\right)$ also maps to the critical point $c$ of $\tilde{B}_{\theta, \nu}$. This is clearly impossible since $c$ and 1 have disjoint orbits.

7.3. End of the proof. We can now prove the main theorem of this paper:

Theorem 7.11 (Bounded type Siegel quadratics are mateable). Let $0<\theta, \nu<1$ be two irrationals of bounded type and $\theta \neq 1-\nu$. Then the quadratic polynomials $f_{\theta}$ and $f_{\nu}$ are topologically mateable. Moreover, there exists a quadratic rational map $F$ such that $F=f_{\theta} \sqcup f_{\nu}$. Any two such rational maps are conjugate by a Möbius transformation.

Proof. The last assertion is immediate since every quadratic rational map with two fixed Siegel disks of rotation numbers $\theta$ and $\nu$ is holomorphically conjugate to the normalized map $F_{\theta, \nu}$ defined in (2.1). By Definition IIa of the introduction, it suffices to construct continuous maps $\varphi_{\theta}: K\left(f_{\theta}\right) \rightarrow \widehat{\mathbb{C}}$ and $\varphi_{\nu}: K\left(f_{\nu}\right) \rightarrow \widehat{\mathbb{C}}$ with the following properties:

(a) $\varphi_{\theta} \circ f_{\theta}=F_{\theta, \nu} \circ \varphi_{\theta}$ and $\varphi_{\nu} \circ f_{\nu}=F_{\theta, \nu} \circ \varphi_{\nu}$.

(b) $\varphi_{\theta}\left(K\left(f_{\theta}\right)\right) \cup \varphi_{\nu}\left(K\left(f_{\nu}\right)\right)=\widehat{\mathbb{C}}$.

(c) $\varphi_{\theta}$ and $\varphi_{\nu}$ are conformal in the interiors of $K\left(f_{\theta}\right)$ and $K\left(f_{\nu}\right)$.

(d) $\varphi_{\theta}(z)=\varphi_{\nu}(w)$ if and only if $z$ and $w$ are ray equivalent. 
It is clear from the preceding discussion what these maps should be. By the surgery construction of subsections 3.5 and 4.2, there exist quasiconformal homeomorphisms $\psi_{\theta}, \psi_{\nu}, \psi: \widehat{\mathbb{C}} \rightarrow \widehat{\mathbb{C}}$ such that

$$
\begin{aligned}
\psi_{\theta} \circ \tilde{Q}_{\theta} & =f_{\theta} \circ \psi_{\theta}, \\
\psi_{\nu} \circ \tilde{Q}_{\nu} & =f_{\nu} \circ \psi_{\nu}, \\
\psi \circ \tilde{B}_{\theta, \nu} & =F_{\theta, \nu} \circ \psi .
\end{aligned}
$$

Consider the semiconjugacies $\zeta_{\theta}$ and $\zeta_{\nu}$ of Theorem 7.2 and define

$$
\begin{aligned}
& \varphi_{\theta}=\psi \circ \zeta_{\theta} \circ \psi_{\theta}^{-1}, \\
& \varphi_{\nu}=\psi \circ \zeta_{\nu} \circ \psi_{\nu}^{-1} .
\end{aligned}
$$

Properties (a) and (b) above are immediate consequences of the corresponding properties of $\zeta_{\theta}$ and $\zeta_{\nu}$ stated in Theorem 7.2. So to finish the proof, we must show (c) and $(\mathrm{d})$.

To show (c), recall the surgery construction of subsection 3.5. Consider the Douady-Earle extension $H_{\theta}$ used in defining the modified Blaschke product $\tilde{Q}_{\theta}$ in (3.2). The invariant conformal structure $\sigma_{\theta}$ on the unit disk $\mathbb{D}$ is given by the pull-back of the standard conformal structure $\sigma_{0}$ under $H_{\theta}$. Similarly, we have the Douady-Earle extension $H_{\theta, \nu}$ for the linearizing homeomorphism of $B_{\theta, \nu}: \mathbb{T} \rightarrow \mathbb{T}$ used in defining the modified Blaschke product $\tilde{B}_{\theta, \nu}$ in (4.9), and the invariant conformal structure $\sigma_{\theta, \nu}$ on $\mathbb{D}$ as the pull-back of $\sigma_{0}$ under $H_{\theta, \nu}$. Both $H_{\theta}$ and $H_{\theta, \nu}$ conjugate $\tilde{Q}_{\theta}$ and $\tilde{B}_{\theta, \nu}$ to the rigid rotation $z \mapsto e^{2 \pi i \theta} z$. By definition of $\zeta_{\theta}$, we have $\zeta_{\theta}=H_{\theta, \nu}^{-1} \circ H_{\theta}$ on $\mathbb{D}$. This means that $\zeta_{\theta}$ pulls $\sigma_{\theta, \nu}$ back to $\sigma_{\theta}$ on the unit disk. It follows that the composition $\varphi_{\theta}=\psi \circ \zeta_{\theta} \circ \psi_{\theta}^{-1}$ on $\mathbb{D}$ pulls $\sigma_{0}$ back to $\sigma_{0}$, hence it is conformal there. Then (a) and the fact that $f_{\theta}$ and $F_{\theta, \nu}$ are holomorphic show that $\zeta_{\theta}$ is conformal in the interior of $K\left(f_{\theta}\right)$. A similar argument applies to $\zeta_{\nu}$.

To show (d), we note that the quasiconformal conjugacies $\psi_{\theta}$ and $\psi_{\nu}$ are conformal outside the filled Julia sets, so they preserve the external angles. Therefore $\gamma_{\theta}=\psi_{\theta} \circ \eta_{\theta}$ and $\gamma_{\nu}=\psi_{\nu} \circ \eta_{\nu}$, where $\gamma_{\theta}$ and $\gamma_{\nu}$ are the Carathéodory loops of $J\left(f_{\theta}\right)$ and $J\left(f_{\nu}\right)$. By Theorem[7.2 $\varphi_{\theta}(z)=\varphi_{\nu}(w)$ implies that $z=\gamma_{\theta}(t)$ and $w=\gamma_{\nu}(-t)$ for some $t \in \mathbb{T}$, which means $z$ and $w$ are ray equivalent. The converse statement is almost immediate because if $z \in K\left(f_{\theta}\right)$ is ray equivalent to $w \in K\left(f_{\nu}\right)$, the same is true for $\psi_{\theta}^{-1}(z)$ and $\psi_{\nu}^{-1}(w)$. Since every pair of ray equivalent points of the form $\left(\eta_{\theta}(t), \eta_{\nu}(-t)\right)$ is mapped to the same point under $\left(\zeta_{\theta}, \zeta_{\nu}\right)$, the same must be true for arbitrary pairs of ray equivalent points. Hence $\zeta_{\theta}\left(\psi_{\theta}^{-1}(z)\right)=\zeta_{\nu}\left(\psi_{\nu}^{-1}(w)\right)$, or $\varphi_{\theta}(z)=\varphi_{\nu}(w)$. This proves $(\mathrm{d})$, and finishes the proof of the Main Theorem 7.11 .

\section{Concluding Remarks}

In this section, we discuss some corollaries of Theorem 7.11 In particular, we describe the nature of the pinch points already observed in Figure 2 Then we prove a number-theoretic corollary of the topological mateability part of Theorem 7.11 which is related to the rotation sets of the angle-doubling map on the circle. Finally, we conclude with a discussion of the special case of a self-mating $f_{\theta} \sqcup f_{\theta}$ and mating $f_{\theta}$ with the Chebyshev polynomial $z \mapsto z^{2}-2$.

8.1. Ray equivalence classes and pinch points. Consider two irrationals $\theta$ and $\nu$ of bounded type, with $\theta \neq 1-\nu$, the quadratic polynomials $f_{\theta}$ and $f_{\nu}$, and the 
rational map $F_{\theta, \nu}$. Let

$$
K\left(F_{\theta, \nu}\right)=\left\{z \in \mathbb{C}: \text { the orbit }\left\{F_{\theta, \nu}^{\circ n}(z)\right\}_{n \geq 0} \text { never intersects } \Delta^{\infty}\right\},
$$

and similarly

$$
K^{\infty}\left(F_{\theta, \nu}\right)=\left\{z \in \mathbb{C}: \text { the orbit }\left\{F_{\theta, \nu}^{\circ n}(z)\right\}_{n \geq 0} \text { never intersects } \Delta^{0}\right\} .
$$

(In Figure 2 these two sets are the compact sets in black and gray respectively.) As we have already noted in the introduction, $K\left(F_{\theta, \nu}\right)$ is not a full set. In fact, it is evident from Figure 2 that there are infinitely many identifications between pairs of landing points of drop-chains in $K\left(F_{\theta, \nu}\right)$ which correspond to the pinch points of $K^{\infty}\left(F_{\theta, \nu}\right)$, namely the preimages of the critical point $c \in \partial \Delta^{\infty}$. A similar fact holds for drop-chains of $K^{\infty}\left(F_{\theta, \nu}\right)$ and the pinch points of $K\left(F_{\theta, \nu}\right)$. We gave a precise version of this statement in Lemma 6.7 It follows that every precritical point in the Julia set of $f_{\theta}$ (resp. $f_{\nu}$ ) is identified with the landing points of two distinct drop-chains of $f_{\nu}$ (resp. $f_{\theta}$ ). Theorem 7.11 allows us to determine exactly which two drop-chains correspond to the given pinch point. Throughout the following discussion, we continue using notations from $\$ 7$.

Recall that the quasiconformal conjugacies $\psi_{\theta}$ (between $\tilde{Q}_{\theta}$ and $f_{\theta}$ ) and $\psi_{\nu}$ (between $\tilde{Q}_{\nu}$ and $f_{\nu}$ ) in (7.3) are conformal in the basins of infinity, so they preserve the ray equivalence classes. From this fact and Corollary 7.10, it follows that for the formal mating of $f_{\theta}$ and $f_{\nu}$, every ray equivalence class intersects $K\left(f_{\theta}\right) \cup K\left(f_{\nu}\right)$ in at most three points. Let $E$ denote the intersection of a ray equivalence class with the union $K\left(f_{\theta}\right) \cup K\left(f_{\nu}\right)$. We only have three possibilities for $E$ :

- Case 1. $E=\{z, w\}$, where $z \in K\left(f_{\theta}\right)$ and $w \in K\left(f_{\nu}\right)$ are both the landing points of unique rays, hence $z=\gamma_{\theta}(t)$ and $w=\gamma_{\nu}(-t)$ for a unique $t \in \mathbb{T}$.

- Case 2. $E=\left\{z, z^{\prime}, w\right\}$, where $z, z^{\prime} \in K\left(f_{\theta}\right)$ are both the landing points of unique rays and $w \in K\left(f_{\nu}\right)$ is biaccessible, hence a preimage of the critical point of $f_{\nu}$. In this case, there exist $s, t \in \mathbb{T}$ such that $z=\gamma_{\theta}(s), z^{\prime}=\gamma_{\theta}(t)$, and $w=\gamma_{\nu}(-s)=\gamma_{\nu}(-t)$.

- Case 3. $E=\left\{z, w, w^{\prime}\right\}$, where $z \in K\left(f_{\theta}\right)$ is biaccessible, and $w, w^{\prime} \in K\left(f_{\nu}\right)$ are both the landing points of unique rays. In this case, there exist $s, t \in \mathbb{T}$ such that $z=\gamma_{\theta}(s)=\gamma_{\theta}(t), w=\gamma_{\nu}(-t), w^{\prime}=\gamma_{\nu}(-s)$.

Corollary 8.1 (Pinch points in $K\left(F_{\theta, \nu}\right)$ ). The compact set $K\left(F_{\theta, \nu}\right)$ is homeomorphic to the quotient of the filled Julia set $K\left(f_{\theta}\right)$ by an equivalence relation $\sim$ defined as follows. Two points $z \neq z^{\prime}$ in $K\left(f_{\theta}\right)$ satisfy $z \sim z^{\prime}$ if and only if they are the landing points of unique rays at angles $s, t \in \mathbb{T}, z=\gamma_{\theta}(s), z^{\prime}=\gamma_{\theta}(t)$, such that $\gamma_{\nu}(-s)=\gamma_{\nu}(-t)$. Every non-trivial equivalence class of $\sim$ contains exactly two points which are necessarily the landing points of two distinct drop-chains of $f_{\theta}$.

Proof. Since $\varphi_{\theta}: K\left(f_{\theta}\right) \rightarrow K\left(F_{\theta, \nu}\right)$ is a surjective map, $K\left(F_{\theta, \nu}\right)$ is homeomorphic to $K\left(f_{\theta}\right) / \sim$, where $z \sim z^{\prime}$ if and only if $z$ and $z^{\prime}$ belong to the same fiber of $\varphi_{\theta}$. By Case 2 of the above discussion, for distinct points $z \neq z^{\prime}$, we have $\varphi_{\theta}(z)=\varphi_{\theta}\left(z^{\prime}\right)$ if and only if there exist $w \in K\left(f_{\nu}\right)$ and distinct angles $s, t \in \mathbb{T}$ such that $z=\gamma_{\theta}(s)$, $z^{\prime}=\gamma_{\theta}(t)$, and $w=\gamma_{\nu}(-s)=\gamma_{\nu}(-t)$. In this case $w$ is a preimage of the critical point of $f_{\nu}$. Both $z$ and $z^{\prime}$ are landing points of distinct drop-chains of $f_{\theta}$, for otherwise $z$ or $z^{\prime}$ would belong to the closure of a drop (Proposition [3.8), hence $\varphi_{\theta}(z)=\varphi_{\theta}\left(z^{\prime}\right)$ would eventually map to the boundary of the Siegel disk $\Delta^{0}$ of $F_{\theta, \nu}$. On the other hand, $\varphi_{\theta}(z)=\varphi_{\nu}(w)$ eventually maps to the critical point of $F_{\theta, \nu}$ on the boundary of $\Delta^{\infty}$. This would contradict $\partial \Delta^{0} \cap \partial \Delta^{\infty}=\emptyset$. 
This completely describes which identifications are made in $K\left(f_{\theta}\right)$ in order to obtain $K\left(F_{\theta, \nu}\right)$ : Take any precritical point in the Julia set of $f_{\nu}$ and calculate the angles $s, t$ of the two external rays landing on it. Then find the landing points of the external rays at angles $-s$ and $-t$ for $f_{\theta}$, which are ends of distinct drop-chains, and identify them in $K\left(f_{\theta}\right)$. This creates a "pinch point". After all such possible identifications are made, we obtain a homeomorphic copy of $K\left(F_{\theta, \nu}\right)$. Note that not all the landing points of drop-chains of $f_{\theta}$ undergo this identification, simply because there are uncountably many drop-chains and only countably many pinch points.

8.2. Rotation sets of the doubling map. The angle $\omega=\omega(\theta)$ of the external ray landing at the critical value of the quadratic polynomial $f_{\theta}$ may be described in terms of the rotation sets of the angle-doubling map on $\mathbb{T}$ defined by $m_{2}: x \mapsto 2 x$ $(\bmod 1)$. A subset $E \subset \mathbb{T}$ is called a rotation set if the restriction of $m_{2}$ to $E$ is order-preserving, with $m_{2}(E) \subset E$. It is easy to see that in this case $E$ must be contained in a closed semicircle. Hence the restriction $\left.m_{2}\right|_{E}$ can be extended to a degree 1 monotone map of the circle, which has a well-defined rotation number, denoted by $\rho(E) \in[0,1)$. The following theorem can be found in $[\mathrm{A}]$ and $[\overline{\mathrm{BS}}]$ :

Theorem 8.2 (Rotation sets of the doubling map). (i) For any $0 \leq \theta<1$ there exists a unique compact rotation set $E_{\theta} \subset \mathbb{T}$ with $\rho\left(E_{\theta}\right)=\theta$. When $\theta$ is rational $E_{\theta}$ is a single periodic orbit of $m_{2}$. On the other hand, when $\theta$ is irrational, $E_{\theta}$ is a Cantor set contained in a well-defined semicircle $[\omega / 2$, $(\omega+1) / 2]$, with $\{\omega / 2,(\omega+1) / 2\} \subset E_{\theta}$, and the action of $m_{2}$ on $E_{\theta}$ is minimal. In this case the angle $\omega=\omega(\theta)$ can be computed in terms of $\theta$ as

$$
\omega=\sum_{0<p / q<\theta} 2^{-q},
$$

where the sum is taken over all (not necessarily reduced) fractions $p / q$.

(ii) For every $0<\omega<1$, the semicircle $[\omega / 2,(\omega+1) / 2]$ contains a unique compact minimal rotation set $E^{\omega}$. The graph of $\omega \mapsto \rho\left(E^{\omega}\right)$ is a devil's staircase.

The mapping $\omega \mapsto \rho\left(E^{\omega}\right)$ is intimately connected with the parameter rays defining the limbs of the Mandelbrot set $\mathrm{A}]$.

Now consider the quadratic polynomial $f_{\theta}$ for an irrational $\theta$ of bounded type. Then the Julia set $J\left(f_{\theta}\right)$ is locally-connected, and the boundary of the Siegel disk $\Delta$ of $f_{\theta}$ is a quasicircle passing through the critical point 0 (compare Theorem 3.5 and Theorem 3.10). We know that 0 is the landing point of exactly two external rays at angles $\omega / 2$ and $(\omega+1) / 2$, where $0<\omega<1$. Define

$$
E=\left\{t \in \mathbb{T}: \gamma_{\theta}(t) \in \partial \Delta\right\}
$$

It is easy to see that $E$ is compact and contained in the semicircle $[\omega / 2,(\omega+1) / 2]$, hence by the above theorem, $E=E^{\omega}$. On the other hand, the order of the points in the orbit $\left\{f_{\theta}^{\circ n}(0)\right\}_{n \geq 0}$ on the boundary $\partial \Delta$ determines the rotation number $\theta$ uniquely dMvS]. At the same time this order coincides with the order of the orbit of $\omega$ under $m_{2}$ on the circle. It follows that $\rho\left(E^{\omega}\right)=\theta$.

Corollary 8.3. When $0<\theta<1$ is an irrational of bounded type, the angle $0<$ $\omega(\theta)<1$ of the external ray landing at the critical value of the quadratic polynomial $f_{\theta}$ is given by (8.1). 
It is interesting to investigate number-theoretic properties of the numbers $\omega(\theta)$ when $\theta$ is irrational. For example, it follows from the above discussion that for irrational $0<\theta<1, \omega(\theta)$ is also irrational. When $\theta$ is of bounded type, we have the much sharper statement that $\omega(\theta)$ is not $(2+(\sqrt{5}-1) / 2-\delta)$-Diophantine for any $\delta>0[\mathrm{BS}$. In particular, by Roth's theorem, $\omega(\theta)$ is transcendental over $\mathbb{Q}$. The topological mateability part of Theorem 7.11 allows us to draw a further conclusion:

Theorem 8.4. Suppose that $0<\theta, \nu<1$ are irrationals of bounded type, with $\theta \neq 1-\nu$, and consider the angles $\omega(\theta)$ and $\omega(\nu)$. Then the equation

$$
2^{n} \omega(\theta)+2^{m} \omega(\nu) \equiv 0(\bmod 1)
$$

does not have any solution in non-negative integers $n, m$.

Note that the condition $\theta \neq 1-\nu$ is necessary because $\omega(\theta)+\omega(1-\theta)=1$. Also, when $\theta=\nu$ the theorem follows from Theorem 8.2 simply because $\omega(\theta)$ is irrational.

Proof. Suppose that (8.2) holds for some $n, m$. Set $t=\omega(\theta) / 2^{m}$, so that $-2^{n+m} t \equiv$ $2^{m} \omega(\nu)(\bmod 1)$. Let $z=\gamma_{\theta}(t) \in J\left(f_{\theta}\right)$ and $w=\gamma_{\nu}(-t) \in J\left(f_{\nu}\right)$. Then $f_{\theta}^{\circ m}(z)=c_{\theta}$ is the critical value of $f_{\theta}$ and $f_{\nu}^{\circ n+m}(w)=f_{\nu}^{\circ m}\left(c_{\nu}\right)$ belongs to the forward orbit of the critical point of $f_{\nu}$. By Theorem 7.11, $F_{\theta, \nu}=f_{\theta} \sqcup f_{\nu}$, so $\varphi_{\theta}(z) \in J\left(F_{\theta, \nu}\right)$ and $\varphi_{\nu}(w) \in J\left(F_{\theta, \nu}\right)$ eventually hit $\partial \Delta^{0}$ and $\partial \Delta^{\infty}$, respectively. But $z$ and $w$ are ray equivalent, so $\varphi_{\theta}(z)=\varphi_{\nu}(w)$ by Theorem 7.11 This contradicts $\partial \Delta^{0} \cap \partial \Delta^{\infty}=$ $\emptyset$.

8.3. Mating with the Chebyshev quadratic polynomial. When $\theta=\nu$, the self-mating $F=F_{\theta, \theta}=f_{\theta} \sqcup f_{\theta}$ given by Theorem 7.11 has a natural symmetry, i.e., it commutes with the involution $\mathcal{I}: z \mapsto 1 / z$ of the sphere. As was apparently first observed by Petersen, if we destroy this symmetry by passing to the quotient space, we can create new examples of mating.

Consider the quotient of the Riemann sphere by the action of $\mathcal{I}$. The resulting space is again a Riemann surface conformally isomorphic to the sphere $\widehat{\mathbb{C}}$. Since $F \circ \mathcal{I}=\mathcal{I} \circ F$, there is a well-defined rational map $G$ which makes the following diagram commute:

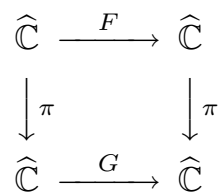

Here $\pi: \widehat{\mathbb{C}} \rightarrow \widehat{\mathbb{C}} / \mathcal{I} \simeq \widehat{\mathbb{C}}$ is the degree 2 natural projection. Chasing around this diagram shows that $G$ is a quadratic rational map which clearly has one Siegel disk of rotation number $\theta$. Therefore this way of collapsing the sphere identifies the two critical points of $F$ but creates a new critical point of its own. It is not hard to check that $G$ is Möbius conjugate to the map

$$
z \mapsto \frac{4 z}{\left((1+z)+e^{2 \pi i \theta}(1-z)\right)^{2}},
$$

with a fixed Siegel disk centered at 1 . The critical point $c_{1}=\left(e^{2 \pi i \theta}+1\right) /\left(e^{2 \pi i \theta}-1\right)$ of this map has the finite orbit $c_{1} \mapsto \infty \mapsto 0$. The second critical point $c_{2}=-c_{1}$ belongs to the boundary of the Siegel disk (compare Figure 14). 


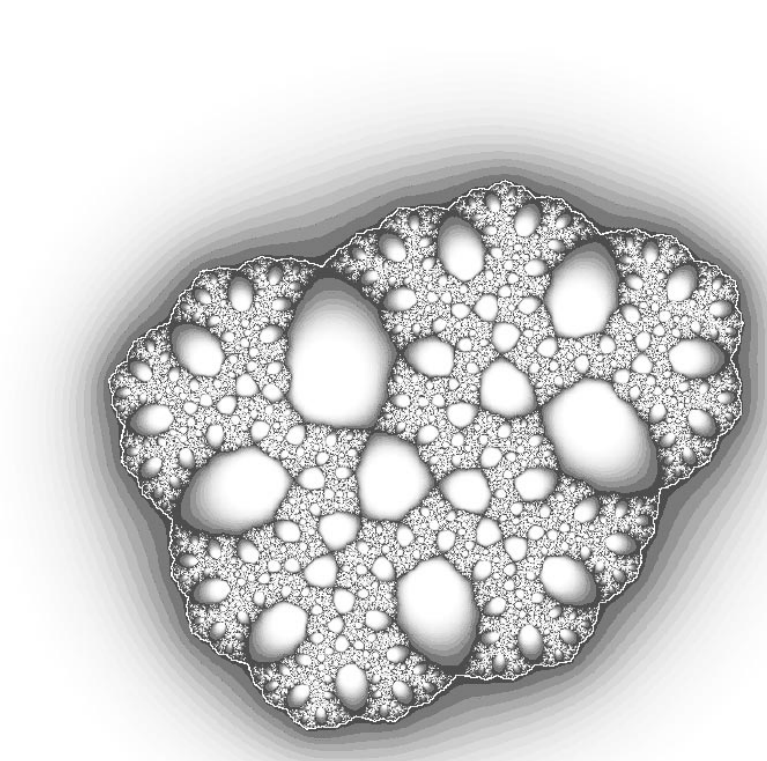

Figure 14. The Julia set of the mating $f_{\theta} \sqcup f_{\text {cheb }}$, where $\theta=$ $(\sqrt{5}-1) / 2$. To get a better picture we have conjugated the map in (8.3) by $w=1 /(z-1)$ so as to put the center of the Siegel disk at infinity and the finite critical orbit at $\left(e^{2 \pi i \theta}+1\right) / 2 \mapsto 0 \mapsto-1$.

Recall that the Chebyshev quadratic polynomial is $f_{\text {cheb }}: z \mapsto z^{2}-2$. It is easy to see that the filled Julia set $K\left(f_{\text {cheb }}\right)=J\left(f_{\text {cheb }}\right)$ is the closed interval $[-2,2]$. Its Carathéodory loop $\gamma_{\text {cheb }}: \mathbb{T} \rightarrow J\left(f_{\text {cheb }}\right)$ is simply given by $\gamma_{\text {cheb }}(t)=2 \cos (2 \pi t)$, hence $\gamma_{\text {cheb }}(t)=\gamma_{\text {cheb }}(s)$ if and only if $t=-s$.

We would like to show that $G$ is the mating of $f_{\theta}$ with $f_{\text {cheb. }}$. Recall that $\gamma_{\theta}$ is the Carathéodory loop of $J\left(f_{\theta}\right)$ and $\varphi_{\theta}: K\left(f_{\theta}\right) \rightarrow \widehat{\mathbb{C}}$ is the semiconjugacy between $f_{\theta}$ and $F$ given by Theorem 7.11 Denote by $\varphi_{1}$ the composition $\pi \circ \varphi_{\theta}: K\left(f_{\theta}\right) \rightarrow \widehat{\mathbb{C}}$, which conjugates $f_{\theta}$ to the quadratic rational map $G$. It is clear from the symmetry of the construction that

$$
\varphi_{\theta}\left(\gamma_{\theta}(-t)\right)=\mathcal{I}\left(\varphi_{\theta}\left(\gamma_{\theta}(t)\right)\right)
$$

for all $t \in \mathbb{T}$. It follows that the composition $\varphi_{\theta} \circ \gamma_{\theta}$ conjugates the map $t \mapsto-t$ on $\mathbb{T}$ to the involution $\mathcal{I}$. Hence it descends to a map $\varphi_{2}: K\left(f_{\text {cheb }}\right) \rightarrow \widehat{\mathbb{C}}$ which conjugates $f_{\text {cheb }}$ to $G$. It is easy to check that the pair $\left(\varphi_{1}, \varphi_{2}\right)$ satisfies the conditions of Definition IIa of the introduction. Hence,

Theorem 8.5 (Mating with the Chebyshev map). Let $0<\theta<1$ be any irrational of bounded type. Then there exists a quadratic rational map $G$ such that

$$
G=f_{\theta} \sqcup f_{\text {cheb }} .
$$

Moreover, $G$ is unique up to conjugation with a Möbius transformation. 


\section{REFERENCES}

[AB] L. Ahlfors and L. Bers, Riemann's mapping theorem for variable metrics, Annals of Math., 72 (1960) 385-404. MR 22:5813

[A] P. Atela, Bifurcations of dynamic rays in complex polynomials of degree two, Erg. Th. and Dyn. Sys., 12 (1991) 401-423. MR 94d:58128

[BS] S. Bullett and P. Sentenac, Ordered orbits of the shift, square roots, and the devil's staircase, Math. Proc. Camb. Phil. Soc., 115 (1994) 451-481. MR 95j:58043

[dFdM] E. de Faria and W. de Melo, Rigidity of critical circle mappings I, J. Eur. Math. Soc. (JEMS) 1 (1999), no. 4, 339-392. CMP 2000:05

[Do1] A. Douady, Algorithms for computing angles in the Mandelbrot set, in "Chaotic Dynamics and Fractals," ed. Barnsley and Demko, Academic Press (1986) 155-168. CMP 19:01

[Do2] A. Douady, Systèmes dynamiques holomorphes, Seminar Bourbaki, Astérisque, 105-106 (1983) 39-63. MR 85h:58090

[Do3] A. Douady, Disques de Siegel et anneaux de Herman, Seminar Bourbaki, Astérisque, 152-153 (1987) 151-172. MR 89g:30049

[DE] A. Douady and C. Earle, Conformally natural extension of homeomorphisms of the circle, Acta Math., 157 (1986) 23-48. MR 87j:30041

[DH] A. Douady and J. Hubbard, A proof of Thurston's topological characterization of rational functions, Acta Math., 171 (1993) 263-297. MR 94j:58143

[Ep] A. Epstein, Counterexamples to the quadratic mating conjecture, Manuscript in preparation.

[Ha] P. Haïssinsky, Chirurgie parabolique, C. R. Acad. Sci. Paris, 327 (1998) 195-198. MR 99i:58127

[He] M. Herman, Conjugaison quasisymetrique des homeomorphismes analytique des cercle a des rotations, Manuscript.

[Luo] Jiaqi Luo, Combinatorics and holomorphic dynamics: Captures, matings, Newton's method, Thesis, Cornell University, 1995.

[Lyu] M.Yu. Lyubich, The dynamics of rational transforms: The topological picture, Russian Math. Surveys 41 (1986) 43-117.

[Mc] C. McMullen, Complex Dynamics and Renormalization, Annals of Math. Studies, vol. 135, 1994. MR 96b:58097

[dMvS] W. de Melo, S. van Strien, One-dimensional dynamics, Springer-Verlag, 1993. MR 95a:58035

[Mi1] J. Milnor, Dynamics in One Complex Variable: Introductory Lectures, Vieweg, 1999 (Available from the American Mathematical Society). CMP 2000:03

[Mi2] J. Milnor, Geometry and dynamics of quadratic rational maps, Experiment. Math., 2 (1993) 37-83. MR 96h:58094

[Mi3] J. Milnor, Periodic orbits, external rays, and the Mandelbrot set: An expository account, Asterisque 261 (2000). CMP 2000:12

[Mi4] J. Milnor, Pasting together Julia sets - a worked out example of mating, to appear.

[Mo] R.L. Moore, Concerning upper semi-continuous collection of continua, Trans. Amer. Math. Soc., 27 (1925) 416-428. CMP 95:18

[Pe] C. Petersen, Local connectivity of some Julia sets containing a circle with an irrational rotation, Acta Math., 177 (1996) 163-224. MR 98h:58164

[Re1] M. Rees, Realization of matings of polynomials as rational maps of degree two, Manuscript, 1986.

[Re2] M. Rees, A partial description of parameter space of rational maps of degree two: part I, Acta Math., 168 (1992) 11-87. MR 93f:58205

[Sh] M. Shishikura, On a theorem of M. Rees for matings of polynomials, London Math. Soc. Lecture Note Ser., 274. CMP 2000:14

[Si] C. L. Siegel, Iteration of analytic functions, Ann. of Math., 43 (1942) 607-612. MR 4:76C

[ST] M. Shishikura and L. Tan, A family of cubic rational maps and matings of cubic polynomials, Experiment. Math., 9 (2000) 29-53. CMP 2000:12

[Sw] G. Swiatek, Rational rotation numbers for maps of the circle, Comm. Math. Phys., 119 (1988) 109-128. MR 90h:58077

[Tan] L. Tan, Matings of quadratic polynomials, Erg. Th. and Dyn. Sys. 12 (1992) 589-620. MR 93h:58129 
[TY] L. Tan and Y. Yin, Local connectivity of the Julia set for geometrically finite rational maps, Sci. China Ser. A 39 (1996) 39-47. MR 97g:58142

[Ya] M. Yampolsky, Complex bounds for renormalization of critical circle maps, Erg. Th. and Dyn. Sys., 19 (1999) 227-257. MR 2000d:37053

[Yo1] J.C. Yoccoz, Il n'y a pas de contre-example de Denjoy analytique, C. R. Acad. Sci. Paris Ser. I Math., 298 (1984) 141-144. MR 85j:58134

[Yo2] J.C. Yoccoz, Petits Diviseurs en Dimension 1, Astérisque 231, 1995.

[Za1] S. Zakeri, Biaccessibility in quadratic Julia sets I-II, to appear in Erg. Th. and Dyn. Sys.

[Za2] S. Zakeri, Dynamics of cubic Siegel polynomials, Comm. Math. Phys., 206 (1999) 185-233. CMP 2000:07

Institut des Hautes Études Scientifiques, 35 route de Chartres, F-91440, Bures-SurYvette, France

E-mail address: yampol@ihes.fr

Current address: Department of Mathematics, University of Toronto, Toronto, Ontario, Canada M5S 3G3

E-mail address: yampol@math.toronto.edu

Department of Mathematics, University of Pennsylvania, Philadelphia, Pennsylvania 19104-6395

E-mail address: zakeri@math.upenn.edu 